\title{
Malachite green "a cationic dye" and its removal from aqueous solution by adsorption
}

\author{
Nirav P. Raval ${ }^{1} \cdot$ Prapti U. Shah $^{1} \cdot$ Nisha K. Shah ${ }^{2}$
}

Received: 12 October 2016/Accepted: 28 November 2016/Published online: 18 December 2016

(C) The Author(s) 2016. This article is published with open access at Springerlink.com

\begin{abstract}
Adsorption can be efficiently employed for the removal of various toxic dyes from water and wastewater. In this article, the authors reviewed variety of adsorbents used by various researchers for the removal of malachite green $(\mathrm{MG})$ dye from an aqueous environment. The main motto of this review article was to assemble the scattered available information of adsorbents used for the removal of MG to enlighten their wide potential. In addition to this, various optimal experimental conditions (solution $\mathrm{pH}$, equilibrium contact time, amount of adsorbent and temperature) as well as adsorption isotherms, kinetics and thermodynamics data of different adsorbents towards MG were also analyzed and tabulated. Finally, it was concluded that the agricultural solid wastes and biosorbents such as biopolymers and biomass adsorbents have demonstrated outstanding adsorption capabilities for removal of MG dye.
\end{abstract}

Keywords Malachite green · Wastewater - Biosorbents · Biopolymers · Nanomaterials

Electronic supplementary material The online version of this article (doi:10.1007/s13201-016-0512-2) contains supplementary material, which is available to authorized users.

Nirav P. Raval

nirav.raval90@gmail.com

Prapti U. Shah

prapti.shah1126@gmail.com

Nisha K. Shah

nish_chem2004@yahoo.com

1 Department of Environmental Science, School of Sciences, Gujarat University, Ahmedabad 380 009, Gujarat, India

2 Department of Chemistry, School of Sciences, Gujarat University, Ahmedabad 380 009, Gujarat, India

\section{Introduction}

Till the late nineteenth century, all the colorants were extracted from shells, flowers, roots, insects and molluscs. But, with the historic discovery of first synthetic dye, Mauveine, most of the uses of natural dyes have been replaced with synthetic ones as they can be manufactured on a large scale (Gordon and Gregory 1987). At present, more than 1,00,000 synthetic dyes exist with an annual production of over $7 \times 10^{5}$ tonnes/year and are mostly associated with water pollution (Mittal et al. 2009; Gupta et al. 2011; Saravanan et al. 2016).

Malachite green (MG) is water soluble cationic dye that appears as green crystalline powder and belongs to triphenylmethane category (Raval et al. 2016b). The important physicochemical characteristics of MG were presented in Tables 1 and Table 2 narrated its applications and toxic effects. It was revealed from the Table 2 that the extensive usage of MG dye has caused several health hazards and hence, proper treatment of effluent containing MG dye is extremely necessary.

Many treatment technologies have been applied to decolorize MG from aqueous medium, such as biodegradation and decolorization (An et al. 2002; Levin et al. 2004; Ren et al. 2006; Eichlerová et al. 2006; Daneshvar et al. 2007a, b; Ayed et al. 2008; Ali et al. 2009; Wu et al. 2009; Cheriaa and Bakhrouf 2009; Ayed et al. 2010; Du et al. 2011; Khataee et al. 2011a, b; Shedbalkar and Jadhav 2011; Hu et al. 2011; Abd-El-Kareem and Taha 2012); photocatalytic degradation (Hasnat et al. 2003; Kominami et al. 2003; Lv et al. 2005; Yang et al. 2005; Chen et al. 2007; Saylkan et al. 2007a, b, 2008; Tayade et al. 2007; Wang et al. 2008a; Ju et al. 2008; Asiltürk et al. 2009; Prado and Costa 2009; Bansal et al. 2009; Kaneva et al. 2010; Liu et al. 2010; Bojinova and Dushkin 2011; Chen 
et al. 2011; Tolia et al. 2012; Saha et al. 2012; Aliyan et al. 2013); photooxidative degradation (Modirshahla and Behnajady 2006); Solar degradation (Pirsaheb et al. 2015); coagulation-flocculation (Oladoja and Aliu 2009; Man et al. 2012); ozonation (Zhou et al. 2013); fenton reagent (Chen et al. 2002; Karimi et al. 2012; Bai et al. 2013; Nidheesh et al. 2013); Solvent extraction (Pandit and Basu 2004); sonochemical and sonophotocatalytic degradation (Berberidou et al. 2007; Bejarano-Pérez and Suárez-Herrera 2008; Moumeni and Hamdaoui 2012). However, all these techniques have certain limitations in terms of design, dye separation efficiency, cost and effectiveness. Advantages and disadvantages of various techniques used for the removal of $\mathrm{MG}$ dye have been summarized in Table 3 (Dawood and Sen 2014; Yang et al. 2014). More than 250 research articles have been published related to the adsorption of MG which confirmed that amongst all the available treatment techniques, adsorption has more frequently used one.

In addition, though there was a number of review articles such as Microbial decolorization of textile-dye containing effluents: a review by Banat et al. (1996); Fungal decolorization of dye wastewaters: a review by $\mathrm{Fu}$ and Viraraghavan (2001); Remediation of dyes in textile effluent: a critical review on current treatment technologies with a proposed alternative Robinson et al. (2001); The removal of color from textile wastewater using whole bacterial cells: a review by Pearce et al. (2003); Removal of synthetic dyes from wastewaters: a review by Forgacs et al. (2004); Decolourization of industrial effluents-available methods and emerging technologies - a review by Anjaneyulu et al. (2005); Adsorption-desorption characteristics of phenol and reactive dyes from aqueous solution on mesoporous activated carbon prepared from waste tires by Tanthapanichakoon et al. (2005); Non-conventional low-cost adsorbents for dye removal: a review by Crini (2006); Methods of dye removal from dye house effluent-an overview by Mondal (2008); Agricultural based activated carbons for the removal of dyes from aqueous solutions: a review by Demirbas (2009); Application of low-cost adsorbents for dye removal - a review by Gupta and Suhas (2009); Biodegradation of synthetic dyes-a review by Ali (2010); Decolorization of dye wastewaters by biosorbents: A review by Srinivasan and Viraraghavan (2010); Cationic and anionic dye adsorption by agricultural solid wastes: a comprehensive review by Salleh et al. (2011); A review on applicability of naturally available adsorbents for the removal of hazardous dyes from aqueous waste by Sharma et al. (2011); Adsorption of dyes and heavy metal ions by chitosan composites: a review by Wan Ngah et al. (2011); Microbial decolouration of azo dyes: A review by Solís et al. (2012); Adsorption of dyes using different types of sand: a review by Bello et al. (2013); Adsorptive removal of dyes from aqueous solution onto carbon nanotubes: a review by Gupta et al. (2013); Agricultural peels for dye adsorption: a review of recent literature by Anastopoulos and Kyzas (2014); Magnetic composite an environmental super adsorbent for dye sequestration-a review by Sivashankar et al. (2014); Dye and its removal from aqueous solution by adsorption: a review by Yagub et al.

Table 3 Advantages and disadvantages of various methods used for the removal of malachite green dye (Dawood and Sen 2014; Yagub et al. 2014)

\begin{tabular}{|c|c|c|}
\hline Techniques & Advantages & Disadvantages \\
\hline \multicolumn{3}{|l|}{ Chemical methods } \\
\hline $\begin{array}{l}\text { Coagulation- } \\
\text { flocculation }\end{array}$ & $\begin{array}{l}\text { Simple and economically feasible. Short detention time and } \\
\text { low capital costs. Good removal efficiencies }\end{array}$ & $\begin{array}{l}\text { High sludge production. Handling and disposal problems. } \\
\text { High cost of chemicals for pH adjustment. Dewatering and } \\
\text { sludge handling problems }\end{array}$ \\
\hline Ozonation & $\begin{array}{l}\text { Ozone can be applied in its gaseous state and does not } \\
\text { increase the volume of wastewater and sludge }\end{array}$ & Short half-life (20 min) \\
\hline $\begin{array}{c}\text { Fenton's } \\
\text { reagent }\end{array}$ & $\begin{array}{l}\text { Capable of decolorizing wide variety of wastes. No attention } \\
\text { in volume. Effective decolorization of both soluble and } \\
\text { insoluble dyes }\end{array}$ & Prohibitively expensive. Sludge generation \\
\hline \multicolumn{3}{|l|}{ Biological methods } \\
\hline Biodegradation & Economically attractive. Publicly acceptable treatment & $\begin{array}{l}\text { Slow process. Necessary to create an optimal favorable } \\
\text { environment. Maintenance and nutrition requirements }\end{array}$ \\
\hline \multicolumn{3}{|c|}{ Emerging technologies } \\
\hline Photocatalysis & $\begin{array}{l}\text { Process carried out at ambient conditions. Inputs are atoxic } \\
\text { and inexpensive. Complete mineralization with shorter } \\
\text { detention times. No sludge production }\end{array}$ & $\begin{array}{l}\text { Effective for small amount of colorants. Expensive process. } \\
\text { Formation of by-products }\end{array}$ \\
\hline Sonication & Simplicity in use. Very effective in integrated systems & Relatively new method and awaiting full scale application \\
\hline
\end{tabular}


(2014), none of them has given comprehensive knowledge on the adsorptive removal of MG from aqueous media.

Therefore, in this review article we tried to congregate the available information on various adsorbents used for the removal of $\mathrm{MG}$, a cationic dye, from water and wastewater. The main objective of this review article was to compile an extensive list of adsorbents which will help the future generation to furnish comprehensive up-to-date research summary on adsorbents used for removal of MG dye. Also the reported optimal experimental conditions (solution $\mathrm{pH}$, contact time and amount of adsorbent) as well as adsorption isotherms, kinetics and thermodynamics data of different adsorbents towards MG were also mentioned. The authors embolden the readers to refer to the original research articles for information regarding the experimental conditions.

\section{Dyes and their classification}

Dyes (a natural or synthetic substance) are mainly chemical compounds that can affix themselves on the surface of fabrics to impart color (Yagub et al. 2014). It refers as a substance, usually organic, which is designed to be absorbed or adsorbed by, made to react with, or deposited within a substrate to impart color to that substrate with some degree of permanence (Burkinshaw 2016). Dyes may be classified in several ways (Adegoke and Bello 2015). Figure 1 summarizes the complete classification of dyes.

\section{Based on the source of materials: Dyes can be classified mainly into two types based on their origin}

\section{Natural dyes}

Natural dyes can be derived from the three primary sources_-plants, animals and minerals. They are mostly negatively charged. Positively charged natural dyes can also exist, but they are not very common. Irrespective of the source of origin, natural dyes can be categorized as:

1. Substantive dyes: Those dyes which become chemically fixed to the fiber without the aid of any other chemicals or additives are referred to as substantive dyes or direct dyes.

2. Adjective dyes: Those natural dyes which require an added substance or mordant to make the dyes colorfast are known as adjective dyes or mordant dyes. Most of the natural dyes belong to this category (Cardon 2007).

\section{Synthetic dyes}

Dyes derived from organic or inorganic compounds are known as synthetic dyes. Because of their easy use, cost effectiveness and wide range of colors these dyes became popular.

\section{Based on their method of application to the substrate}

Acid dyes

Acid dyes are highly water soluble anionic dyes which contain one or more sulfonic groups/substituents and other acidic groups. They have better light fastness compare to basic dyes. The existence of sulfonic acid groups upsurge their solubility in water and give the dye molecules a negative charge. Acid dyes can be further divided into two sub-groups:

1. Acid-leveling dyes: These dyes are smaller or medium sized, and show a moderate affinity for wool fibers. Hence, due to average affinity these dyes are not very resistant to washing.

2. Acid-milling dyes: These dyes are larger than acidleveling dyes, and show a much stronger intermolecular attractions for wool fibers. Due to this strong affinity these dyes are very resistant to washing.

\section{Basic dyes}

These water soluble dyes possess cationic functional groups such as $-\mathrm{NR}^{3+}$ or $=\mathrm{NR}^{2+}$. They are generally amino and substituted amino compounds soluble in acid. They become attached to the fibers by formation of ionic bonds with fiber's anionic groups.

\section{Direct dyes}

Although these dyes are water soluble anionic dyes, they cannot be classified as acid dyes because the acid groups are not the means of attachment to the fiber. Since, these dyes do not require any kind of fixing, they are called direct dyes.

\section{Mordant dyes}

A latin word mordant means 'to bite'. It acts as 'fixing agent' to improve the color fastness of some acid dyes, because they have the ability to form insoluble colored complexes with metal ions. They are usually metal salts. Besides, alum there is a large range of other metallic salt mordants available. Aluminum, chromium and iron salts are frequently used for synthetic mordant dyes.

\section{Reactive dyes}

Fiber reactive dyes will form a covalent bond while they react with the cellulosic fiber. As a result they are very 


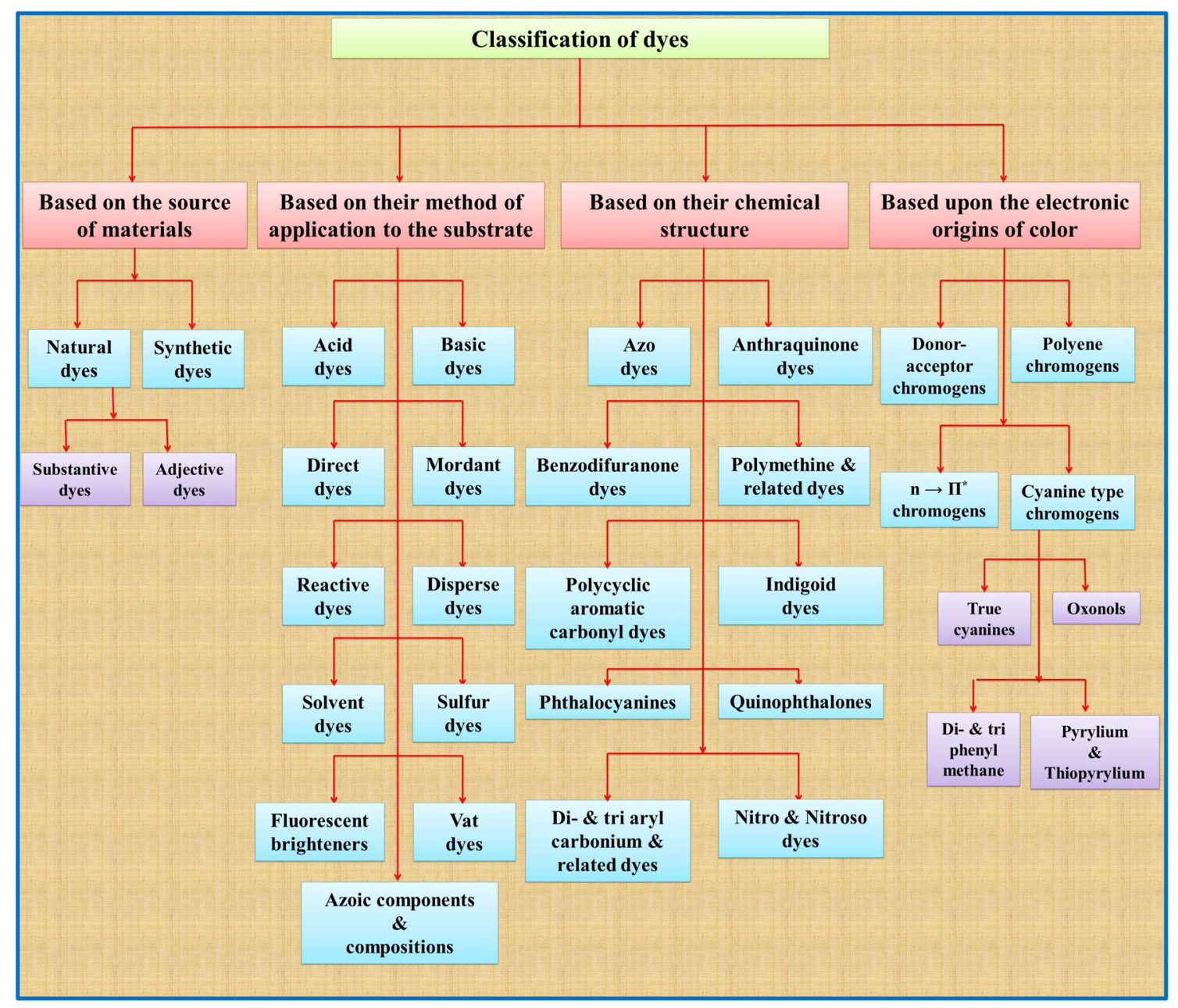

Fig. 1 Broad classification of dyes

difficult to remove, once attached with the fiber. Initially, these dyes were designed for cellulose fibers but now some fiber-reactive dyes for protein and polyamide fibers are also commercially available.

\section{Disperse dyes}

These dyes have substantially low solubility in water. Their structure is small, planner and non-ionic with attached polar functional groups, such as $-\mathrm{NO}_{2}$ and $-\mathrm{CN}$. They are mainly used for the dyeing of polyesters because they can interact with the polyester chains by forming dispersed particles.

\section{Solvent dyes}

These dyes are insoluble in water but soluble in alcohols, chlorinated hydrocarbons and liquid ammonia. They are mainly used for coloring plastics, synthetic fibers, gasoline, oils and waxes.

\section{Sulfur dyes}

Sulfur dyes are applied to cotton from an alkaline reducing bath with sodium sulfide as the reducing agent. These dyes are water insoluble but they are soluble in their reduced form and exhibit affinity for cellulose. They are low cost and have good fastness to light, washings and acids. The actual structures of sulfur dyes are largely unknown although it is considered that they possess sulfur-containing heterocyclic rings.

\section{Based on their chemical structure: (Gordon and Gregory 1987; Waring and Hallas 1990)}

Azo dyes

Approximately half of all dyes are azo dyes, making them the largest group of synthetic colorants used in textile industries. This type of dyes contain minimum one azo group but can also contain two or three, but very rarely, 
four azo groups. Azo dyes are complex aromatic compounds with significant structural diversity and are of great environmental concern because the reductive cleavage of azo linkages is responsible for the formation of amines, which are classified as toxic and carcinogenic (Raval et al. 2016a).

\section{Anthraquinone dyes}

Anthraquinone dyes are the second most important class after azo dyes. They are also one of the oldest types of dyes since they have been found in the covering of mummies dating back over 4000 years. In contrast to the azo dyes, which have no natural counterparts, all the natural red dyes were anthraquinones. The lower tinctorial strength and reduced flexibility increase the production cost of anthraquinone dyes and hence, they are not as widely used as azo dyes.

\section{Benzodifuranone dyes}

Benzodifuranone (BDF) dyes are one such type which attacking anthraquinone dyes. The BDF chromogen is one of the very few novel chromogens to have been accidently discovered this century

\section{Polymethine and related dyes}

Dyes containing a conjugated chain of carbon atoms terminated by an ammonium group and, in addition, a nitrogen, sulfur, or oxygen atom, or an equivalent unsaturated group is termed as polymethine dyes. This type of dyes may be neutral, cationic or anionic. The best known cationic polymethine dyes are the cyanine dyes. Cyanine dyes cannot be used as textile dyes because they have poor light fastness. They are used in photography.

\section{Polycyclic aromatic carbonyl dyes}

Polycyclic aromatic carbonyl dyes have one or more carbonyl groups. These carbonyl groups are linked by a quinonoid system. These dyes are relatively very large molecules which are made up from smaller units of anthraquinones. These dyes are often termed as anthraquinonoid vat dyes because they are applied to the substrate by a vatting process.

\section{Indigoid dyes}

Indigoid dyes also contain carbonyl groups, similar to the anthraquinone, benzodifuranone and polycyclic aromatic carbonyl dyes. They are vat dyes and represent one of the oldest known classes of dyes.

\section{Phthalocyanines}

Phthalocyanines are one of the novel chromophores which have been discovered since the nineteenth century. They are structurally similar to the natural porphyrin pigments haemin and chlorophyll. These natural pigments have poor stability, but phthalocyanines have exceptional stability and tinctorially stronger. All the phthalocyanine compounds are blue to green in color because substituents in their fused benzene rings exert only a minor sway on the color of phthalocyanines. They are widely used in printing inks and paints.

\section{Di- and tri-aryl carbonium and related dyes}

Di- and triarylcarbonium dyes and their heterocyclic derivatives comprise the oldest class of synthetic dyes-the majority of which were discovered in the 19th and early 20th century. Diphenylmethane and Triphenylmethane dyes are the most important aryl carbonium dyes. Structurally and property wise they are similar to the cyanine dyes i.e. exceptional brightness, high tinctorial strength and low light fastness.

\section{Nitro and nitroso dyes}

Nitro and nitroso dyes are minor commercial importance and constitute only a fraction of the total dyes market. Nitro dyes have simple chemical structures. They are small aromatic molecules which contain minimum one nitro group and one or more amino or hydroxy groups. Initially, the nitro dyes were acid dyes which were used for dyeing the natural animal fibers i.e. wool and silk. Picric acid was the first synthetic nitro dye which imparted a greenishyellow color to silk but could not be used for longer period of time because of its toxicity and poor fastness properties.

Nitroso dyes are prepared by nitrosation of the appropriate phenol or naphthol. They are generally metal complex derivatives of $o$-nitrosophenols or naphthols. Nitroso dyes are polygenetic because they form differently colored complexes with different metals. They have good light fastness.

\section{Based upon the electronic origins of color}

Griffith (1976, 1981) has classified dyes based on the electronic mechanism by which color is generated. According to this, purely on theoretical basis, dyes can be arranged into four classes.

(II) Donor-acceptor chromogens

(III) Polyene chromogens

(IIII) $\mathrm{n} \rightarrow \Pi^{2}$ chromogens 
(IIV) Cyanine type chromogens

\section{Literature on various adsorbents used for the removal of MG}

See (Fig. 2)

\section{Activated carbon adsorbents}

Activated carbons (AC) are known as very efficacious adsorbents due to their large surface area, a micro-porous structure and a high degree of surface reactivity. However, due to their high production costs, these materials tend to be more expensive than other adsorbents. Four classes of AC include: powder (PAC), granular (GAC), fibrous (ACF) and cloth (ACC). Also two more frequently used ACs are: (1) commercial activated carbons and (2) activated carbons prepared from agricultural waste materials (Raval et al. 2016c).

\section{Commercial activated carbons}

A number of commercially available activated carbons such as powdered activated carbon (Kumar 2006); coalbased activated carbon (Aitcheson et al. 2000); commercially available powdered activated carbon (CPAC) (Malik et al. 2007); activated carbon prepared from lignite (Önal et al. 2007); activated Charcoal (Iqbal and Ashiq 2007); activated carbon prepared from Tuncbileklignite (Önal et al. 2006); oxyhumolite (Young brown coal) (Janos et al. 2005); Ordered mesoporous carbons (OMCs) (Tian et al.
2011); etc. have been extensively studied for the adsorption of MG.

Mesoporous carbon was synthesized by Anbia and Ghaffari (2012) for the removal of a cationic dye malachite green (MG) from aqueous solution. They carried out study under various experimental conditions and concluded that the equilibrium was attained within $30 \mathrm{~min}$ of contact with adsorbent and more than $99 \%$ removal of MG was reached at the optimum $\mathrm{pH}$ value of 8.5 .

Aitcheson et al. (2000) used coal-based activated carbon 207EA (Sutcliffe Speakman) for the removal of aquaculture therapeutants (Malachite Green, formalin, Chloramine-T and Oxytetracycline) which were widely used in aquaculture to control fish parasites and disease. The adsorption process was well fitted to the Freundlich isotherm and confirmed that adsorption was heterogeneous in nature.

Adsorption of MG was studied by Mall et al. (2005) using commercial grade (ACC) and laboratory grade (ACL) activated carbons. Authors conducted batch adsorption studies to evaluate the effect of various parameters such as $\mathrm{pH}$, adsorbent dose, contact time and initial MG concentration on the removal of MG and concluded that the effective $\mathrm{pH}$ was 7.0 for adsorption of MG. Equilibrium reached in about $4 \mathrm{~h}$ contact time and optimum ACC and ACL dosages were 20 and $4.0 \mathrm{~g} / \mathrm{L}$, respectively.

To overcome deficiencies of CACs the use of activated carbon adsorbents derived from various agricultural waste and industrial solid waste materials have received increasing consideration in the purification of wastewater over the last several years because they are generated in excess, inexpensive, locally available and their use will

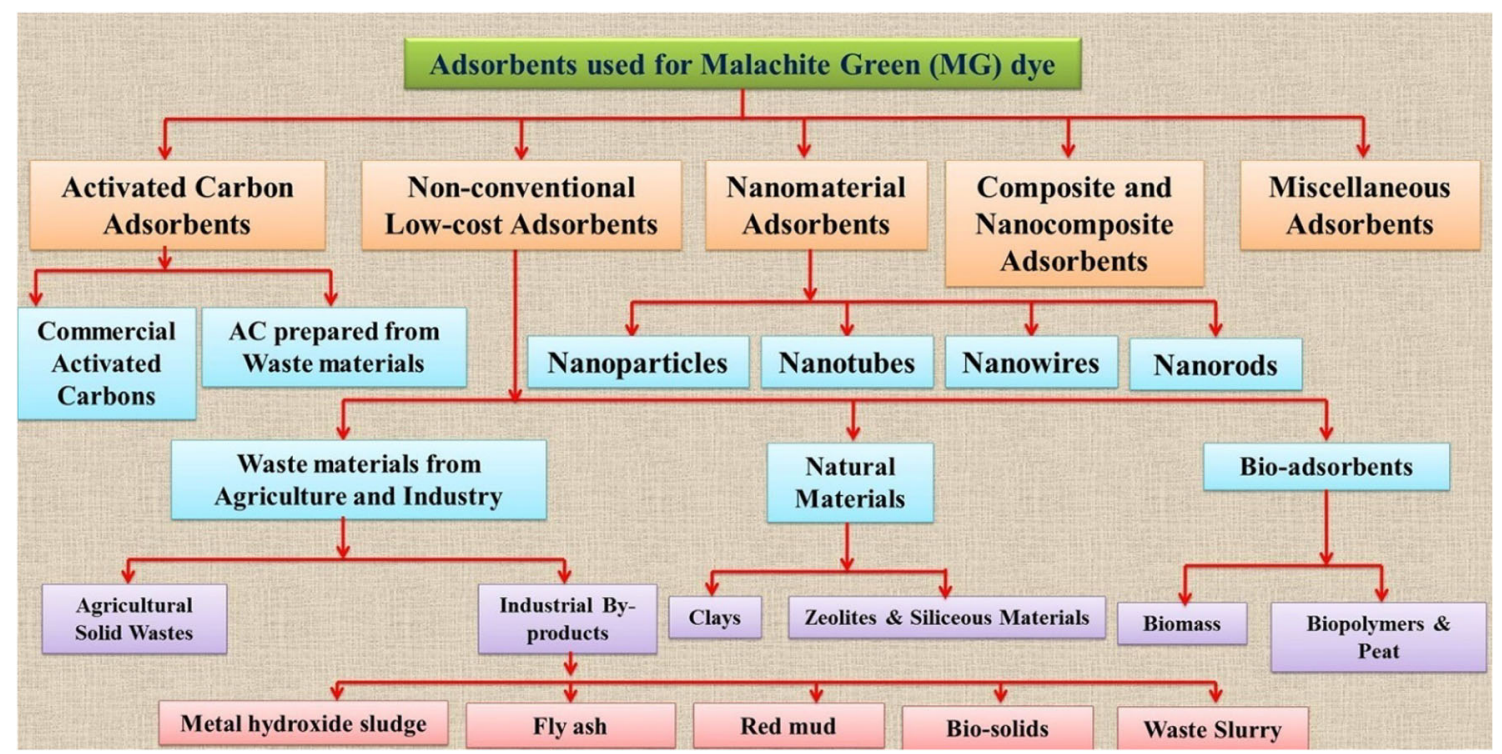

Fig. 2 Classification of adsorbents used for the removal of MG 
further solve the problem of their disposal (Pehlivan and Arslan 2007).

\section{Activated carbons prepared from agricultural and industrial solid wastes}

As biomass and other waste materials have little or no economic value and often present a disposal problem, they offer an inexpensive and additional renewable source of activated carbon. Thus, conversion of these low cost by products into activated carbon would provide an inexpensive substitute to the existing CACs, add monetary value and help in dropping the expenditure of waste disposal (Raval et al. 2016c).

Polygonum orientale Linn is an annual plant of the genus Polygonum in the family Polygonaceae, which is native to eastern Asia and widely distributed in China. This plant has a developed caudex system and generally produces large and continuous caudex masses. Therefore, this plant offers a good basis for the development of adsorbent materials. Looking upon this property of plant (Wang et al. 2010) prepared $P$. orientale Linn activated carbon (PLAC) from waste $P$. orientale Linn by chemical activation with $\mathrm{H}_{3} \mathrm{PO}_{4}$ and used as an adsorbent to remove malachite green (MG) and rhodamine B (RB) from aqueous solution.

Durian (Durio zibethinus Murray) is the most popular seasonal fruit in Southeast Asia, particularly Malaysia, Indonesia, Thailand, and the Philippines, and belongs to the family Bombacaceae. A significant percentage of the planted durian fruit crop is wasted each year. Only one third of durian fruit is edible, whereas the seeds (20-25\%) and the shells are usually discarded. The wasted durian seed represents a significant potential for the development of value-added products. Thus, Ahmad et al. (2014) studied the removal of Malachite green dye using durian seedbased activated carbon.

Rambutan (Nephelium lappaceum L.) is a popular tropical fruit which belongs to Sapindaceae family. However, due to the high consumption of rambutan's edible part, massive amount of the peel is disposed, causing a severe problem in the community as they gradually ferment and release off odors. Therefore, Ahmad and Alrozi (2011) converted rambutan peel into activated carbon by physiochemical activation for its potential to remove $\mathrm{MG}$ dye from wastewater.

Akar et al. (2013) produced active carbon from spent tea leaves (STAC) by activation with $4 \% \mathrm{NaOH}$ solution for the removal of malachite green dye because tea is basically dried and processed leaves of plant called Camellia sinensis. It is consumed by the most of people in the world and is the second most popular beverage in the world. It is estimated that between 18 and 20 billion cups of tea are drunk daily in world and disposal of spent leaves become a major problem.

Banana (Musa paradisiaca) is native to Southeast Asia, most of the bananas produced are consumed locally and $\sim 10 \%$ are exported. Massive amounts of stalk (as waste products) are disposed, resulting in a serious environmental problem through the emission of foul odors when it is left to decompose. Hence, Bello et al. (2012) attempted to use banana stalk, an abundantly available lignocellulosic agricultural waste, as a cheap and renewable precursor for activated carbon preparation for $\mathrm{MG}$ dye removal and helped in placing value on this agricultural waste and provide a potentially cheap alternative to existing commercial activated carbons.

In addition to above described materials, a wide variety of other agricultural and industrial waste materials have also been used for the preparation of activated carbon and applied for the adsorptive removal of $\mathrm{MG}$ dye from aqueous solution. It includes Tamarind fruit shells carbon (Vasu 2008); Juglans regia shells carbon (Nethaji et al. 2012); Annona squmosa seed (Santhi et al. 2010a); Groundnut shell carbon (Malik et al. 2007); Pine sawdust (Akmil-Başar et al. 2005); Tea leaves (Singh and Rastogi 2004); Bamboo (Hameed and El-Khaiary 2008a); Jute fiber (Porkodi and Vasanth Kumar 2007) and waste printed circuit boards (Kan et al. 2015), etc.

In addition, Table 4 presented various commercially available and agricultural waste based activated carbon adsorbents used for the removal of MG and their optimum conditions.

\section{Non-conventional low-cost adsorbents}

Activated carbon adsorption has been cited by the US Environmental Protection Agency as one of the best available control technologies. However, although activated carbon is a preferred sorbent, its widespread use is restricted due to high cost. To decrease the cost of treatment, attempts have been made to find inexpensive alternative adsorbents. Recently, numerous approaches have been studied for the development of cheaper and effective adsorbents. Thus, there is a need to search for new and economical adsorbent that could remove metal. In this context, agricultural by-products and industrial waste can be seen as having a great potential to be developed as a low cost sorbent. A low-cost adsorbent is defined as the one which is plentiful in nature, or is a by-product or waste from industry and requires little or no processing (Raval et al. 2016c). Literature survey reveals that multifarious materials have been utilized as adsorbents under this category. 


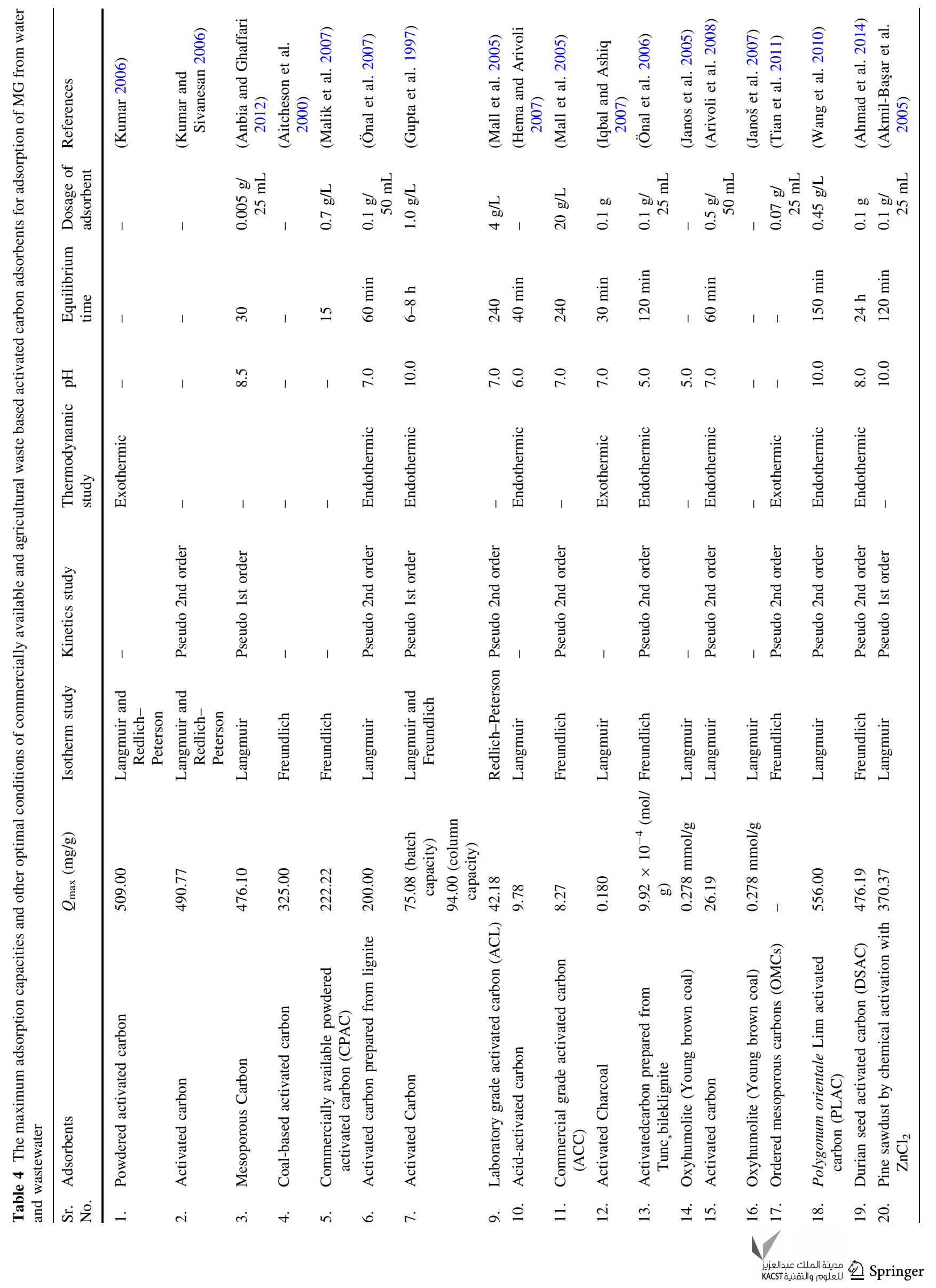




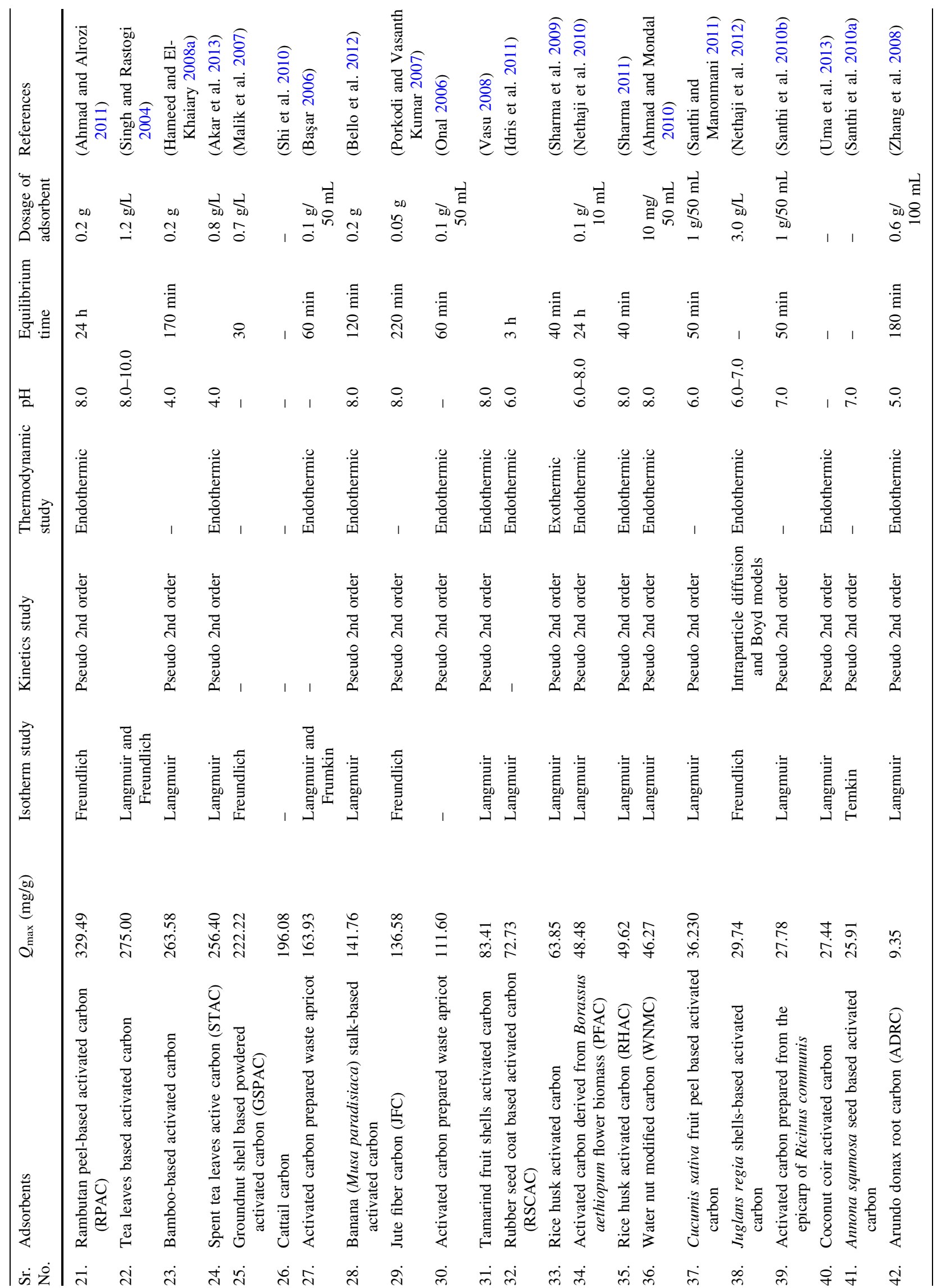




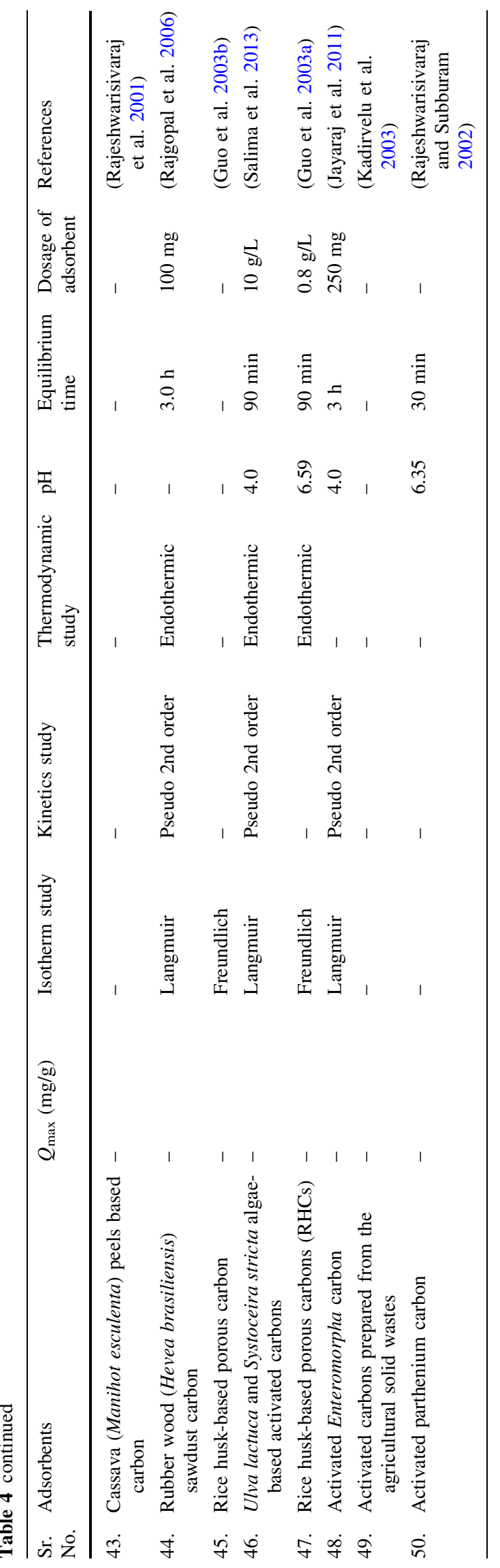

Waste materials from agriculture and industry

Agricultural solid wastes Agricultural solid wastes are relatively cheap and are available in huge quantities. They can be used as an adsorbent due to their physic-chemical properties. The utilization of these wastes can play a significant role in national economy (Crini 2006).

Orange peel is discarded in the orange-juice and softdrink industries all over the world. It has been used as an adsorbent for the removal of MG from wastewater by Kumar and Porkodi (2007). The results revealed that the adsorption process followed both Langmuir and RedlichPeterson isotherms, the adsorption capacity was $483.63 \mathrm{mg} / \mathrm{g}$.

A study of Rahman et al. (2005) reported the removal of MG from aqueous solutions onto Phosphoric acid $\left(\mathrm{H}_{3} \mathrm{PO}_{4}\right)$ and sodium hydroxide $(\mathrm{NaOH})$ treated rice husks.

Rice straw is a lignocellulosic agricultural stalk containing cellulose $(37.4 \%)$, hemi-cellulose $(44.9 \%)$, lignin (4.9\%) and silica ash (13.1\%). Gong et al. (2006) compared MG adsorption efficiency of native and thermos chemically modified rice straw and found their adsorption efficiency 94.34 and $256.41 \mathrm{mg} / \mathrm{g}$, respectively.

Chieng et al. (2015) investigated the use of breadnut (Artocarpus camansi) as a low-cost adsorbent for the removal of MG toxic dye. In Brunei Darussalam, breadnut was locally known as 'Kemangsi', whose seeds were consumed either cooked or roasted. The skin and core of Artocorpus fruits, the major parts of the fruits, were inedible and have no economical value, and discarded as waste. Authors concluded that the adsorption characteristics of breadnut skin towards MG can be significantly enhanced by modification through treatment with dilute $\mathrm{NaOH}$ solution.

Other solid wastes from cheap and readily available resources have also been successfully employed for the removal of $\mathrm{MG}$ from aqueous solution and were given in Table 5.

Industrial by-products/industrial solid wastes Industrial by products, such as metal hydroxide sludge, fly ash, red mud and bio-solids, can be used as low-cost adsorbents for MG removal. Table 6 represented the maximum adsorption capacities together with the isotherm, kinetics and thermodynamic results of various industrial solid wastes adsorbents for MG.

Fly ash: Witek-Krowiak et al. (2012) investigated the use of fly ash for the adsorption MG from solution with different contact times, initial dye concentrations, $\mathrm{pHs}$ and temperatures. The adsorption equilibrium has been described in terms of Langmuir model. The enthalpy values obtained in the experiment were not high, which suggested 
that physical sorption dominates in the process of cationic dyes bonding on microspheres of fly ash.

Bagasse fly ash (BFA) is a waste collected from the particulate separation equipment attached to the flue gas line of the sugarcane bagasse-fired boilers and has been used as an effective adsorbent for the removal of dyes. This is available in plenty, and almost free of cost from the sugar mills. In addition, bagasse fly ash generated in the sugar industry does not contain large amounts of toxic metals and hence, Mall et al. (2005) used bagasse fly ash (BFA) as adsorbent for the removal of $\mathrm{MG}$ from aqueous solutions. Maximum adsorption capacity for MG was reported as $170.33 \mathrm{mg} / \mathrm{g}$ (at $\mathrm{pH} 7.0$ and adsorbent dosage of $1.0 \mathrm{~g} / \mathrm{L}$ ) and within $240 \mathrm{~min}$ of contact time.

Waste red mud: Zhang et al. (2014b) used the acid activated (Acid treatment neutralize the alkalinity of red mud, which could also be recognized as a method for activating the material) sintering process red mud (ASRM) for the adsorption of MG. The optimum process variables were found to be $\mathrm{pH}>3.2,180 \mathrm{~min}$ of contact time and $0.25 \mathrm{~g} / 250 \mathrm{~mL}$ of adsorbent dosage. The adsorption process followed the Langmuir isotherm with $336.40 \mathrm{mg} / \mathrm{g}$ of adsorption capacity.

Activated slag: Steel plants produce granular blast furnace slag as a byproduct, and this material also causes a disposal problem. Hence, Gupta et al. (1997) utilized this waste product for the adsorption of $\mathrm{MG}$ from aqueous medium both in batch and column modes. Further, they concluded that the column capacity was found to be higher than the batch capacity. The removal takes place through a film diffusion mechanism at lower concentrations and by particle diffusion at higher concentrations.

\section{Natural materials}

Clays Clay is mineral comprised of alumina and silica that usually includes bound water. Clays have a sheet-like structure and are composed of tetrahedrally arranged silicates and octahedrally arranged aluminates. Due to their low-cost, abundance in nature, high sorption capacity, chemical and mechanical stability, layered structure and high cation-exchange capacity (CEC), clay minerals are frequently used as an adsorbent (Krauskopf 1956; da Fonseca et al. 2006). The clays always contain exchangeable ions on their surface and play vital role in the environment by acting as a natural scavengers of contaminants by taking up cations and/or anions through either ion exchange or adsorption (Babel and Kurniawan 2003).

A low-cost inorganic powder (Persian Kaolin) was used as adsorbent for the sequestration of cationic dye from aqueous solution by Tehrani-Bagha et al. (2011). The sorption of the dye malachite green on kaolin confirmed to linear form of Langmuir adsorption equation. The maximum adsorption capacity was $52.91 \mathrm{mg} / \mathrm{g}$. In addition, kinetic and thermodynamic parameters, such as enthalpy $(\Delta H)$ and free energy $(\Delta G)$ were calculated. The negative values of $\Delta \mathrm{G}$ and positive values of $\Delta H$ indicated that the dye adsorption process was spontaneous and endothermic in nature

The efficacy of montmorillonite clay had been investigated by Tahir et al. (2010) for the adsorptive removal of malachite green dye from aqueous solution. Authors concluded that the MG dye was homogenously adsorbed on the surface of montmorillonite clay adsorbent.

Bulut et al. (2008) investigated the sorption of MG from aqueous solution onto bentonite as a function of contact time, $\mathrm{pH}$ and initial dye concentration. The adsorption equilibriums have been described in terms of Langmuir, Freundlich, Temkin and Dubinin-Radushkevich models. Authors reported that the adsorption process obeyed the Langmuir isotherm and pseudo-second-order kinetic model. The results of the thermodynamic study indicated that the sorption process was endothermic and spontaneous.

Tahir and Rauf (2006) utilized the natural bentonite clay for the adsorption of MG dye from aqueous solution. The authors finally concluded that the values of sorption free energy $\left(E_{\mathrm{a}}\right)$ of $1.00-1.12 \mathrm{~kJ} / \mathrm{mol}$ indicated physical adsorption of dye on clay mineral.

Siliceous materials Siliceous rocks are sedimentary rocks that have silica $\left(\mathrm{SiO}_{2}\right)$ as the principal constituent. The most common siliceous rock is chert other types include diatomite. They commonly form silica-secreting organisms, such as radiolarians, diatoms, or some types of sponges. The natural siliceous materials used for MG adsorption were perlite, silica, glass fibers and alunite because of their high abundance, easy availability and low cost.

Govindasamy et al. (2009) studied the removal of MG from aqueous solution by perlite. Authors reported $40 \mathrm{~min}$ of contact time, $0.1 \mathrm{~g} / 50 \mathrm{~mL}$ of adsorbent dosage and 8.0-9.0 $\mathrm{pH}$ value were optimum conditions for the removal of dye. It was suggested that the adsorption of MG onto perlite was spontaneous, chemical and exothermic in nature and followed Freundlich isotherm as well as pseudo-firstorder kinetic rate model.

Silica gel is a hydrophilic porous adsorbent. Aromatic compounds are found to involve the pi-cloud in hydrogen bonding with silanol ( $\mathrm{SiOH})$ group during adsorption. Cationic and nonionic surfactants adsorb on silica surface involving hydrogen bonding (Parida et al. 2006).

Kannan et al. (2008) utilized tetrahedral silica for the adsorption of MG. The adsorbent was characterized by Fourier transformed infrared spectroscopy (FT-IR) to confirm the tetrahedral framework of silica. The 


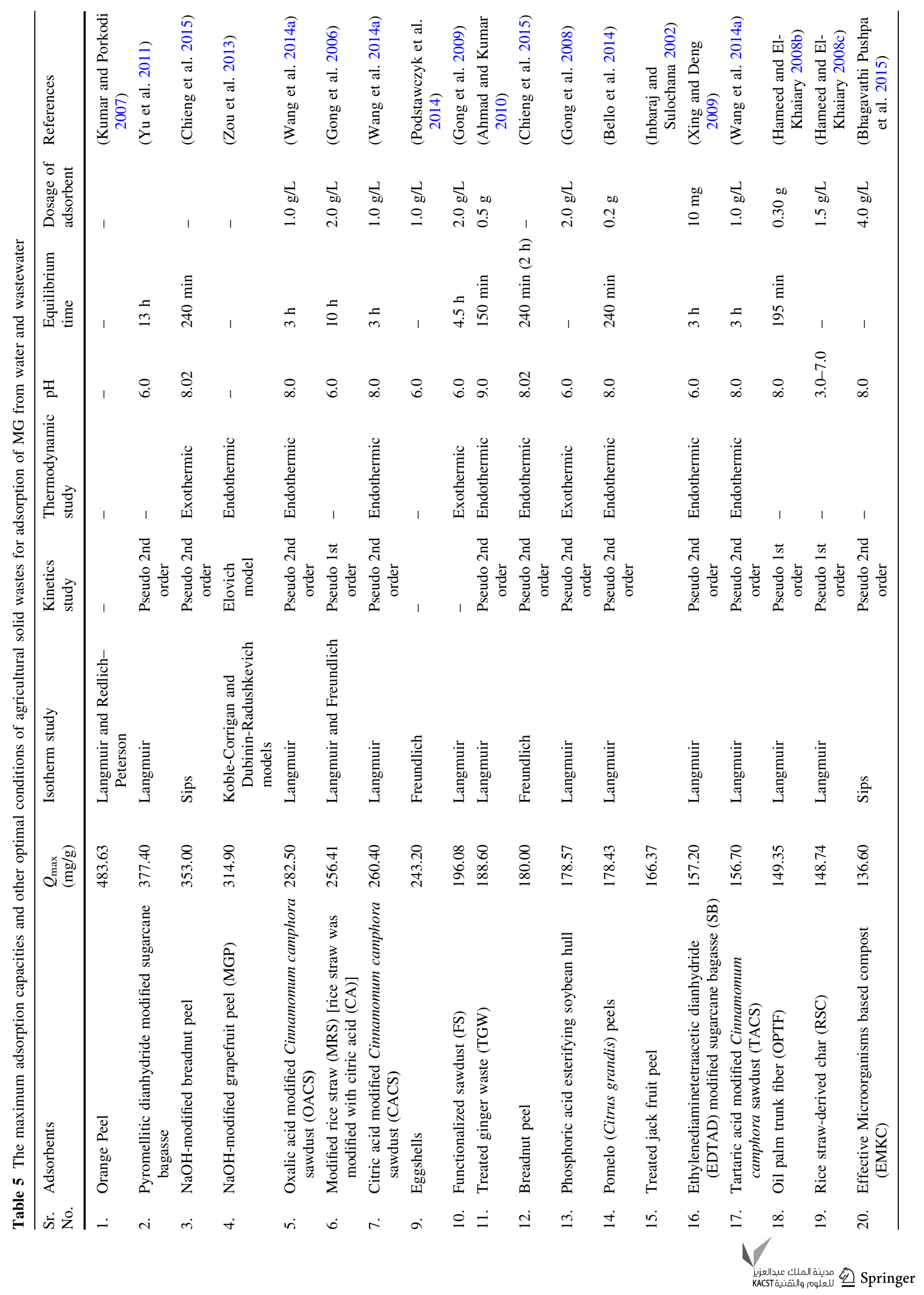




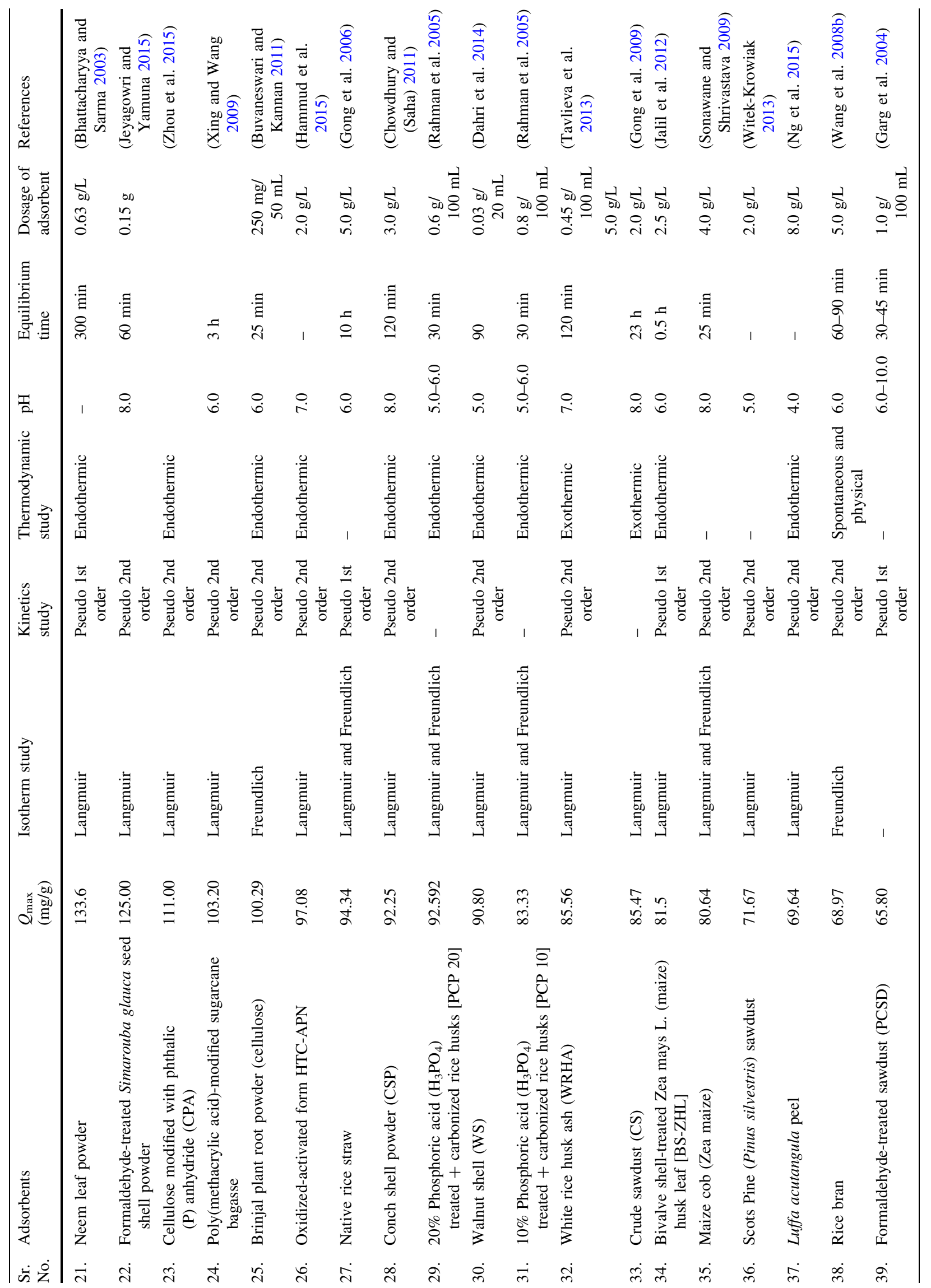




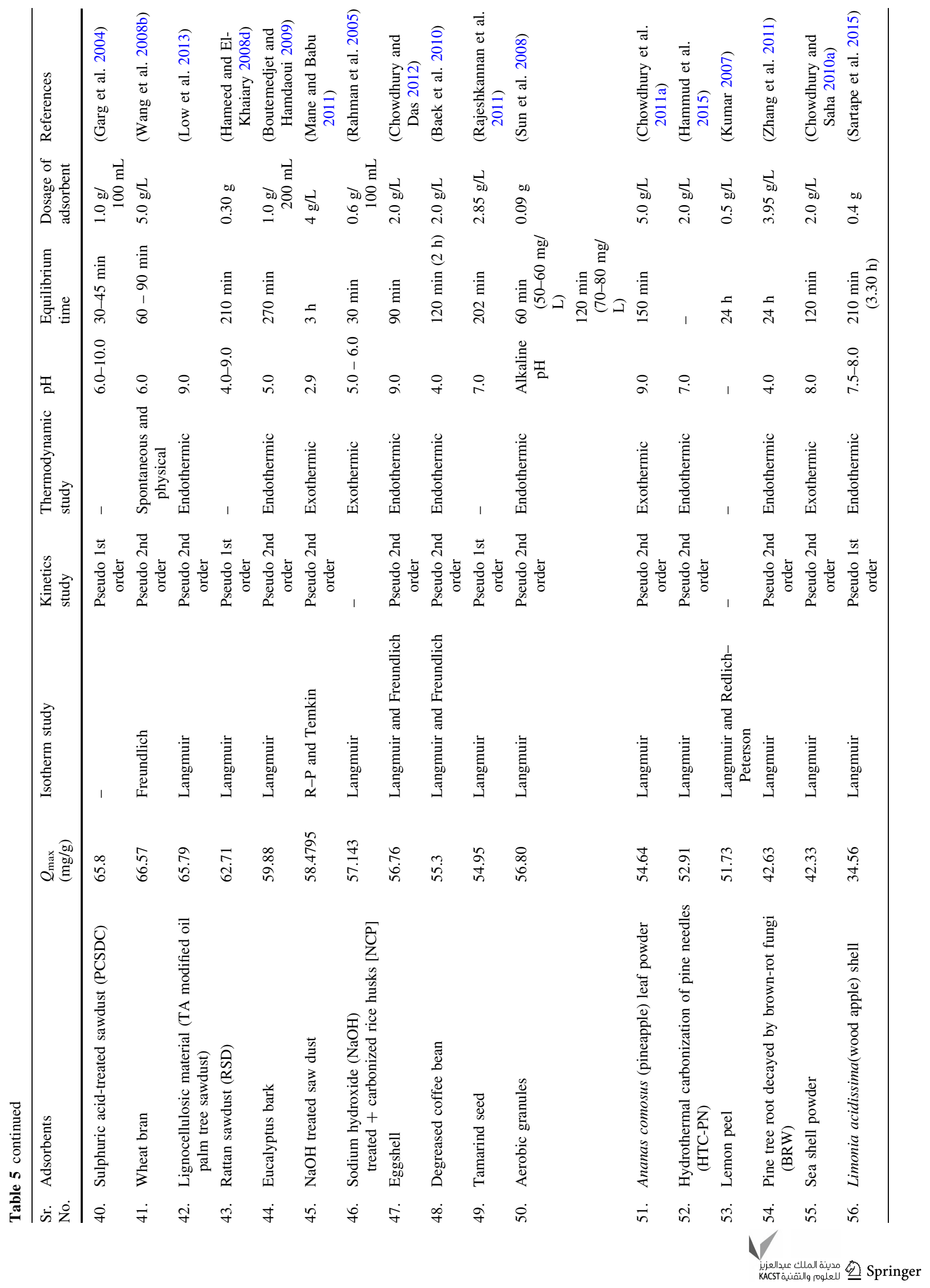




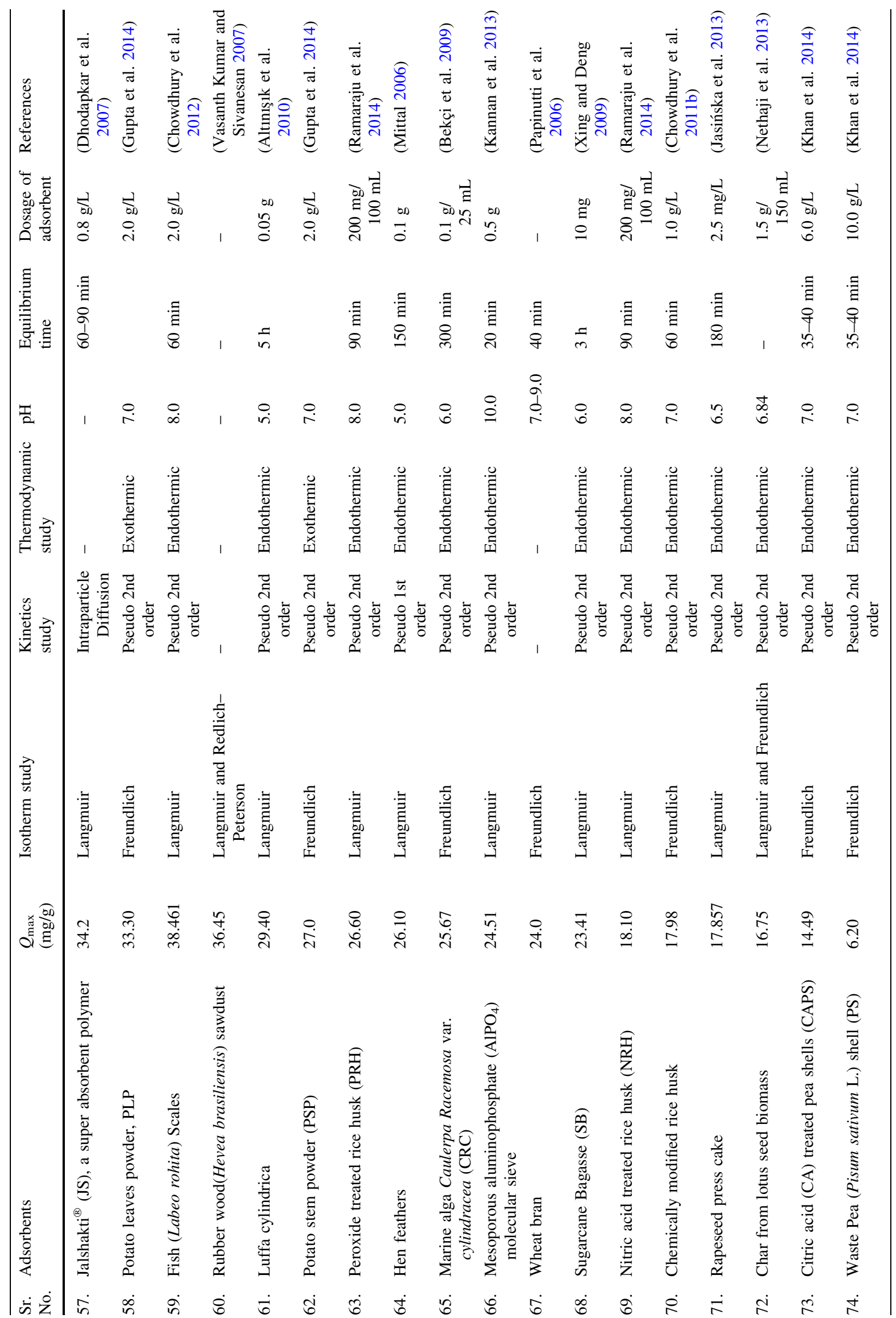




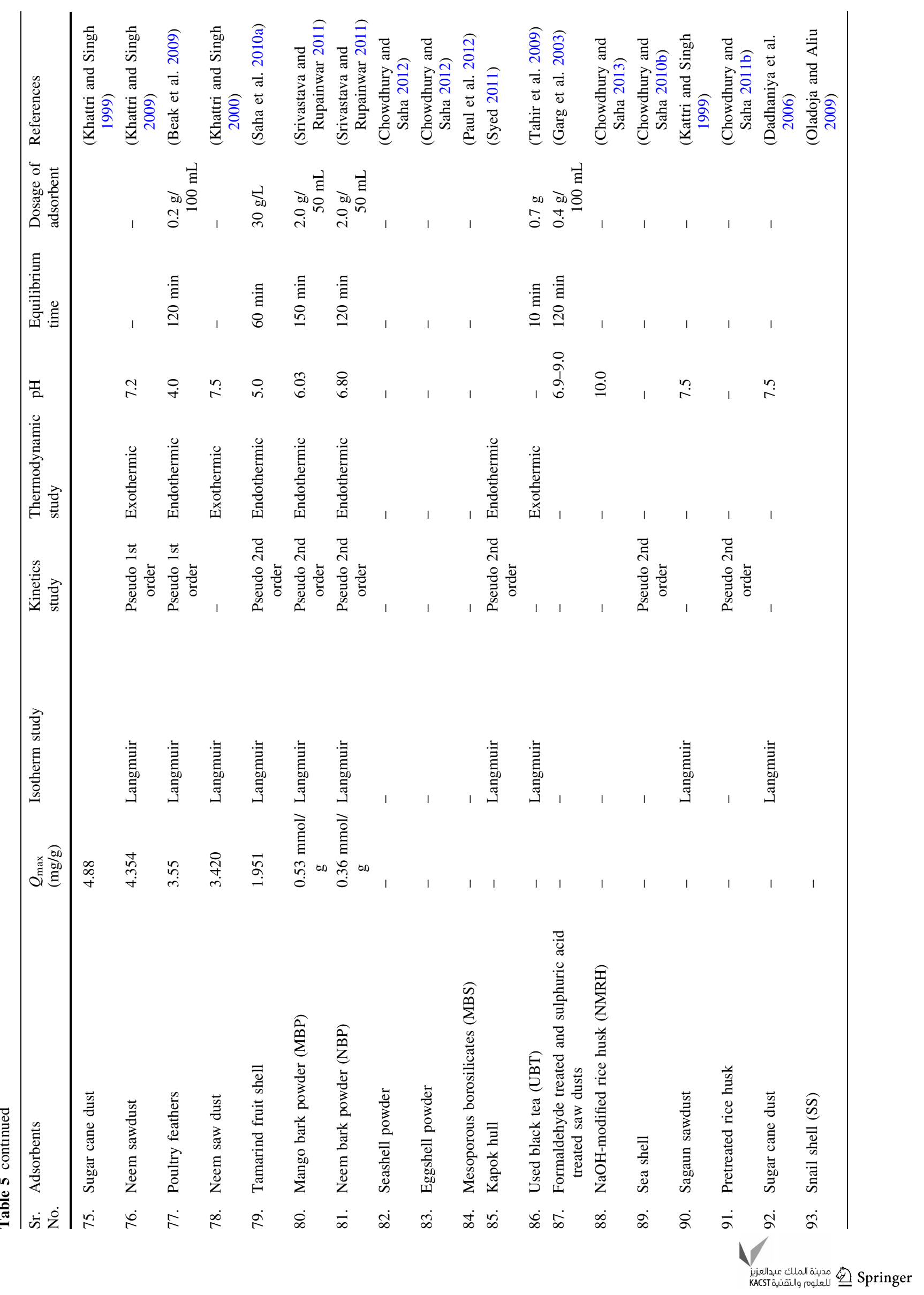




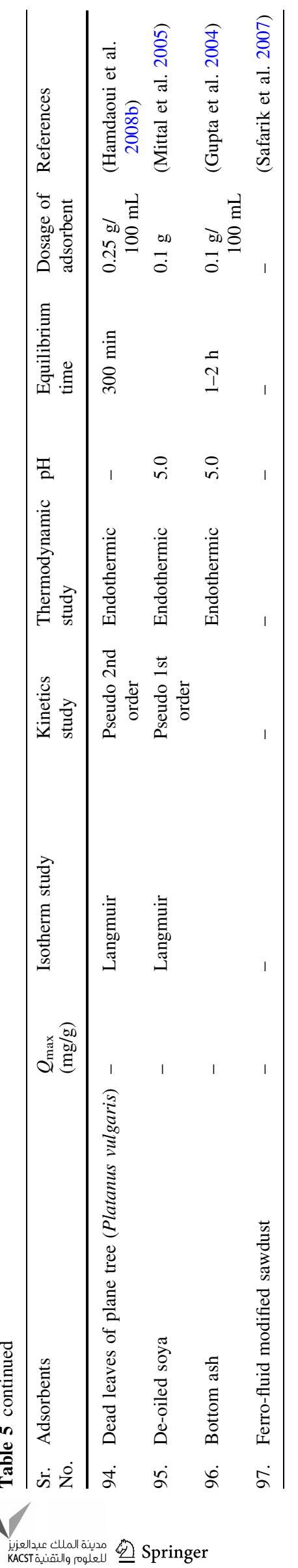

thermodynamic studies proved that the adsorption was endothermic and chemisorptions $\left(\Delta H^{0}>40 \mathrm{~kJ} / \mathrm{mol}\right)$ on silica. Recovery of dye on silica was studied from 30 to $90{ }^{\circ} \mathrm{C}$ and observed that only $3.5 \%$ was recovered from silica. The less recovery on silica proved the strong adsorption of dye on silica.

Talc is the most common silicate material, with an idealized chemical formula of $\mathrm{Mg}_{3} \mathrm{Si}_{4} \mathrm{O}_{10}(\mathrm{OH})_{2}$. Its ability to remove aqueous MG from solution had been studied by Lee et al. (2013).

Zeolites Zeolites are highly porous crystalline aluminosilicates with tetrahedrally connected three dimensional frameworks and extra-framework charge balancing cations. Zeolite structures are commonly described in terms of the size, geometry and connectivity of the pore space. The use of natural zeolite as an adsorbent has gained interest among researchers; mainly because of its sorption properties provide a combination of ion exchange and molecular sieve properties which can also be easily modified (Goursot et al. 1997; Sánchez and Pariente 2011).

Zeolites have been used as chemical sieves, as water softeners, as removers of ammonia from urban, agricultural and industrial waste waters and of toxic gases from gaseous emissions, as filters for odor control, as chemical fertilizer, herbicide and pesticide carriers and in animal nutrition (Sand and Mumpton 1978; Breck 1984; Karge and Weitkamp 1989).

Han et al. (2010) investigated the use of natural zeolite, obtained from Xinyang city in China, for the removal of MG from aqueous solution. The rate constants of sorption for different kinetic models were calculated. Good correlation coefficients were obtained for the pseudo-second order kinetic model. In addition, authors were treated spent zeolite by microwave irradiation and it was found that yield of regeneration was $85.8 \%$ in the case of microwave irradiated time $10 \mathrm{~min}$ at $160 \mathrm{~W}$.

Table 7 represented the maximum adsorption capacities together with the isotherm, kinetics and thermodynamic results of various natural materials used for the removal of MG

\section{Biosorbents}

Biosorption is defined as the accumulation and concentration of pollutants from aqueous solutions by the use of biological materials. Biological materials, such as yeast, fungi or bacterial biomass and biopolymers are used as adsorbents for the removal of MG from aqueous media (Raval et al. 2016d; Gupta et al. 2015).

Biomass Biomass is biological material derived from living, or recently living organisms, which can be applied 
Table 6 The maximum adsorption capacities and other optimal conditions of various industrial waste materials for adsorption of MG from water and wastewater

\begin{tabular}{|c|c|c|c|c|c|c|c|c|c|}
\hline $\begin{array}{l}\text { Sr. } \\
\text { No. }\end{array}$ & Adsorbents & $\begin{array}{l}Q_{\max } \\
(\mathrm{mg} / \mathrm{g})\end{array}$ & $\begin{array}{l}\text { Isotherm } \\
\text { study }\end{array}$ & $\begin{array}{l}\text { Kinetics } \\
\text { study }\end{array}$ & $\begin{array}{l}\text { Thermodynamic } \\
\text { study }\end{array}$ & $\mathrm{pH}$ & $\begin{array}{l}\text { Equilibrium } \\
\text { time }\end{array}$ & $\begin{array}{l}\text { Dosage of } \\
\text { adsorbent }\end{array}$ & References \\
\hline 1. & $\begin{array}{l}\text { Acid-activated sintering process } \\
\text { red mud (ASRM) }\end{array}$ & 336.40 & Langmuir & $\begin{array}{l}\text { Pseudo } \\
\text { 2nd } \\
\text { order }\end{array}$ & Endothermic & $>3.2$ & $180 \mathrm{~min}$ & $\begin{array}{l}0.25 \mathrm{~g} / \\
250 \mathrm{~mL}\end{array}$ & $\begin{array}{l}\text { (Zhang et al. } \\
\text { 2014b) }\end{array}$ \\
\hline 2. & Bagasse fly ash & 170.33 & Freundlich & $\begin{array}{l}\text { Pseudo } \\
\text { 2nd } \\
\text { order }\end{array}$ & - & 7.0 & $240 \mathrm{~min}$ & $1 \mathrm{~g} / \mathrm{L}$ & $\begin{array}{l}\text { (Mall et al. } \\
\text { 2005) }\end{array}$ \\
\hline 3. & Activated Slag & $\begin{array}{l}74.16 \\
\quad \text { (batch } \\
\text { capacity) } \\
78.00 \\
\text { (column } \\
\text { capacity) }\end{array}$ & $\begin{array}{l}\text { Langmuir } \\
\text { and } \\
\text { Freundlich }\end{array}$ & $\begin{array}{l}\text { Pseudo } \\
\text { 1st } \\
\text { order }\end{array}$ & Endothermic & 10.0 & $6-8 \mathrm{~h}$ & $1.0 \mathrm{~g} / \mathrm{L}$ & $\begin{array}{l}\text { (Gupta et al. } \\
\text { 1997) }\end{array}$ \\
\hline 4. & Fly ash & 40.65 & Langmuir & $\begin{array}{l}\text { Pseudo } \\
\text { 2nd } \\
\text { order }\end{array}$ & Endothermic & $>6.0$ & - & $2.0 \mathrm{~g} / \mathrm{L}$ & $\begin{array}{l}\text { (Witek- } \\
\text { Krowiak } \\
\text { et al. } \\
\text { 2012) }\end{array}$ \\
\hline 5. & $\mathrm{Ca}(\mathrm{OH})_{2}$-Treated Fly Ash & 17.383 & Langmuir & $\begin{array}{l}\text { Pseudo } \\
\text { 2nd } \\
\text { order }\end{array}$ & Exothermic & 7.0 & $120 \mathrm{~min}$ & $1.0 \mathrm{~g}$ & $\begin{array}{l}\text { (Chowdhury } \\
\text { and Saha } \\
\text { 2011a) }\end{array}$ \\
\hline 6. & Bottom ash & $6.2 \times 10^{-3}$ & Langmuir & $\begin{array}{l}\text { Pseudo } \\
\text { 2nd } \\
\text { order }\end{array}$ & Endothermic & 5.0 & $4500 \mathrm{~s}$ & $0.1 \mathrm{~g}$ & $\begin{array}{l}\text { (Gupta et al. } \\
\text { 2004) }\end{array}$ \\
\hline 7. & $\begin{array}{l}\text { Carbon based adsorbents [waste } \\
\text { material from paper industry } \\
\text { (WMPI) and pine bark (PB)] }\end{array}$ & - & - & - & - & - & - & $\begin{array}{r}0.05 \mathrm{mg} / \\
20 \mathrm{~mL}\end{array}$ & $\begin{array}{l}\text { (Méndez } \\
\text { et al. } \\
\text { 2007) }\end{array}$ \\
\hline
\end{tabular}

to both animal and vegetable derived material. The major advantages of biosorption over conventional treatment methods include, low cost; high efficiency; minimization of chemical or biological sludge; no additional nutrient requirement and possibility of regeneration of biosorbent (Volesky 1990).

Decolorization/bioadsorption of MG dye from wastewater by (dead/living) biomass of submerged aquatic plant (Rajesh Kannan et al. 2010); white-rot fungi (Kuhar and Papinutti 2013); fresh water algae (Kumar et al. 2005); brown marine algae (Kannan et al. 2009) and immobilized baker's yeast cells (Godbole and Sawant 2006) were studied by many researchers (shown in Table 8)

Wang (2009) used alligator weed (a freshwater macrophyte) as biosorbent for the removal of basic dye malachite green from aqueous solution mainly because alligator weed, originated from South America, occupies agricultural areas and blocks drainage and irrigation channels causing problems on agricultural land. In addition, it causes water pollution from plant decomposition and an increase in mosquito breeding areas. Finally, author concluded that the alligator weed could serve as low-cost adsorbents for removing MG from aqueous medium.
Parthenium (Parthenium hysterophorus L.), also known as white top or carrot weed, an annual herbaceous weed was pretreated with $\mathrm{H}_{3} \mathrm{PO}_{4}$ and $\mathrm{H}_{2} \mathrm{SO}_{4}$ by Lata et al. (2008) and employed for the adsorption of MG dye.

Biopolymers Biopolymers are high molecular weight compounds synthesized by living organisms. The performance of chitin hydrogel as an adsorbent to remove MG has been demonstrated by Tang et al. (2012). They found that the maximum adsorption capacity of hydrogel for MG was $26.88 \mathrm{mg} / \mathrm{g}$. Authors concluded from the calculated activation energy $(37.18 \mathrm{~kJ} / \mathrm{mol})$ and from the results of SEM and FTIR that the adsorption of malachite green on the hydrogel was physical process.

Bekçi et al. (2008) synthesized chitosan beads and examined its adsorption capacity towards MG. In contrast to the chitin hydrogels the authors concluded from the calculated activation energy value $(85.6 \mathrm{~kJ} / \mathrm{mol})$ that the adsorption of MG onto chitosan beads was chemical in nature.

Although chitin and chitosan are abundantly available biopolymers and are biocompatible and biodegradable material, they have less chemical versatility due to their strong crystal structure, and hence their chemical structures 
Table 7 The maximum adsorption capacities and other optimal conditions of various natural materials for adsorption of MG from aqueous solutions

\begin{tabular}{|c|c|c|c|c|c|c|c|c|c|}
\hline $\begin{array}{l}\text { Sr. } \\
\text { No. }\end{array}$ & Adsorbents & $Q_{\max }(\mathrm{mg} / \mathrm{g})$ & $\begin{array}{l}\text { Isotherm } \\
\text { study }\end{array}$ & $\begin{array}{l}\text { Kinetics } \\
\text { study }\end{array}$ & $\begin{array}{l}\text { Thermodynamic } \\
\text { study }\end{array}$ & $\mathrm{pH}$ & $\begin{array}{l}\text { Equilibrium } \\
\text { time }\end{array}$ & $\begin{array}{l}\text { Dosage of } \\
\text { adsorbent }\end{array}$ & Reference \\
\hline 1. & $\begin{array}{l}\text { Surfactant-modified } \\
\text { alumina (SMA) }\end{array}$ & 185.00 & Langmuir & - & - & - & $1 \mathrm{~h}$ & $1.0 \mathrm{~g} / \mathrm{L}$ & $\begin{array}{l}\text { (Das et al. } \\
\text { 2009a) }\end{array}$ \\
\hline 2. & $\begin{array}{l}\text { Sodium dodecyl sulfate } \\
\text { (SDS) modified } \\
\text { alumina }\end{array}$ & 185.00 & Langmuir & - & - & - & $1 \mathrm{~h}$ & $1.0 \mathrm{~g} / \mathrm{L}$ & $\begin{array}{c}\text { (Das et al. } \\
2009 b)\end{array}$ \\
\hline 3. & Bentonite & 178.60 & Langmuir & $\begin{array}{l}\text { Pseudo } \\
\text { 2nd } \\
\text { order }\end{array}$ & Endothermic & 5.0 & $60 \mathrm{~min}$ & $1 \mathrm{~g} / \mathrm{L}$ & $\begin{array}{l}\text { (Bulut et al. } \\
\text { 2008) }\end{array}$ \\
\hline 4. & $\begin{array}{l}\text { Aminopropyl } \\
\text { functionalized } \\
\text { magnesium } \\
\text { phyllosilicate (AMP) } \\
\text { clay }\end{array}$ & 130.64 & Langmuir & $\begin{array}{l}\text { Pseudo } \\
\text { 1st } \\
\text { order }\end{array}$ & Endothermic & 9.8 & $180 \mathrm{~min}$ & $\begin{array}{c}0.1 \mathrm{mg} / \\
\mathrm{mL}\end{array}$ & $\begin{array}{l}\text { (Lee et al. } \\
\text { 2011) }\end{array}$ \\
\hline 5. & $\begin{array}{l}\text { Clayey soil of Indian } \\
\text { origin }\end{array}$ & 78.57 & Langmuir & $\begin{array}{l}\text { Pseudo } \\
\text { 2nd } \\
\text { order }\end{array}$ & Exothermic & 6.0 & $90 \mathrm{~min}$ & $3 \mathrm{~g} / \mathrm{L}$ & $\begin{array}{l}\text { (Saha et al. } \\
\text { 2010b) }\end{array}$ \\
\hline 6. & Kaolin & 65.42 & Langmuir & $\begin{array}{l}\text { Pseudo } \\
\text { 2nd } \\
\text { order }\end{array}$ & Exothermic & 7.0 & $90 \min$ & $2 \mathrm{~g} / \mathrm{L}$ & $\begin{array}{l}\text { (Nandi et al. } \\
\text { 2009) }\end{array}$ \\
\hline 7. & Non-tetrahedral alumina & 61.35 & Langmuir & - & Endothermic & 5.0 & $4 \mathrm{~h}$ & $\begin{array}{r}250 \mathrm{mg} / \\
50 \mathrm{~mL}\end{array}$ & $\begin{array}{l}\text { (Kannan et al. } \\
\text { 2008) }\end{array}$ \\
\hline 8. & $\begin{array}{l}\text { Organically modified } \\
\text { clay }\end{array}$ & 56.82 & Langmuir & $\begin{array}{l}\text { Pseudo } \\
\text { 2nd } \\
\text { order }\end{array}$ & Endothermic & 6.0 & $30 \mathrm{~min}$ & & $\begin{array}{l}\text { (Arellano- } \\
\text { Cárdenas } \\
\text { et al. 2013) }\end{array}$ \\
\hline 9. & $\begin{array}{l}\text { Low-cost inorganic } \\
\text { powder (Persian } \\
\text { Kaolin) }\end{array}$ & 52.91 & Langmuir & $\begin{array}{l}\text { Pseudo } \\
\text { 2nd } \\
\text { order }\end{array}$ & Endothermic & 7.0 & $5 \mathrm{~min}$ & $0.8 \mathrm{~g} / \mathrm{L}$ & $\begin{array}{l}\text { (Tehrani- } \\
\text { Bagha et al. } \\
\text { 2011) }\end{array}$ \\
\hline 10. & Bentonite clay & 7.716 & $\begin{array}{l}\text { Langmuir, } \\
\text { Freundlich } \\
\text { and D-R }\end{array}$ & $\begin{array}{l}\text { Pseudo } \\
\text { 1st } \\
\text { order }\end{array}$ & Endothermic & 9.0 & $10 \min$ & $0.05 \mathrm{~g}$ & $\begin{array}{l}\text { (Tahir and } \\
\text { Rauf 2006) }\end{array}$ \\
\hline 11. & $\begin{array}{l}\text { Surfactant-Coated } \\
\text { Alumina (SDS- } \\
\text { Al2O3) }\end{array}$ & 2.78 & - & - & - & 4.7 & - & $500 \mathrm{mg}$ & $\begin{array}{l}\text { (Farhadi et al. } \\
\text { 2010) }\end{array}$ \\
\hline 12. & Rarasaponin-bentonite & $0.93 \mathrm{mmol} / \mathrm{g}$ & Langmuir & - & - & 8.0 & $2 \mathrm{~h}$ & - & $\begin{array}{l}\text { (Kurniawan } \\
\text { et al. 2012) }\end{array}$ \\
\hline 13. & Natural kaolinitic-clay & $0.64 \mathrm{mmol} / \mathrm{g}$ & Langmuir & - & Exothermic & 7.0 & 1 day & $1.0 \mathrm{~g} / \mathrm{L}$ & $\begin{array}{l}\text { (Issa et al. } \\
\text { 2014) }\end{array}$ \\
\hline 14. & Iron humate $(\mathrm{IH})$ & $\begin{array}{l}0.0527 \mathrm{mmol} / \\
\mathrm{g}\end{array}$ & Langmuir & - & - & - & - & - & (Janoš 2003) \\
\hline 15. & $\begin{array}{l}\text { Natural rarasaponin } \\
\text { modified kaolin }\end{array}$ & - & Sips & $\begin{array}{l}\text { Pseudo } \\
\text { 1st } \\
\text { order }\end{array}$ & Exothermic & - & - & - & $\begin{array}{l}\text { (Suwandi et al. } \\
\text { 2012) }\end{array}$ \\
\hline 16. & $\begin{array}{l}\text { Inorganic-organic } \\
\text { pillared clay granules }\end{array}$ & - & Langmuir & $\begin{array}{l}\text { Pseudo } \\
\text { 1st } \\
\text { order }\end{array}$ & - & 6.0 & $120 \mathrm{~min}$ & - & $\begin{array}{l}\text { (Cheknane } \\
\text { et al. 2010) }\end{array}$ \\
\hline 17. & Montmorillonite & - & Langmuir & - & Endothermic & - & - & $0.1 \mathrm{~g}$ & $\begin{array}{l}\text { (Tahir et al. } \\
\text { 2010) }\end{array}$ \\
\hline 18. & $\begin{array}{l}\text { Organomineral sorbent- } \\
\text { iron humate }\end{array}$ & - & - & $\begin{array}{l}\text { Pseudo } \\
\text { 2nd } \\
\text { order }\end{array}$ & - & - & - & - & $\begin{array}{l}\text { (Janoš and } \\
\text { Šmídová } \\
\text { 2005) }\end{array}$ \\
\hline 19. & Bentonite & - & $\begin{array}{l}\text { Temkin and } \\
\text { Redlich- } \\
\text { Peterson } \\
\text { isotherms }\end{array}$ & - & - & 8.2 & $60 \mathrm{~min}$ & $1.0 \mathrm{~g}$ & $\begin{array}{l}\text { (Özdemir and } \\
\text { Keskin } \\
2009)\end{array}$ \\
\hline
\end{tabular}


Table 7 continued

\begin{tabular}{|c|c|c|c|c|c|c|c|c|c|}
\hline $\begin{array}{l}\text { Sr. } \\
\text { No. }\end{array}$ & Adsorbents & $Q_{\max }(\mathrm{mg} / \mathrm{g})$ & $\begin{array}{l}\text { Isotherm } \\
\text { study }\end{array}$ & $\begin{array}{l}\text { Kinetics } \\
\text { study }\end{array}$ & $\begin{array}{l}\text { Thermodynamic } \\
\text { study }\end{array}$ & $\mathrm{pH}$ & $\begin{array}{l}\text { Equilibrium } \\
\text { time }\end{array}$ & $\begin{array}{l}\text { Dosage of } \\
\text { adsorbent }\end{array}$ & Reference \\
\hline 20. & Activated $\alpha$-alumina & - & - & - & - & 10.0 & $10 \mathrm{~min}$ & $\begin{array}{l}0.1 \mathrm{~g} / \\
\quad 250 \mathrm{~mL}\end{array}$ & $\begin{array}{l}\text { (Rogaieh and } \\
\text { Gharbani } \\
\text { 2013) }\end{array}$ \\
\hline 21. & Sand & - & Freundlich & $\begin{array}{l}\text { Pseudo } \\
\text { 2nd } \\
\text { order }\end{array}$ & - & 4.8 & - & $0.03 \mathrm{~g}$ & $\begin{array}{l}\text { (Rauf et al. } \\
\text { 2008) }\end{array}$ \\
\hline 22. & Zeolite & 46.35 & Langmuir & $\begin{array}{c}\text { Pseudo } \\
1 \text { st } \\
\text { order }\end{array}$ & - & 6.0 & - & - & $\begin{array}{l}\text { (Wang and } \\
\text { Ariyanto } \\
\text { 2007) }\end{array}$ \\
\hline 23. & Tetrahedral silica & 45.05 & Langmuir & - & Endothermic & 6.0 & $5 \mathrm{~h}$ & $\begin{array}{r}150 \mathrm{mg} / \\
50 \mathrm{~mL}\end{array}$ & $\begin{array}{l}\text { (Kannan et al. } \\
\text { 2008) }\end{array}$ \\
\hline 24. & Natural zeolite & 23.55 & - & - & - & - & - & - & $\begin{array}{c}\text { (Wu et al. } \\
2010)\end{array}$ \\
\hline 25. & Talc (silicate material) & 20.968 & Langmuir & $\begin{array}{l}\text { Pseudo } \\
\text { 2nd } \\
\text { order }\end{array}$ & - & 8.0 & - & - & $\begin{array}{l}\text { (Lee et al. } \\
\text { 2013) }\end{array}$ \\
\hline 26. & Perlite & 3.36 & Freundlich & $\begin{array}{c}\text { Pseudo } \\
1 \text { st } \\
\text { order }\end{array}$ & Exothermic & $8.0-9.0$ & $40 \min$ & $\begin{array}{l}0.1 \mathrm{~g} / \\
\quad 50 \mathrm{~mL}\end{array}$ & $\begin{array}{r}\text { (Govindasamy } \\
\text { et al. 2009) }\end{array}$ \\
\hline 27. & $\begin{array}{l}\text { Philipsite-rich-zeolite } \\
\text { tuff }\end{array}$ & $1.22 \mathrm{mmol} / \mathrm{g}$ & Langmuir & - & Exothermic & 7.0 & 1 day & $1.0 \mathrm{~g} / \mathrm{L}$ & $\begin{array}{l}\text { (Issa et al. } \\
\text { 2014) }\end{array}$ \\
\hline 28. & Natural zeolite & - & $\begin{array}{l}\text { Koble- } \\
\quad \text { Corrigan } \\
\text { model }\end{array}$ & $\begin{array}{l}\text { Pseudo } \\
\text { 2nd } \\
\text { order }\end{array}$ & Endothermic & 4.0 & $480 \mathrm{~min}$ & $\begin{array}{l}0.03 \mathrm{~g} / \\
10 \mathrm{~mL}\end{array}$ & $\begin{array}{l}\text { (Han et al. } \\
\text { 2010) }\end{array}$ \\
\hline 29. & Silica gel & - & - & - & - & - & - & - & $\begin{array}{l}\text { (Samiey and } \\
\text { Toosi 2010) }\end{array}$ \\
\hline
\end{tabular}

have been modified to overcome this undesirable characteristic. In an effort to increase the chemical versatility and adsorption efficiency, chitin and chitosan biopolymers have been modified or crosslinked by various surfactants and crosslinking agents.

Huang et al. (2012) explored the use of Chitin grafted poly (acrylic acid) (chi-g-PAA) for the removal of $\mathrm{MG}$ from wastewater and concluded that the carboxylic groups that are grafted to the surface of chitin can be hydrolyzed to a sodium salt using dilute $\mathrm{NaOH}_{(\mathrm{aq})}$ and can be readily utilized to adsorb malachite green from aqueous solution. The monolayer adsorption capacity obtained was $285.70 \mathrm{mg} / \mathrm{g}$ which was 10 times higher than the chitin hydrogels $(26.88 \mathrm{mg} / \mathrm{g})$.

Wan Ngah et al. (2010) studied the removal of MG from aqueous solutions through adsorption on to chitosan coated bentonite beads. The characterization of adsorbents was performed by Fourier Transform Infrared (FTIR) spectroscopy, scanning electron microscopy (SEM) coupled with energy dispersive spectroscopy (EDS) and X-ray diffraction (XRD) analysis. Authors mentioned that the monolayer adsorption capacity obtained was $435.00 \mathrm{mg} / \mathrm{g}$ which was five times greater than the chitosan beads (93.55 mg/g).
Peat Peat is a porous and complex natural material, widely available and studied as an alternative adsorbent for different pollutants as well as dyes (Raval et al. 2016c).

Coco-peat was used by Vijayaraghavan et al. (2015) as an adsorbent to study the adsorption of malachite green and crystal violet from wastewater. It was found that a contact time of $60 \mathrm{~min}$ was sufficient for the adsorption to reach equilibrium. The optimum $\mathrm{pH}$ was found to be 7.0. The data for the adsorption of MG was described well by both the Sips adsorption isotherm model. The adsorption process was found to be endothermic.

\section{Nanomaterials}

Typical nanomaterials include nanotubes, nanoparticles, nanofibers, nanorods, fullerenes, and nanowires (Schmidt et al. 2002; Gupta and Saleh 2013; Gupta and Nayak 2012; Saravanan et al. 2013, 2014).

Carbon nanotubes were discovered accidentally by Iijima (1991), while studying the surfaces of graphite electrodes used in an electric arc discharge. CNTs are divided into two types based on the arrangement of their graphene cylinders: (1) single-walled CNTs (SWCNTs) 
Table 8 The maximum adsorption capacities and other optimal conditions of various biosorbents for adsorption of MG from water and wastewater

\begin{tabular}{|c|c|c|c|c|c|c|c|c|c|}
\hline $\begin{array}{l}\text { Sr. } \\
\text { No. }\end{array}$ & Adsorbents & $\begin{array}{l}Q_{\max } \\
(\mathrm{mg} / \mathrm{g})\end{array}$ & $\begin{array}{l}\text { Isotherm } \\
\text { study }\end{array}$ & $\begin{array}{l}\text { Kinetics } \\
\text { study }\end{array}$ & $\begin{array}{l}\text { Thermodynamic } \\
\text { study }\end{array}$ & $\mathrm{pH}$ & $\begin{array}{l}\text { Equilibrium } \\
\text { time }\end{array}$ & $\begin{array}{l}\text { Dosage of } \\
\text { adsorbent }\end{array}$ & References \\
\hline 1. & $\begin{array}{l}\text { Freshwater macrophyte } \\
\text { alligator weed }\end{array}$ & 185.54 & Langmuir & $\begin{array}{l}\text { Ritchie } \\
\text { second- } \\
\text { order }\end{array}$ & - & 6.0 & - & $7.0 \mathrm{~g} / \mathrm{L}$ & (Wang 2009) \\
\hline 2. & $\begin{array}{l}\text { Firmiana simplex wood } \\
\text { fiber }\end{array}$ & $>142.40$ & Freundlich & $\begin{array}{l}\text { Pseudo 2nd } \\
\text { order }\end{array}$ & Endothermic & 11.0 & $180 \mathrm{~min}$ & $0.25 \mathrm{~g}$ & $\begin{array}{l}\text { (Pan and Zhang } \\
\text { 2009) }\end{array}$ \\
\hline 3. & $\begin{array}{l}\text { Pleurotus ostreatus (A } \\
\text { macro-fungus) }\end{array}$ & 125.00 & Freundlich & $\begin{array}{l}\text { Pseudo 2nd } \\
\text { order }\end{array}$ & - & 8.0 & $240 \mathrm{~min}$ & $4.0 \mathrm{~g} / \mathrm{L}$ & $\begin{array}{l}\text { (Chen et al. } \\
\text { 2014) }\end{array}$ \\
\hline 4. & $\begin{array}{l}\text { Pithophora sp., a fresh } \\
\text { water algae }\end{array}$ & 117.65 & $\begin{array}{l}\text { Redlich- } \\
\text { Peterson }\end{array}$ & $\begin{array}{l}\text { Pseudo 2nd } \\
\text { order }\end{array}$ & - & 5.0 & $8 \mathrm{~h}$ & $0.015 \mathrm{~g}$ & $\begin{array}{l}\text { (Kumar et al. } \\
\text { 2005) }\end{array}$ \\
\hline 5. & $\begin{array}{l}\text { Hydrilla verticillata } \\
\text { biomass }\end{array}$ & 91.97 & Freundlich & $\begin{array}{l}\text { Pseudo 2nd } \\
\text { order }\end{array}$ & - & 8.0 & $200 \mathrm{~min}$ & $0.26 \mathrm{~g}$ & $\begin{array}{l}\text { (Rajeshkannan } \\
\text { et al. 2010) }\end{array}$ \\
\hline 6. & $\begin{array}{c}\text { Ashoka (Saraca asoca) } \\
\text { leaf powder (ALP) }\end{array}$ & 83.30 & Freundlich & $\begin{array}{l}\text { Pseudo 2nd } \\
\text { order }\end{array}$ & Exothermic & 6.0 & $25 \mathrm{~min}$ & $2.0 \mathrm{~g} / \mathrm{L}$ & $\begin{array}{l}\text { (Gupta et al. } \\
\text { 2012) }\end{array}$ \\
\hline 7. & $\begin{array}{l}\text { Hydrilla Verticillata } \\
\text { Biomass }\end{array}$ & 69.88 & Langmuir & $\begin{array}{l}\text { Pseudo 1st } \\
\text { order }\end{array}$ & - & 8.0 & $150 \mathrm{~min}$ & $0.55 \mathrm{~g}$ & $\begin{array}{l}\text { (Rajesh Kannan } \\
\text { et al. 2010) }\end{array}$ \\
\hline 8. & $\begin{array}{l}\text { Turbinaria conoides } \\
\text { brown marine algae }\end{array}$ & 66.60 & Langmuir & $\begin{array}{l}\text { Pseudo 2nd } \\
\text { order }\end{array}$ & - & 8.0 & $150 \mathrm{~min}$ & $0.55 \mathrm{~g}$ & $\begin{array}{l}\text { (Kannan et al. } \\
\text { 2009) }\end{array}$ \\
\hline 9. & $\begin{array}{l}\text { Native anaerobic granular } \\
\text { sludge }\end{array}$ & 61.73 & Langmuir & $\begin{array}{l}\text { Pseudo 2nd } \\
\quad \text { order }\end{array}$ & Exothermic & 5.0 & $2 \mathrm{~h}$ & $2.4 \mathrm{~g} / \mathrm{L}$ & $\begin{array}{l}\text { (Cheng et al. } \\
\text { 2008) }\end{array}$ \\
\hline 10. & $\begin{array}{l}\text { Heat-treated anaerobic } \\
\text { granular sludge }\end{array}$ & 59.17 & Langmuir & $\begin{array}{l}\text { Pseudo 2nd } \\
\quad \text { order }\end{array}$ & Exothermic & 5.0 & $2 \mathrm{~h}$ & $2.4 \mathrm{~g} / \mathrm{L}$ & $\begin{array}{l}\text { (Cheng et al. } \\
\text { 2008) }\end{array}$ \\
\hline 11. & $\begin{array}{l}\text { Eriobotrya japonica } \\
\text { (Thunb.) Lindley } \\
\text { (Loquat) seed biomass }\end{array}$ & 57.47 & Langmuir & $\begin{array}{l}\text { Pseudo 2nd } \\
\text { order }\end{array}$ & Endothermic & 5.0 & $60 \mathrm{~min}$ & $10.0 \mathrm{~g} / \mathrm{L}$ & $\begin{array}{l}\text { (Aksakal et al. } \\
\text { 2009) }\end{array}$ \\
\hline 12. & $\begin{array}{l}\text { Daucus carota plant } \\
\text { (carrot leaves powder: } \\
\text { CLP) }\end{array}$ & 52.60 & Freundlich & $\begin{array}{l}\text { Pseudo 2nd } \\
\quad \text { order }\end{array}$ & Exothermic & 7.0 & $30 \mathrm{~min}$ & $2.0 \mathrm{~g} / \mathrm{L}$ & $\begin{array}{l}\text { (Kushwaha et al. } \\
\text { 2014) }\end{array}$ \\
\hline 13. & $\begin{array}{l}\text { Mango (Mangifera indica } \\
\text { L.) seed husks [MSH] }\end{array}$ & 47.90 & Freundlich & $\begin{array}{l}\text { Pseudo 2nd } \\
\text { order }\end{array}$ & Exothermic & 5.0 & $2.0 \mathrm{~h}$ & $5.0 \mathrm{~g} / \mathrm{L}$ & $\begin{array}{l}\text { (Franca et al. } \\
\text { 2010b) }\end{array}$ \\
\hline 14. & $\begin{array}{l}\text { Daucus carota plant } \\
\text { (carrot stem powder: } \\
\text { CSP) }\end{array}$ & 43.40 & Freundlich & $\begin{array}{l}\text { Pseudo 2nd } \\
\text { order }\end{array}$ & Exothermic & 7.0 & $30 \mathrm{~min}$ & $2.0 \mathrm{~g} / \mathrm{L}$ & $\begin{array}{l}\text { (Kushwaha et al. } \\
\text { 2014) }\end{array}$ \\
\hline 15. & $\begin{array}{l}\text { Dead needles of Aleppo } \\
\text { pine (Pinus halepensis) }\end{array}$ & 33.56 & Freundlich & $\begin{array}{l}\text { Intraparticle } \\
\text { diffusion }\end{array}$ & Endothermic & - & $330 \mathrm{~min}$ & $\begin{array}{l}0.5 \mathrm{~g} / \\
\quad 300 \mathrm{~mL}\end{array}$ & $\begin{array}{l}\text { (Hamdaoui et al. } \\
\text { 2008a) }\end{array}$ \\
\hline 16. & $\begin{array}{l}\text { Almond Shell (Prunus } \\
\text { dulcis) }\end{array}$ & 29.0 & Langmuir & $\begin{array}{l}\text { Pseudo 2nd } \\
\quad \text { order }\end{array}$ & Endothermic & - & $180 \mathrm{~min}$ & $20 \mathrm{~g} / \mathrm{L}$ & $\begin{array}{l}\text { (Ozdes et al. } \\
\text { 2010) }\end{array}$ \\
\hline 17. & $\begin{array}{l}\text { Phosphoric acid treated } \\
\text { Parthenium } \\
\text { hysterophorus Linn. } \\
\text { Biomass (PWC) }\end{array}$ & 25.00 & Langmuir & $\begin{array}{l}\text { Pseudo 2nd } \\
\text { order }\end{array}$ & - & 7.0 & $90 \min$ & $\begin{array}{l}0.2 \mathrm{~g} / \\
50 \mathrm{~mL}\end{array}$ & (Lata et al. 2008) \\
\hline 18. & $\begin{array}{l}\text { Microwave assisted zinc } \\
\text { chloride activated } \\
\text { epicarp of Ricinus } \\
\text { communis (ZRC) }\end{array}$ & 24.39 & Langmuir & $\begin{array}{l}\text { Pseudo 2nd } \\
\text { order }\end{array}$ & - & 5.0 & & $0.2 \mathrm{~g} / \mathrm{mL}$ & $\begin{array}{l}\text { (Makeswari and } \\
\text { Santhi 2013) }\end{array}$ \\
\hline 19. & $\begin{array}{l}\text { Immobilized } \\
\text { Saccharomyces } \\
\text { cerevisiae }\end{array}$ & 17.0 & Freundlich & - & - & - & - & - & $\begin{array}{l}\text { (Godbole and } \\
\text { Sawant 2006) }\end{array}$ \\
\hline 20. & $\begin{array}{l}\text { Sulfuric acid treated } \\
\text { Parthenium } \\
\text { hysterophorus Linn. } \\
\text { Biomass }\end{array}$ & 16.81 & Langmuir & $\begin{array}{l}\text { Pseudo 2nd } \\
\text { order }\end{array}$ & - & 7.0 & $90 \mathrm{~min}$ & $\begin{array}{l}0.2 \mathrm{~g} / \\
50 \mathrm{~mL}\end{array}$ & (Lata et al. 2008) \\
\hline 21. & $\begin{array}{c}\text { Microwave activated } \\
\text { epicarp of Ricinus } \\
\text { communis (MRC) }\end{array}$ & 12.65 & Langmuir & $\begin{array}{l}\text { Pseudo 2nd } \\
\text { order }\end{array}$ & - & 5.0 & - & $0.2 \mathrm{~g} / \mathrm{mL}$ & $\begin{array}{l}\text { (Makeswari and } \\
\text { Santhi 2013) }\end{array}$ \\
\hline
\end{tabular}


Table 8 continued

\begin{tabular}{|c|c|c|c|c|c|c|c|c|c|}
\hline $\begin{array}{l}\text { Sr. } \\
\text { No. }\end{array}$ & Adsorbents & $\begin{array}{l}Q_{\max } \\
(\mathrm{mg} / \mathrm{g})\end{array}$ & $\begin{array}{l}\text { Isotherm } \\
\text { study }\end{array}$ & $\begin{array}{l}\text { Kinetics } \\
\text { study }\end{array}$ & $\begin{array}{l}\text { Thermodynamic } \\
\text { study }\end{array}$ & $\mathrm{pH}$ & $\begin{array}{l}\text { Equilibrium } \\
\text { time }\end{array}$ & $\begin{array}{l}\text { Dosage of } \\
\text { adsorbent }\end{array}$ & References \\
\hline 22. & $\begin{array}{l}\text { Hydrilla verticillata } \\
\text { biomass }\end{array}$ & - & - & - & - & 8.4 & $194.50 \mathrm{~min}$ & $11.14 \mathrm{~g} / \mathrm{L}$ & $\begin{array}{c}\text { (Rajeshkannan } \\
\text { et al. 2009) }\end{array}$ \\
\hline 23. & $\begin{array}{l}\text { Pithophora sp., a fresh } \\
\text { water algae }\end{array}$ & - & - & $\begin{array}{l}\text { Pseudo 1st } \\
\text { order }\end{array}$ & - & 6.0 & - & - & $\begin{array}{l}\text { (Vasanth Kumar } \\
\text { et al. 2006) }\end{array}$ \\
\hline 24. & $\begin{array}{l}\text { Yeast } S \text {. cerevisiae MTCC } \\
\quad 174\end{array}$ & - & - & $\begin{array}{l}\text { Pseudo 2nd } \\
\text { order }\end{array}$ & Endothermic & 6.88 & $60 \mathrm{~min}$ & $0.49 \mathrm{~g}$ & $\begin{array}{l}\text { (Singh et al. } \\
\text { 2012) }\end{array}$ \\
\hline 25. & Chlorella-based biomass & - & - & $\begin{array}{l}\text { Pseudo 2nd } \\
\text { order }\end{array}$ & - & 7.0 & & $\begin{array}{l}2.0 \mathrm{~g} / \\
2.0 \mathrm{dm}^{3}\end{array}$ & $\begin{array}{l}\text { (Tsai and Chen } \\
\text { 2010) }\end{array}$ \\
\hline 26. & $\begin{array}{l}\text { White-rot fungus } \\
\text { Ganoderma lucidum }\end{array}$ & - & - & - & - & - & - & - & $\begin{array}{l}\text { (Kuhar and } \\
\text { Papinutti } \\
\text { 2013) }\end{array}$ \\
\hline 27. & $\begin{array}{l}\text { Carboxymethyl cellulose- } \\
\text { acrylic acid }\end{array}$ & 555.56 & Temkin & $\begin{array}{l}\text { Pseudo 2nd } \\
\text { order }\end{array}$ & Endothermic & - & $\begin{array}{l}120 \mathrm{~min} \\
(1 \mathrm{~h})\end{array}$ & - & $\begin{array}{l}\text { (Zhang et al. } \\
\text { 2014a) }\end{array}$ \\
\hline 28. & $\begin{array}{l}\text { Chitosan coated bentonite } \\
\text { (CCB) beads }\end{array}$ & 435.0 & Freundlich & $\begin{array}{l}\text { Pseudo 2nd } \\
\text { order }\end{array}$ & - & 6.0 & $30 \mathrm{~min}$ & $\begin{array}{l}0.01 \mathrm{~g} / \\
50 \mathrm{~mL}\end{array}$ & $\begin{array}{l}\text { (Wan Ngah et al. } \\
\text { 2010) }\end{array}$ \\
\hline 29. & $\begin{array}{l}\text { Cellulose modified with } \\
\text { maleic anhydride } \\
\text { (CMA) }\end{array}$ & 370.00 & Langmuir & $\begin{array}{l}\text { Pseudo 2nd } \\
\text { order }\end{array}$ & Exothermic & - & - & - & $\begin{array}{l}\text { (Zhou et al. } \\
\text { 2015) }\end{array}$ \\
\hline 30. & $\begin{array}{l}\text { Amylopectin and poly } \\
\text { (acrylic acid) (AP-g- } \\
\text { PAA) }\end{array}$ & 352.11 & Langmuir & $\begin{array}{l}\text { Pseudo 2nd } \\
\text { order }\end{array}$ & Endothermic & 7.0 & $30 \mathrm{~min}$ & $50 \mathrm{mg}$ & $\begin{array}{l}\text { (Sarkar et al. } \\
\text { 2014) }\end{array}$ \\
\hline 31. & $\begin{array}{l}\text { Chitin grafted poly } \\
\text { (acrylic acid) }\end{array}$ & 285.70 & Langmuir & - & - & - & - & - & $\begin{array}{l}\text { (Huang et al. } \\
\text { 2012) }\end{array}$ \\
\hline 32. & Chitosan beads & 93.55 & Langmuir & $\begin{array}{l}\text { Pseudo 2nd } \\
\text { order }\end{array}$ & Endothermic & 8.0 & $\begin{array}{l}300 \mathrm{~min} \\
(5 \mathrm{~h})\end{array}$ & $10 \mathrm{~g} / \mathrm{L}$ & $\begin{array}{l}\text { (Bekçi et al. } \\
\text { 2008) }\end{array}$ \\
\hline 33. & $\begin{array}{l}\text { Cyclodextrin-based } \\
\text { adsorbent }\end{array}$ & 91.90 & Langmuir & $\begin{array}{l}\text { Pseudo 2nd } \\
\text { order }\end{array}$ & - & 8.0 & $120 \mathrm{~min}$ & $0.15 \mathrm{~g}$ & $\begin{array}{l}\text { (Crini et al. } \\
\text { 2007) }\end{array}$ \\
\hline 34. & Lignin sulfonate polymer & 70.077 & Langmuir & $\begin{array}{l}\text { Pseudo 2nd } \\
\quad \text { order }\end{array}$ & Endothermic & 7.0 & $300 \mathrm{~min}$ & $0.05 \mathrm{~g}$ & $\begin{array}{l}\text { (Tang et al. } \\
\text { 2014) }\end{array}$ \\
\hline 35. & Bio-polymer (Cellulose) & 2.422 & $\begin{array}{l}\text { Langmuir } \\
\text { and } \\
\text { Freundlich }\end{array}$ & $\begin{array}{l}\text { Pseudo 2nd } \\
\text { order }\end{array}$ & Exothermic & 7.2 & 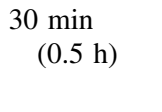 & $5 \mathrm{~g} / \mathrm{L}$ & $\begin{array}{l}\text { (Pradeep Sekhar } \\
\text { et al. 2009) }\end{array}$ \\
\hline 36. & Chitin hydrogels & 26.883 & Langmuir & $\begin{array}{l}\text { Pseudo 2nd } \\
\text { order }\end{array}$ & Endothermic & 7.0 & $25 \mathrm{~h}$ & - & $\begin{array}{l}\text { (Tang et al. } \\
\text { 2012) }\end{array}$ \\
\hline 37. & Coco-peat & 276.80 & Sips & $\begin{array}{l}\text { Pseudo 1st } \\
\text { order }\end{array}$ & Endothermic & 7.0 & $60 \mathrm{~min}$ & $\begin{array}{l}0.2 \mathrm{~g} / \\
100 \mathrm{~mL}\end{array}$ & $\begin{array}{c}\text { (Vijayaraghavan } \\
\text { et al. 2015) }\end{array}$ \\
\hline 38. & $\begin{array}{l}\text { Peat of Brunei } \\
\text { Darussalam (Keramut } \\
\text { peat) }\end{array}$ & $\begin{array}{l}0.31 \mathrm{mmol} / \\
\mathrm{g}\end{array}$ & $\begin{array}{l}\text { Redlich- } \\
\text { Peterson } \\
\text { (R-P) } \\
\text { model }\end{array}$ & $\begin{array}{l}\text { Pseudo 2nd } \\
\text { order }\end{array}$ & Endothermic & 6.0 & $4 \mathrm{~h}$ & $0.050 \mathrm{~g}$ & $\begin{array}{l}\text { (Chieng et al. } \\
\text { 2014) }\end{array}$ \\
\hline
\end{tabular}

and (2) multi-walled CNTs (MWCNTs) (Khani et al. 2010; Saleh and Gupta 2011).

MWNTs are collections of several concentric graphene cylinders and are larger structures compared to SWNTs which are individual cylinders of 1-2 nm diameter. The former can be considered as a mesoscale graphite system, whereas the latter is truly a single large molecule (Odom et al. 1998). The mechanisms by which the dye molecules are sorbed onto CNTs are very complicated and appear attributable to electrostatic attraction, sorption, precipitation and chemical interaction between the reactive groups of dye molecules and the surface functional groups of CNTs.

Recently, Rajabi et al. (2016) functionalized MWCNTs with the carboxylate group and used as an adsorbent for the rapid and fast removal of $\mathrm{MG}$ dye from the aqueous solutions. The optimized contact time and $\mathrm{pH}$ were $10 \mathrm{~min}$ and 9, respectively. The effect of temperature revealed that the adsorption capacity of $\mathrm{MG}$ dye increased with increasing contact time, temperature and $\mathrm{pH}$. The 
Table 9 The maximum adsorption capacities and other optimal conditions of various nanomaterials, composites and nanocomposite adsorbents for the removal of MG from water and wastewater

\begin{tabular}{|c|c|c|c|c|c|c|c|c|c|}
\hline $\begin{array}{l}\text { Sr. } \\
\text { No. }\end{array}$ & Adsorbents & $\begin{array}{l}Q_{\max } \\
(\mathrm{mg} / \mathrm{g})\end{array}$ & $\begin{array}{l}\text { Isotherm } \\
\text { study }\end{array}$ & $\begin{array}{l}\text { Kinetics } \\
\text { study }\end{array}$ & $\begin{array}{l}\text { Thermodynamic } \\
\text { study }\end{array}$ & $\mathrm{pH}$ & $\begin{array}{l}\text { Equilibrium } \\
\text { time }\end{array}$ & $\begin{array}{l}\text { Dosage of } \\
\text { adsorbent }\end{array}$ & References \\
\hline 1. & $\begin{array}{l}\text { Copper nanowires loaded on } \\
\text { activated carbon }\end{array}$ & 434.80 & Langmuir & $\begin{array}{l}\text { Pseudo } \\
\text { 2nd } \\
\text { order }\end{array}$ & - & 5.0 & $20 \mathrm{~min}$ & $0.1 \mathrm{~g}$ & $\begin{array}{l}\text { (Ghaedi et al. } \\
\text { 2015) }\end{array}$ \\
\hline 2. & $\begin{array}{l}\text { Zinc oxide nanoparticle } \\
\text { loaded on activated carbon } \\
\text { (ZnO-NP-AC) }\end{array}$ & 322.58 & Langmuir & $\begin{array}{l}\text { Pseudo } \\
\text { 2nd } \\
\text { order }\end{array}$ & - & 7.0 & $15 \mathrm{~min}$ & $0.005 \mathrm{~g}$ & $\begin{array}{l}\text { (Ghaedi et al. } \\
\text { 2014) }\end{array}$ \\
\hline 3. & $\begin{array}{l}\text { Metal oxide nanoparticles } \\
\quad(\mathrm{ZnO})\end{array}$ & 310.50 & $\begin{array}{l}\text { Langmuir } \\
\text { and } \\
\text { Freundlich }\end{array}$ & $\begin{array}{l}\text { Pseudo } \\
\text { 2nd } \\
\text { order }\end{array}$ & Endothermic & 8.0 & $120 \mathrm{~min}$ & & $\begin{array}{l}\text { (Kumar et al. } \\
\text { 2013) }\end{array}$ \\
\hline 4. & $\begin{array}{l}\text { Maghemite nanoparticles }(\gamma- \\
\left.\mathrm{Fe}_{2} \mathrm{O}_{3}\right)\end{array}$ & 227.30 & Langmuir & - & - & 3.0 & - & $0.10 \mathrm{~g}$ & $\begin{array}{l}\text { (Afkhami et al. } \\
\text { 2010) }\end{array}$ \\
\hline 5. & $\begin{array}{l}\text { Metal oxide nanoparticles } \\
\quad\left(\mathrm{SnO}_{2}\right)\end{array}$ & 216.90 & $\begin{array}{l}\text { Langmuir } \\
\text { and } \\
\text { Freundlich }\end{array}$ & $\begin{array}{l}\text { Pseudo } \\
\text { 2nd } \\
\text { order }\end{array}$ & Endothermic & 8.0 & $120 \mathrm{~min}$ & & $\begin{array}{l}\text { (Kumar et al. } \\
\text { 2013) }\end{array}$ \\
\hline 6. & $\begin{array}{l}\text { Gold nanoparticles loaded on } \\
\text { activated carbon (Au-NP- } \\
\text { AC) }\end{array}$ & 164.57 & Langmuir & $\begin{array}{l}\text { Pseudo } \\
\text { 2nd } \\
\text { order }\end{array}$ & Endothermic & 7.0 & $4.4 \mathrm{~min}$ & $0.015 \mathrm{~g}$ & $\begin{array}{l}\text { (Roosta et al. } \\
\text { 2014) }\end{array}$ \\
\hline 7. & $\begin{array}{l}N \text {-Benzyl-O- } \\
\text { carboxymethylchitosan } \\
\text { magnetic nanoparticles } \\
\text { (OC-BzM) }\end{array}$ & 144.79 & $\begin{array}{l}\text { Langmuir } \\
\text { and } \\
\text { Freundlich }\end{array}$ & $\begin{array}{l}\text { Pseudo } \\
\text { 2nd } \\
\text { order }\end{array}$ & Endothermic & 7.0 & $60 \mathrm{~min}$ & $25 \mathrm{mg}$ & $\begin{array}{l}\text { (Debrassi et al. } \\
\text { 2012) }\end{array}$ \\
\hline 8. & $\begin{array}{l}\text { Functionalized multi wall } \\
\text { carbon nanotubes }(f- \\
\text { MWCNTs) }\end{array}$ & 142.85 & Langmuir & $\begin{array}{l}\text { Pseudo } \\
\text { 2nd } \\
\text { order }\end{array}$ & - & 7.0 & $80 \mathrm{~min}$ & $\begin{array}{l}0.08 \mathrm{~g} / \\
100 \mathrm{~mL}\end{array}$ & $\begin{array}{l}\text { (Shirmardi et al. } \\
\text { 2013) }\end{array}$ \\
\hline 9. & $\begin{array}{l}\mathrm{NiO} \text { nano flake-based } \\
\text { flowerlike architectures }\end{array}$ & 142.08 & Langmuir & $\begin{array}{l}\text { Pseudo } \\
\text { 2nd } \\
\text { order }\end{array}$ & - & & & $\begin{array}{l}333.3 \mathrm{mg} / \\
\mathrm{L}\end{array}$ & $\begin{array}{l}\text { (Wei et al. } \\
\text { 2014) }\end{array}$ \\
\hline 10. & $\begin{array}{l}\text { Silver nano particles (AgOH- } \\
\mathrm{NP}) \text { loaded on activated } \\
\text { carbon prepared from Rosa } \\
\text { canina tree (AgOH-NP- } \\
\text { AC-RC) }\end{array}$ & 105.00 & Freundlich & $\begin{array}{l}\text { Pseudo } \\
\text { 2nd } \\
\text { order }\end{array}$ & - & 8.0 & $20 \min$ & $\begin{array}{l}0.09 \mathrm{~g} / \\
50 \mathrm{~mL}\end{array}$ & $\begin{array}{l}\text { (Tavakol et al. } \\
\text { 2015) }\end{array}$ \\
\hline 11. & $\begin{array}{l}\text { Superparamagnetic sodium } \\
\text { alginate-coated } \mathrm{Fe}_{3} \mathrm{O}_{4} \\
\text { nanoparticles }\left(\mathrm{Alg}-\mathrm{Fe}_{3} \mathrm{O}_{4}\right)\end{array}$ & 47.84 & Langmuir & $\begin{array}{l}\text { Pseudo } \\
\text { 2nd } \\
\text { order }\end{array}$ & Endothermic & 9.0 & $20 \mathrm{~min}$ & $\begin{array}{l}0.1 \mathrm{~g} / \\
50 \mathrm{~mL}\end{array}$ & $\begin{array}{r}\text { (Mohammadi } \\
\text { et al. 2014) }\end{array}$ \\
\hline 12. & $\begin{array}{l}\text { Cadmium hydroxide } \\
\text { nanowires loaded on } \\
\text { activated carbon } \\
\left(\mathrm{Cd}(\mathrm{OH})_{2}-\mathrm{NW}-\mathrm{AC}\right)\end{array}$ & 19.0 & Langmuir & $\begin{array}{l}\text { Pseudo } \\
\text { 2nd } \\
\text { order }\end{array}$ & Endothermic & - & - & - & $\begin{array}{l}\text { (Ghaedi and } \\
\text { Mosallanejad } \\
\text { 2014) }\end{array}$ \\
\hline 13. & $\begin{array}{l}\text { Melamine supported } \\
\text { magnetic iron oxide } \\
\text { nanoparticles } \\
\left(\mathrm{Fe}_{3} \mathrm{O}_{4} @ \mathrm{Mel}\right)\end{array}$ & 9.06 & Freundlich & $\begin{array}{l}\text { Pseudo } \\
\text { 2nd } \\
\text { order }\end{array}$ & - & 6.5 & $240 \mathrm{~min}$ & $0.3 \mathrm{~g}$ & $\begin{array}{l}\text { (Mirzajani and } \\
\text { Ahmadi } \\
\text { 2015) }\end{array}$ \\
\hline 14. & $\begin{array}{l}\text { Citrate stabilized magnetite } \\
\text { nanoparticles }\end{array}$ & 0.704 & Freundlich & $\begin{array}{l}\text { Pseudo } \\
\text { 2nd } \\
\text { order }\end{array}$ & - & 4.0 & - & $10 \mathrm{mg}$ & $\begin{array}{l}\text { (Mishra et al. } \\
\text { 2014) }\end{array}$ \\
\hline 15. & $\begin{array}{l}\mathrm{FeCl}_{2} / \mathrm{FeCl}_{3} / \text { perlite magnetic } \\
\text { nanoparticles }\left(\mathrm{FeCl}_{2} /\right. \\
\left.\mathrm{FeCl}_{3} / \mathrm{PMNs}\right)\end{array}$ & 0.01 & BET & $\begin{array}{l}\text { Pseudo } \\
\text { 2nd } \\
\text { order }\end{array}$ & - & 6.0 & $120 \mathrm{~min}$ & $0.05 \mathrm{~g}$ & $\begin{array}{l}\text { (Heydartaemeh } \\
\text { et al. 2014) }\end{array}$ \\
\hline 16. & $\mathrm{MnO}_{2}$ nanosheets & - & - & $\begin{array}{l}\text { Pseudo } \\
\text { 1st } \\
\text { order }\end{array}$ & - & 5.6 & $120 \mathrm{~min}$ & $0.5 \mathrm{~g} / \mathrm{L}$ & $\begin{array}{l}\text { (Saha and Pal } \\
\text { 2014) }\end{array}$ \\
\hline 17. & $\begin{array}{l}\text { Green tea extract based iron } \\
\text { nanoparticles (GT-Fe NPs) }\end{array}$ & - & - & $\begin{array}{l}\text { Pseudo } \\
1 \text { st } \\
\text { order }\end{array}$ & - & 9.0 & - & $1.12 \mathrm{~g} / \mathrm{L}$ & $\begin{array}{l}\text { (Weng et al. } \\
\text { 2013) }\end{array}$ \\
\hline
\end{tabular}


Table 9 continued

\begin{tabular}{|c|c|c|c|c|c|c|c|c|c|}
\hline $\begin{array}{l}\text { Sr. } \\
\text { No. }\end{array}$ & Adsorbents & $\begin{array}{l}Q_{\max } \\
(\mathrm{mg} / \mathrm{g})\end{array}$ & $\begin{array}{l}\text { Isotherm } \\
\text { study }\end{array}$ & $\begin{array}{l}\text { Kinetics } \\
\text { study }\end{array}$ & $\begin{array}{l}\text { Thermodynamic } \\
\text { study }\end{array}$ & $\mathrm{pH}$ & $\begin{array}{l}\text { Equilibrium } \\
\text { time }\end{array}$ & $\begin{array}{l}\text { Dosage of } \\
\text { adsorbent }\end{array}$ & References \\
\hline 18. & $\mathrm{TiO}_{2}$ nanoparticles & - & - & $\begin{array}{l}\text { Pseudo } \\
\text { 2nd } \\
\text { order }\end{array}$ & - & 6.9 & - & $3.0 \mathrm{~g} / \mathrm{L}$ & $\begin{array}{l}\text { (Suresh and } \\
\text { Annadurai } \\
\text { 2013) }\end{array}$ \\
\hline 19. & Iron nanoparticles (Fe NPs) & - & - & - & - & - & - & - & $\begin{array}{l}\text { (Huang et al. } \\
\text { 2014) }\end{array}$ \\
\hline 20. & $\begin{array}{l}\text { Reduced graphene oxide } \\
\quad(\mathrm{RGO})- \\
\mathrm{Fe}_{3} \mathrm{O}_{4} \text { nanoparticles (NPs) }\end{array}$ & - & - & - & - & - & - & - & $\begin{array}{l}\text { (Geng et al. } \\
\text { 2012) }\end{array}$ \\
\hline 21. & Halloysite nanotubes (HNTs) & 99.60 & Langmuir & $\begin{array}{l}\text { Pseudo } \\
\text { 2nd } \\
\text { order }\end{array}$ & Endothermic & 9.5 & $30 \mathrm{~min}$ & $0.2 \mathrm{~g}$ & $\begin{array}{l}\text { (Kiani et al. } \\
\text { 2011) }\end{array}$ \\
\hline 22. & $\mathrm{ZnS}$ & $98.3 \%$ & - & - & - & 8.0 & $120 \mathrm{~min}$ & $0.08 \mathrm{~g} / \mathrm{L}$ & $\begin{array}{l}\text { (Rajabi et al. } \\
\text { 2013) }\end{array}$ \\
\hline 23. & $\mathrm{ZnS}: \mathrm{Fe}$ & $99.0 \%$ & - & - & - & 8.0 & $120 \mathrm{~min}$ & $0.08 \mathrm{~g} / \mathrm{L}$ & $\begin{array}{l}\text { (Rajabi et al. } \\
\text { 2013) }\end{array}$ \\
\hline 24. & Fe3O4@SiO2-NH2 & - & Freundlich & $\begin{array}{l}\text { Pseudo } \\
\text { 2nd } \\
\text { order }\end{array}$ & - & 7.0 & - & $100 \mathrm{mg} / \mathrm{L}$ & $\begin{array}{l}\text { (Sun et al. } \\
\text { 2015b) }\end{array}$ \\
\hline 25. & Lac-PAM-CTS & - & - & - & - & - & - & - & $\begin{array}{l}\text { (Sun et al. } \\
\text { 2015a) }\end{array}$ \\
\hline 26. & $\begin{array}{l}\text { Magnetic } \beta \text {-cyclodextrin- } \\
\text { graphene oxide } \\
\text { nanocomposites }\left(\mathrm{Fe}_{3} \mathrm{O}_{4} / \beta \text { - }\right. \\
\mathrm{CD} / \mathrm{GO})\end{array}$ & 990.10 & Langmuir & $\begin{array}{l}\text { Pseudo } \\
\text { 2nd } \\
\text { order }\end{array}$ & Endothermic & 7.0 & $120 \mathrm{~min}$ & - & $\begin{array}{l}\text { (Wang et al. } \\
\text { 2015) }\end{array}$ \\
\hline 27. & K-Carrageenan-g-AA & 833.33 & Langmuir & $\begin{array}{l}\text { Pseudo } \\
\text { 2nd } \\
\text { order }\end{array}$ & Exothermic & 7.0 & $180 \mathrm{~min}$ & $0.05 \mathrm{~g}$ & $\begin{array}{l}\text { (Pourjavadi } \\
\quad \text { et al. 2014) }\end{array}$ \\
\hline 28. & $\begin{array}{l}\text { Kappa-carrageenan-g- } \\
\text { polyacrylic acid/TiO } \\
\text { hydrogel nanocomposite } \\
(\mathrm{K}-\text {-Carrageenan-g-AA/ } \\
\left.\mathrm{TiO}_{2}-\mathrm{NH}_{2}\right)\end{array}$ & 666.66 & Langmuir & $\begin{array}{l}\text { Pseudo } \\
\text { 2nd } \\
\text { order }\end{array}$ & Exothermic & 7.0 & $180 \min$ & $0.05 \mathrm{~g}$ & $\begin{array}{l}\text { (Pourjavadi } \\
\quad \text { et al. 2014) }\end{array}$ \\
\hline 29. & $\begin{array}{l}\text { Melamine/maleic anhydride } \\
\text { (S-Me/MA) }\end{array}$ & 641.03 & Langmuir & $\begin{array}{l}\text { Pseudo } \\
\text { 2nd } \\
\text { order }\end{array}$ & Endothermic & 9.0 & $24 \mathrm{~h}$ & $0.06 \mathrm{~g}$ & $\begin{array}{l}\text { (Rong et al. } \\
\text { 2014) }\end{array}$ \\
\hline 30. & $\begin{array}{l}\text { Iron nanoparticles loaded on } \\
\text { ash (nFe-A) }\end{array}$ & 500 & Langmuir & $\begin{array}{l}\text { Pseudo } \\
\text { 2nd } \\
\text { order }\end{array}$ & Endothermic & 8.0 & $30 \mathrm{~min}$ & - & $\begin{array}{l}\text { (Agarwal et al. } \\
\text { 2016) }\end{array}$ \\
\hline 31. & $\begin{array}{l}\text { Poly(vinyl alcohol) (PVA) } \\
\text { and chitosan (CS)(PVA/CS } \\
\text { composite) }\end{array}$ & 380.65 & Freundlich & $\begin{array}{l}\text { Pseudo } \\
\text { 2nd } \\
\text { order }\end{array}$ & Endothermic & 6.0 & $540 \mathrm{~min}$ & $\begin{array}{l}0.1 \mathrm{~g} / \\
\quad 50 \mathrm{~mL}\end{array}$ & (Li et al. 2012) \\
\hline 32. & $\begin{array}{l}\text { Alginate/polyaspartate } \\
\text { composite hydrogel beads }\end{array}$ & $300-350$ & - & - & - & - & $3.0 \mathrm{~h}$ & $\begin{array}{l}0.1 \mathrm{~g} / \\
50 \mathrm{~mL}\end{array}$ & $\begin{array}{l}\text { (Jeon et al. } \\
2008)\end{array}$ \\
\hline 33. & $\begin{array}{l}\text { Polyacrylic acid (PAA)- } \\
\text { nanoclay nanocomposite }\end{array}$ & 243.11 & Freundlich & - & - & 9.2 & $15 \mathrm{~min}$ & $\begin{array}{l}0.5 \mathrm{~g} / \\
100 \mathrm{~mL}\end{array}$ & $\begin{array}{l}\text { (Sonawane et al. } \\
\text { 2009) }\end{array}$ \\
\hline 34. & $\begin{array}{l}\mathrm{Fe}_{3} \mathrm{O}_{4} @ \mathrm{PANI} \\
\text { nanocomposites }\end{array}$ & 240.00 & Langmuir & $\begin{array}{l}\text { Pseudo } \\
\text { 2nd } \\
\text { order }\end{array}$ & Exothermic & 7.0 & $4 \mathrm{~h}$ & $0.01 \mathrm{~g}$ & $\begin{array}{l}\text { (Mahto et al. } \\
\text { 2014) }\end{array}$ \\
\hline 35. & $\begin{array}{l}\text { Vinyl-modified mesoporous } \\
\text { poly }(\text { acrylic acid }) / \mathrm{SiO}_{2} \\
\left(\mathrm{PAA} / \mathrm{SiO}_{2}\right) \text { composite } \\
\text { nanofiber membranes }\end{array}$ & 220.49 & $\begin{array}{l}\text { Redlich- } \\
\quad \text { Peterson }\end{array}$ & $\begin{array}{l}\text { Pseudo } \\
\text { 2nd } \\
\text { order }\end{array}$ & - & - & $240 \mathrm{~min}$ & $\begin{array}{l}50 \mathrm{mg} / \\
50 \mathrm{~mL}\end{array}$ & (Xu et al. 2012) \\
\hline 36. & $\begin{array}{l}\text { Activated carbon/CoFe } \mathrm{O}_{4} \\
\text { composite }(\mathrm{AC} / \mathrm{CFO})\end{array}$ & 89.29 & Langmuir & $\begin{array}{l}\text { Pseudo } \\
\text { 2nd } \\
\text { order }\end{array}$ & - & 5.0 & $5 \min$ & - & (Ai et al. 2010) \\
\hline
\end{tabular}


Table 9 continued

\begin{tabular}{|c|c|c|c|c|c|c|c|c|c|}
\hline $\begin{array}{l}\text { Sr. } \\
\text { No. }\end{array}$ & Adsorbents & $\begin{array}{l}Q_{\max } \\
(\mathrm{mg} / \mathrm{g})\end{array}$ & $\begin{array}{l}\text { Isotherm } \\
\text { study }\end{array}$ & $\begin{array}{l}\text { Kinetics } \\
\text { study }\end{array}$ & $\begin{array}{l}\text { Thermodynamic } \\
\text { study }\end{array}$ & $\mathrm{pH}$ & $\begin{array}{l}\text { Equilibrium } \\
\text { time }\end{array}$ & $\begin{array}{l}\text { Dosage of } \\
\text { adsorbent }\end{array}$ & References \\
\hline 37. & $\begin{array}{l}\text { Unsaturated polyester Ce(IV) } \\
\text { phosphate (USPECe(IV)P) } \\
\text { composite }\end{array}$ & 44.85 & Freundlich & $\begin{array}{l}\text { Pseudo } \\
\text { 2nd } \\
\text { order }\end{array}$ & Endothermic & 8.0 & $30-35 \mathrm{~min}$ & $\begin{array}{l}0.1 \mathrm{~g} / \\
100 \mathrm{~mL}\end{array}$ & $\begin{array}{l}\text { (Khan et al. } \\
\text { 2013) }\end{array}$ \\
\hline 38. & $\begin{array}{l}\text { Graphene oxide } \\
\text { (GO)/cellulose bead } \\
\text { (GOCB) composites }\end{array}$ & 30.090 & Langmuir & $\begin{array}{l}\text { Pseudo } \\
\text { 2nd } \\
\text { order }\end{array}$ & Endothermic & 7.0 & $12 \mathrm{~h}$ & $0.1 \mathrm{~g}$ & $\begin{array}{l}\text { (Zhang et al. } \\
\text { 2015) }\end{array}$ \\
\hline 39. & $\begin{array}{l}\text { Carbon nanotube/polyaniline } \\
\text { composites }\end{array}$ & 15.45 & Langmuir & $\begin{array}{l}\text { Pseudo } \\
\text { 2nd } \\
\text { order }\end{array}$ & Endothermic & - & - & - & $\begin{array}{l}\text { (Zeng et al. } \\
\text { 2013) }\end{array}$ \\
\hline 40. & $\begin{array}{l}\text { MWCNTs functionalized } \\
\text { with the carboxylate group }\end{array}$ & 11.73 & Langmuir & $\begin{array}{l}\text { Pseudo } \\
\text { 2nd } \\
\text { order }\end{array}$ & Endothermic & 9.0 & $10 \mathrm{~min}$ & - & $\begin{array}{l}\text { (Rajabi et al. } \\
\text { 2016) }\end{array}$ \\
\hline 41. & $\begin{array}{l}\text { Magnetoactive } \\
\text { nanocomposite fibers }\end{array}$ & 8.809 & Langmuir & - & Endothermic & 8.0 & $24 \mathrm{~h}$ & - & $\begin{array}{l}\text { (Savva et al. } \\
\text { 2015) }\end{array}$ \\
\hline 42. & $\begin{array}{l}\text { Polyaniline-nickel ferrite } \\
\text { magnetic nanocomposite }\end{array}$ & 4.09 & Langmuir & $\begin{array}{l}\text { Pseudo } \\
\text { 2nd } \\
\text { order }\end{array}$ & - & 7.0 & $210 \mathrm{~min}$ & $5.0 \mathrm{~g} / \mathrm{L}$ & $\begin{array}{l}\text { (Patil and } \\
\text { Shrivastava } \\
\text { 2014) }\end{array}$ \\
\hline 43. & $\begin{array}{l}\text { Humic acid-immobilized } \\
\text { amine modified } \\
\text { polyacrylamide/bentonite } \\
\text { composite (HA-Am-PAA- } \\
\text { B) }\end{array}$ & $\begin{array}{l}656.50 \\
\quad(\mu \mathrm{mol} / \\
\mathrm{g})\end{array}$ & Freundlich & $\begin{array}{l}\text { Pseudo } \\
\text { 2nd } \\
\text { order }\end{array}$ & - & 8.0 & $3 \mathrm{~h}$ & $2.0 \mathrm{~g} / \mathrm{L}$ & $\begin{array}{l}\text { (Anirudhan and } \\
\text { Suchithra } \\
\text { 2009) }\end{array}$ \\
\hline 44. & $\begin{array}{l}\text { Humic acid-immobilized } \\
\text { amine-modified } \\
\text { polyacrylamide-bentonite } \\
\text { composite (HA-Am-PAA- } \\
\text { B) }\end{array}$ & $\begin{array}{l}656.50 \\
\quad(\mu \mathrm{mol} / \\
\mathrm{g})\end{array}$ & Freundlich & $\begin{array}{l}\text { Pseudo } \\
\text { 2nd } \\
\text { order }\end{array}$ & - & $5.0-8.0$ & $1 \mathrm{~h}$ & $2.0 \mathrm{~g} / \mathrm{L}$ & $\begin{array}{l}\text { (Anirudhan } \\
\text { et al. 2009) }\end{array}$ \\
\hline 45 & $\begin{array}{l}\text { Glycine-modified silica } \\
\text { nanoparticles }\end{array}$ & - & Langmuir & $\begin{array}{l}\text { Pseudo } \\
\text { 1st } \\
\text { order }\end{array}$ & - & 10.0 & $40 \mathrm{~min}$ & - & $\begin{array}{l}\text { (Mansa et al. } \\
\text { 2016) }\end{array}$ \\
\hline 46. & $\begin{array}{l}\text { Magnetic Pycnoporus } \\
\text { sanguineus-Loaded } \\
\text { Alginate Composite Beads }\end{array}$ & - & - & - & - & - & - & - & $\begin{array}{l}\text { (Yang et al. } \\
\text { 2014) }\end{array}$ \\
\hline 47. & $\begin{array}{l}\text { Polyaniline/ZnO } \\
\text { nanocomposite }\end{array}$ & - & - & - & - & - & $5 \mathrm{~h}$ & $0.4 \mathrm{~g} / \mathrm{L}$ & $\begin{array}{l}\text { (Eskizeybek } \\
\text { et al. 2012) }\end{array}$ \\
\hline 48. & $\begin{array}{l}\mathrm{Fe}_{0.01} \mathrm{Ni}_{0.01} \mathrm{Zn}_{0.98} \mathrm{O} / \\
\text { polyacrylamide } \\
\text { nanocomposite }\end{array}$ & - & - & - & - & - & - & - & $\begin{array}{l}\text { (Kant et al. } \\
\text { 2014) }\end{array}$ \\
\hline 49. & $\begin{array}{l}\text { Hierarchically macro/micro- } \\
\text { porous silicon-carbon- } \\
\text { nitrogen }(\mathrm{Si}-\mathrm{C}-\mathrm{N}) \text { hybrid } \\
\text { material }\end{array}$ & - & - & - & - & - & - & - & $\begin{array}{l}\text { (Meng et al. } \\
\text { 2015) }\end{array}$ \\
\hline
\end{tabular}

adsorption equilibrium and kinetic data was well fitted and found to be in good agreement with the Langmuir isotherm model and pseudo second order kinetic model, respectively.

Shirmardi et al. (2013) prepared functionalized multiwalled carbon nanotubes ( $f$-MWCNTs) by acid treatment and used for the adsorption of $\mathrm{MG}$ dye from aqueous solution in batch mode. The results indicated that by increasing contact time, $\mathrm{pH}$ and adsorbent dosage, the removal percentage increased, but by increasing the initial
MG concentration, the removal percentage decreased. Based on correlation coefficient $\left(R^{2}\right)$, the experimental data follows the Langmuir isotherm with maximum adsorption capacity of $142.85 \mathrm{mg} / \mathrm{g}$.

Halloysite, a mineral of the kaolin group [forms hollow tubular crystals (halloysite nanotubes-HNTs)], can be used as MG dye adsorbent by Kiani et al. (2011) and found that the equilibrium adsorption was reached within $30 \mathrm{~min}$. The adsorption of MG by HNTs obeyed pseudo-secondorder kinetics with activation energy $18.28 \mathrm{~kJ} / \mathrm{mol}$, 
suggested that the process was physisorption. The best-fit adsorption isotherm was achieved with the Langmuir model, indicating that homogeneous adsorption occurred. The negative values of $\Delta G^{\circ}$ and positive value of $\Delta H^{\circ}$ showed that the adsorption was a spontaneous and endothermic.

The particles of at least one dimension smaller than $1 \mu \mathrm{m}$, and potentially as small as atomic and molecular length scales $(0.2 \mathrm{~nm})$ are called nanoparticles. Nanoparticles can have amorphous or crystalline form (Buzea et al. 2007). Due to some of the unique characteristics such as small size, catalytic potential, large surface area, ease of separation, and large number of active sites for interaction with different contaminants the nanoparticles have established themselves as excellent adsorbents (Raval et al. 2016d).

\section{Composites and nano-composites}

The nano-scale inclusions that are imbedded within matrix of a material are referred as composites (Chen et al. 2013).

Poly(vinyl alcohol) (PVA) is a water-soluble material containing many reactive hydroxyl groups. It has been extensively applied in biomedical and pharmaceutical fields due to its low cost, non-toxicity, biocompatibility, good mechanical strength and chemical stability. However, the PVA foam illustrated low adsorption for organic and inorganic pollutants. Chitosan (CS) is one of the most abundant biopolymers in nature. It exhibits good adsorption capacity for organic dyes and heavy metals because it has a large number of hydroxyl and amine groups.
However, the use of CS in the form of flake or powder cannot be separated easily. Therefore, Li et al. (2012) prepared a novel foam adsorbent based on PVA/CS composites by the interpenetrating polymer network (IPN), foaming process and freeze-thaw treatment and found that the composite material had higher adsorption efficiency (380.65 mg/g) compared with the single component.

Hussain et al. (2006) defined nanocomposites as materials consisting of at least two phases with one dispersed in another that is called matrix, and thus forms a three dimensional network.

Wang et al. (2015) prepared Magnetic $\beta$-cyclodextringraphene oxide nanocomposites $\left(\mathrm{Fe}_{3} \mathrm{O}_{4} / \beta-\mathrm{CD} / \mathrm{GO}\right)$ as an adsorbent for $\mathrm{MG}$ dye because graphene oxide have abundant hydrophilic groups on its surface, and thus it has good adsorption capacity still its separation was difficult task from sample solution and hence, to simplify the retrieval procedure, magnetic adsorbent $\left(\mathrm{Fe}_{3} \mathrm{O}_{4}\right)$ has been incorporated with GO. In addition, $\beta-\mathrm{CD}$ was also added onto composite (Magnetic-graphene oxide) to provide hydrophobic inner cavity and a hydrophilic exterior which can enhance the adsorption capacity of composite adsorbent for MG dye.

Table 9 presented the maximum adsorption capacities of various nanomaterials, composite and nanocomposite adsorbents used for MG.

\section{Miscellaneous adsorbents}

See Table 10.

Table 10 The maximum adsorption capacities and other optimal conditions of various miscellaneous adsorbents for the removal of MG from water and wastewater

\begin{tabular}{|c|c|c|c|c|c|c|c|c|c|}
\hline $\begin{array}{l}\text { Sr. } \\
\text { No. }\end{array}$ & Adsorbents & $\begin{array}{l}Q_{\max } \\
(\mathrm{mg} / \mathrm{g})\end{array}$ & $\begin{array}{l}\text { Isotherm } \\
\text { study }\end{array}$ & $\begin{array}{l}\text { Kinetics } \\
\text { study }\end{array}$ & $\begin{array}{l}\text { Thermodynamic } \\
\text { study }\end{array}$ & $\mathrm{pH}$ & $\begin{array}{l}\text { Equilibrium } \\
\text { time }\end{array}$ & $\begin{array}{l}\text { Dosage of } \\
\text { adsorbent }\end{array}$ & References \\
\hline 1. & $\begin{array}{l}\text { Zeolitic imidazole framework-67 } \\
\text { (ZIF-67) }\end{array}$ & 3227.00 & Langmuir & $\begin{array}{l}\text { Pseudo } \\
\text { 2nd } \\
\text { order }\end{array}$ & Endothermic & - & $2 \mathrm{~h}$ & $0.25 \mathrm{~g} / \mathrm{L}$ & $\begin{array}{l}\text { (Lin and } \\
\text { Chang } \\
\text { 2015) }\end{array}$ \\
\hline 2. & Graphite oxide (GO) & 248.00 & Langmuir & $\begin{array}{l}\text { Pseudo } \\
\text { 2nd } \\
\text { order }\end{array}$ & - & - & $20 \min$ & $\begin{array}{l}10 \mathrm{mg} / \\
500 \mathrm{~mL}\end{array}$ & $\begin{array}{l}\text { (Bradder } \\
\quad \text { et al. 2011) }\end{array}$ \\
\hline 3. & Poly- $\gamma$-glutamic acid ( $\gamma$-PGA) & 293.32 & $\begin{array}{l}\text { Redlich- } \\
\text { Peterson }\end{array}$ & $\begin{array}{l}\text { Pseudo } \\
\text { 2nd } \\
\text { order }\end{array}$ & & $>5.0$ & $2 \mathrm{~h}$ & $0.4 \mathrm{~g} / \mathrm{L}$ & $\begin{array}{l}\text { (Stephen } \\
\text { Inbaraj } \\
\text { et al. 2006) }\end{array}$ \\
\hline 4. & Defective coffee beans press cake & 208.30 & Langmuir & $\begin{array}{l}\text { Pseudo } \\
\text { 2nd } \\
\text { order }\end{array}$ & - & 5.0 & $4.0 \mathrm{~h}$ & $5.0 \mathrm{~g} / \mathrm{L}$ & $\begin{array}{l}\text { (Franca et al. } \\
\text { 2010a) }\end{array}$ \\
\hline 5. & $\begin{array}{l}\text { Polyethylene terephthalate (PET) by } \\
\text { chemical activation with } \mathrm{NaOH} \\
\text { (PETNa8) }\end{array}$ & 169.49 & Langmuir & $\begin{array}{l}\text { Pseudo } \\
1 \text { st } \\
\text { order }\end{array}$ & - & 10.0 & $90 \min$ & $\begin{array}{l}0.1 \mathrm{~g} / \\
25 \mathrm{~mL}\end{array}$ & $\begin{array}{r}\text { (Akmil-Başar } \\
\text { et al. 2005) }\end{array}$ \\
\hline 6. & Magnetic sorbent ("blue magnetite") & 93.20 & Langmuir & - & - & - & $30 \mathrm{~min}$ & - & $\begin{array}{l}\text { (Šafařík et al. } \\
1995)\end{array}$ \\
\hline
\end{tabular}


Table 10 continued

\begin{tabular}{|c|c|c|c|c|c|c|c|c|c|}
\hline $\begin{array}{l}\text { Sr. } \\
\text { No. }\end{array}$ & Adsorbents & $\begin{array}{l}Q_{\max } \\
(\mathrm{mg} / \mathrm{g})\end{array}$ & $\begin{array}{l}\text { Isotherm } \\
\text { study }\end{array}$ & $\begin{array}{l}\text { Kinetics } \\
\text { study }\end{array}$ & $\begin{array}{l}\text { Thermodynamic } \\
\text { study }\end{array}$ & $\mathrm{pH}$ & $\begin{array}{l}\text { Equilibrium } \\
\text { time }\end{array}$ & $\begin{array}{l}\text { Dosage of } \\
\text { adsorbent }\end{array}$ & References \\
\hline 7. & Polymeric gel & 4.900 & Langmuir & $\begin{array}{l}\text { Pseudo } \\
\text { 2nd } \\
\text { order }\end{array}$ & Exothermic & - & - & - & $\begin{array}{l}\text { (Malana et al. } \\
\text { 2010) }\end{array}$ \\
\hline 8. & $\begin{array}{l}\text { Polylactide (PLA)/spent brewery } \\
\text { grains (SBGs) films }\end{array}$ & 1.484 & Freundlich & $\begin{array}{l}\text { Pseudo } \\
\text { 2nd } \\
\text { order }\end{array}$ & - & 6.89 & $95 \min$ & $0.42 \mathrm{~g}$ & $\begin{array}{l}\text { (Chanzu et al. } \\
\text { 2012) }\end{array}$ \\
\hline 9. & Polyethylene glycol (PEG) micelles & - & Langmuir & - & Endothermic & - & $10 \min$ & - & $\begin{array}{l}\text { (Chen et al. } \\
\text { 2009) }\end{array}$ \\
\hline 10. & polystyrene microspheres & - & - & - & - & - & - & - & $\begin{array}{l}\text { (Eckenrode } \\
\quad \text { et al. 2005) }\end{array}$ \\
\hline 11. & $\begin{array}{l}\text { Magnetic affinity adsorbent (blue } \\
\text { magnetite) }\end{array}$ & - & - & - & - & - & - & - & $\begin{array}{l}\text { (Šafařík and } \\
\text { Šafaříková } \\
\text { 2002) }\end{array}$ \\
\hline 12. & $\begin{array}{l}\text { Loosely bound extracellular } \\
\text { polymeric substances (LB-EPS), } \\
\text { tightly bound EPS (TB-EPS) }\end{array}$ & - & - & - & - & 7.0 & - & - & $\begin{array}{l}\text { (Gao et al. } \\
\text { 2011) }\end{array}$ \\
\hline 13. & Amberlite XAD-4 resin & - & - & $\begin{array}{l}\text { Pseudo } \\
\text { 1st } \\
\text { order }\end{array}$ & Endothermic & - & $150 \mathrm{~min}$ & - & $\begin{array}{l}\text { (Dos Reis } \\
\text { et al. 2011) }\end{array}$ \\
\hline 14. & AmberliteXAD-2 resin & - & - & $\begin{array}{l}\text { Pseudo } \\
\text { 1st } \\
\text { order }\end{array}$ & Endothermic & - & $150 \mathrm{~min}$ & - & $\begin{array}{l}\text { (Dos Reis } \\
\text { et al. 2011) }\end{array}$ \\
\hline 15. & $\begin{array}{l}\text { Metal-organic framework MIL- } \\
100(\mathrm{Fe})\end{array}$ & - & Freundlich & $\begin{array}{l}\text { Pseudo } \\
\text { 2nd } \\
\text { order }\end{array}$ & Endothermic & 5.0 & - & - & $\begin{array}{l}\text { (Huo and Yan } \\
\text { 2012) }\end{array}$ \\
\hline 16. & D201_Lac & - & - & - & - & - & - & - & $\begin{array}{l}\text { (Zhang et al. } \\
\text { 2012) }\end{array}$ \\
\hline 17. & Mesoporous $\mathrm{Cu} / \mathrm{Al}_{2} \mathrm{O}_{3}-\mathrm{MCM}-41$ & - & - & - & - & 11.0 & $30 \mathrm{~min}$ & $1.0 \mathrm{~g} / \mathrm{L}$ & $\begin{array}{l}\text { (Pradhan } \\
\quad \text { et al. 2011) }\end{array}$ \\
\hline 18. & $\begin{array}{l}\text { Calcium oxalate-bromopyrogallol } \\
\text { red inclusion sorbent }\end{array}$ & - & - & - & - & - & - & - & $\begin{array}{l}\text { (Wang and } \\
\text { Gao 2009) }\end{array}$ \\
\hline 19. & $\begin{array}{l}\text { Polyurethane foam (PUF) loaded with } \\
\text { sodium dodecylsulfate (SDS) }\end{array}$ & - & - & - & - & - & - & - & $\begin{array}{l}\text { (Robaina } \\
\quad \text { et al. 2009) }\end{array}$ \\
\hline 20. & $\begin{array}{l}\text { Functionalized magnetic adsorbents } \\
\text { (FMAs) }\end{array}$ & - & - & - & - & - & - & - & $\begin{array}{l}\text { (Wang et al. } \\
\text { 2014b) }\end{array}$ \\
\hline
\end{tabular}

\section{Conclusions and future perspectives}

Adsorption process being very easy, economical, effective and versatile had become the most favored methods for amputation of toxic and hazardous dyes from wastewater. This review article revealed about various adsorbents, such as activated carbons, low-cost materials, nanomaterials, composites and nanocomposites, used for the adsorption and removal of MG from water and wastewater. It was concluded that amongst all the other adsorbents, agricultural solid wastes and biosorbents, such as biopolymers and biomass, have been extensively used for the removal of MG and are acknowledged as effective and economic adsorbents for the removal of MG dye. Various experimental conditions $(\mathrm{pH}$, temperature, contact time and amount of adsorbent) have been also mentioned for related adsorbents in the present article.

This review article also disclosed that the Langmuir and Freundlich adsorption isotherm models were frequently applied to evaluate the adsorption capacity of various adsorbents towards MG. In kinetic study, it was observed that the pseudo-second-order rate model was been followed for the adsorption of $\mathrm{MG}$ and according to thermodynamic parameters the adsorption process was found to be spontaneous in nature.

Since, most of the reported studies had been performed using batch and small-scale column adsorption tests, further research is required for the development of more effective adsorbents, modeling of adsorption mechanism, regeneration of spent adsorbents and treatment of real industrial wastewater. 
Acknowledgements The authors gratefully acknowledge the financial assistance provided by the INSPIRE Programme under the Assured Opportunity for Research Careers (AORC) scheme, funded by the Department of Science and Technology (DST) (Sanction Order No.: DST/INSPIRE Fellowship/2013/66).

Open Access This article is distributed under the terms of the Creative Commons Attribution 4.0 International License (http:// creativecommons.org/licenses/by/4.0/), which permits unrestricted use, distribution, and reproduction in any medium, provided you give appropriate credit to the original author(s) and the source, provide a link to the Creative Commons license, and indicate if changes were made.

\section{References}

Abd-El-Kareem MS, Taha HM (2012) Decolorization of malachite green and methylene blue by two microalgal species. Int J Chem Environ Eng 3:297-302

Adegoke KA, Bello OS (2015) Dye sequestration using agricultural wastes as adsorbents. Water Resour Ind 12:8-24. doi:10.1016/j. wri.2015.09.002

Afkhami A, Moosavi R, Madrakian T (2010) Preconcentration and spectrophotometric determination of low concentrations of malachite green and leuco-malachite green in water samples by high performance solid phase extraction using maghemite nanoparticles. Talanta 82:785-789. doi:10.1016/j.talanta.2010. 05.054

Agarwal S, Tyagi I, Gupta VK et al (2016) Kinetics and thermodynamics of Malachite Green dye removal from aqueous phase using iron nanoparticles loaded on ash. J Mol Liq. doi:10.1016/j. molliq.2016.04.039

Ahmad MA, Ahmad N, Bello OS (2014) Adsorptive removal of malachite green dye using durian seed-based activated carbon. Water Air Soil Pollut 225:1-18. doi:10.1007/s11270-014-2057-Z

Ahmad MA, Alrozi R (2011) Removal of malachite green dye from aqueous solution using rambutan peel-based activated carbon: equilibrium, kinetic and thermodynamic studies. Chem Eng $\mathrm{J}$ 171:510-516. doi:10.1016/j.cej.2011.04.018

Ahmad R, Kumar R (2010) Adsorption studies of hazardous malachite green onto treated ginger waste. J Environ Manag 91:1032-1038. doi:10.1016/j.jenvman.2009.12.016

Ahmad R, Mondal PK (2010) Application of modified water nut carbon as a sorbent in congo red and malachite green dye contaminated wastewater remediation. Sep Sci Technol 45:394-403. doi:10.1080/01496390903484875

Ai L, Huang H, Chen $\mathrm{Z}$ et al (2010) Activated carbon/CoFe2O4 composites: facile synthesis, magnetic performance and their potential application for the removal of malachite green from water. Chem Eng J 156:243-249. doi:10.1016/j.cej.2009.08.028

Aitcheson SJ, Arnett J, Murray KR, Zhang J (2000) Removal of aquaculture therapeutants by carbon adsorption: 1. Equilibrium adsorption behaviour of single components. Aquaculture 183:269-284. doi:10.1016/S0044-8486(99)00304-X

Akar E, Altinişik A, Seki Y (2013) Using of activated carbon produced from spent tea leaves for the removal of malachite green from aqueous solution. Ecol Eng 52:19-27. doi:10.1016/j. ecoleng.2012.12.032

Akmil-Başar C, Onal Y, Kiliçer T, Eren D (2005) Adsorptions of high concentration malachite green by two activated carbons having different porous structures. J Hazard Mater 127:73-80. doi:10. 1016/j.jhazmat.2005.06.025

Aksakal O, Ucun H, Kaya Y (2009) Application of Eriobotrya japonica (Thunb.) Lindley (Loquat) seed biomass as a new biosorbent for the removal of malachite green from aqueous solution. Water Sci Technol J Int Assoc Water Pollut Res 59:1631-1639. doi:10.2166/wst.2009.130

Ali H (2010) Biodegradation of synthetic dyes-a review. Water Air Soil Pollut 213:251-273. doi:10.1007/s11270-010-0382-4

Ali H, Ahmad W, Haq T (2009) Decolorization and degradation of malachite green by Aspergillus flavus and Alternaria solani. Afr J Biotechnol. doi:10.4314/ajb.v8i8.60239

Aliyan H, Fazaeli R, Jalilian R (2013) Fe3O4@mesoporous SBA-15: a magnetically recoverable catalyst for photodegradation of malachite green. Appl Surf Sci 276:147-153. doi:10.1016/j. apsusc.2013.03.049

Altınışık A, Gür E, Seki Y (2010) A natural sorbent, Luffa cylindrica for the removal of a model basic dye. J Hazard Mater 179:658-664. doi:10.1016/j.jhazmat.2010.03.053

An S-Y, Min S-K, Cha I-H et al (2002) Decolorization of triphenylmethane and azo dyes by Citrobacter sp. Biotechnol Lett 24:1037-1040. doi:10.1023/A:1015610018103

Anastopoulos I, Kyzas GZ (2014) Agricultural peels for dye adsorption: a review of recent literature. J Mol Liq 200(Part B):381-389. doi:10.1016/j.molliq.2014.11.006

Anbia M, Ghaffari A (2012) Removal of malachite green from dye wastewater using mesoporous carbon adsorbent. J Iran Chem Soc 8:S67-S76. doi:10.1007/BF03254283

Anirudhan TS, Suchithra PS (2009) Adsorption characteristics of humic acid-immobilized amine modified polyacrylamide/bentonite composite for cationic dyes in aqueous solutions. J Environ Sci China 21:884-891

Anirudhan TS, Suchithra PS, Radhakrishnan PG (2009) Synthesis and characterization of humic acid immobilized-polymer/bentonite composites and their ability to adsorb basic dyes from aqueous solutions. Appl Clay Sci 43:336-342. doi:10.1016/j.clay.2008. 09.015

Anjaneyulu Y, Chary NS, Raj DSS (2005) Decolourization of industrial effluents-available methods and emerging technologies-a review. Rev Environ Sci Biotechnol 4:245-273. doi:10. 1007/s11157-005-1246-z

Arellano-Cárdenas S, López-Cortez S, Cornejo-Mazón M, MaresGutiérrez JC (2013) Study of malachite green adsorption by organically modified clay using a batch method. Appl Surf Sci 280:74-78. doi:10.1016/j.apsusc.2013.04.097

Arivoli S, Sundaravadivelu M, Elango KP (2008) Removal of basic and acidic dyes from aqueous solution by adsorption on a low cost activated carbon: kinetic and thermodynamic study. Indian $\mathbf{J}$ Chem Technol 15:130

Asiltürk M, Sayılkan F, Arpaç E (2009) Effect of Fe3+ ion doping to $\mathrm{TiO} 2$ on the photocatalytic degradation of malachite green dye under UV and vis-irradiation. J Photochem Photobiol Chem 203:64-71. doi:10.1016/j.jphotochem.2008.12.021

Ayed L, Chaieb K, Cheref A, Bakhrouf A (2008) Biodegradation of triphenylmethane dye Malachite Green by Sphingomonas paucimobilis. World J Microbiol Biotechnol 25:705-711. doi:10.1007/ s11274-008-9941-x

Ayed L, Chaieb K, Cheref A, Bakhrouf A (2010) Biodegradation and decolorization of triphenylmethane dyes by Staphylococcus epidermidis. Desalination 260:137-146. doi:10.1016/j.desal. 2010.04.052

Babel S, Kurniawan TA (2003) Low-cost adsorbents for heavy metals uptake from contaminated water: a review. J Hazard Mater 97:219-243. doi:10.1016/S0304-3894(02)00263-7

Baek M-H, Ijagbemi CO, Se-Jin O, Kim D-S (2010) Removal of malachite green from aqueous solution using degreased coffee bean. J Hazard Mater 176:820-828. doi:10.1016/j.jhazmat.2009. 11.110

Bai C, Xiao W, Feng D et al (2013) Efficient decolorization of Malachite Green in the Fenton reaction catalyzed by [Fe(III)-

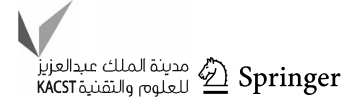


salen]Cl complex. Chem Eng J 215-216:227-234. doi:10.1016/j. cej.2012.09.124

Banat IM, Nigam P, Singh D, Marchant R (1996) Microbial decolorization of textile-dye containing effluents: a review. Bioresour Technol 58:217-227. doi:10.1016/S09608524(96)00113-7

Bansal P, Bhullar N, Sud D (2009) Studies on photodegradation of malachite green using $\mathrm{TiO}_{2} / \mathrm{ZnO}$ photocatalyst. Desalin Water Treat 12:108-113. doi:10.5004/dwt.2009.944

Başar CA (2006) Applicability of the various adsorption models of three dyes adsorption onto activated carbon prepared waste apricot. J Hazard Mater 135:232-241. doi:10.1016/j.jhazmat. 2005.11.055

Beak MH, Ijagbemi CO, Kim DS (2009) Treatment of malachite green-containing wastewater using poultry feathers as adsorbent. J Environ Sci Health Part A Tox Hazard Subst Environ Eng 44:536-542. doi:10.1080/10934520902720132

Bejarano-Pérez NJ, Suárez-Herrera MF (2008) Sonochemical and sonophotocatalytic degradation of malachite green: the effect of carbon tetrachloride on reaction rates. Ultrason Sonochem 15:612-617. doi:10.1016/j.ultsonch.2007.09.009

Bekçi Z, Özveri C, Seki Y, Yurdakoç K (2008) Sorption of malachite green on chitosan bead. J Hazard Mater 154:254-261. doi:10. 1016/j.jhazmat.2007.10.021

Bekçi Z, Seki Y, Cavas L (2009) Removal of malachite green by using an invasive marine alga Caulerpa racemosa var. cylindracea. J Hazard Mater 161:1454-1460. doi:10.1016/j.jhazmat. 2008.04.125

Bello OS, Ahmad MA, Ahmad N (2012) Adsorptive features of banana (Musa paradisiaca) stalk-based activated carbon for malachite green dye removal. Chem Ecol 28:153-167. doi:10. 1080/02757540.2011.628318

Bello OS, Ahmad MA, Semire B (2014) Scavenging malachite green dye from aqueous solutions using pomelo (Citrus grandis) peels: kinetic, equilibrium and thermodynamic studies. Desalin Water Treat 56:521-535. doi:10.1080/19443994.2014.940387

Bello OS, Bello IA, Adegoke KA (2013) Adsorption of dyes using different types of sand: a review. South Afr J Chem 66:117-129

Berberidou C, Poulios I, Xekoukoulotakis NP, Mantzavinos D (2007) Sonolytic, photocatalytic and sonophotocatalytic degradation of malachite green in aqueous solutions. Appl Catal B Environ 74:63-72. doi:10.1016/j.apcatb.2007.01.013

Bhagavathi Pushpa T, Vijayaraghavan J, Sardhar Basha SJ et al (2015) Investigation on removal of malachite green using EM based compost as adsorbent. Ecotoxicol Environ Saf 118:177-182. doi:10.1016/j.ecoenv.2015.04.033

Bhattacharyya KG, Sarma A (2003) Adsorption characteristics of the dye, brilliant green, on neem leaf powder. Dyes Pigments 57:211-222. doi:10.1016/S0143-7208(03)00009-3

Bojinova A, Dushkin C (2011) Photodegradation of malachite green in water solutions by means of thin films of $\mathrm{TiO}_{2} / \mathrm{WO}_{3}$ under visible light. React Kinet Mech Catal 103:239-250. doi:10.1007/ s11144-011-0295-2

Boutemedjet S, Hamdaoui O (2009) Sorption of malachite green by eucalyptus bark as a non-conventional low-cost biosorbent. Desalin Water Treat 8:201-210. doi:10.5004/dwt.2009.684

Bradder P, Ling SK, Wang S, Liu S (2011) Dye adsorption on layered graphite oxide. J Chem Eng Data 56:138-141. doi:10.1021/ je101049g

Breck DW (1984) Zeolite molecular sieves. Krieger, Chicago

Bulut E, Özacar M, Şengil İA (2008) Equilibrium and kinetic data and process design for adsorption of Congo Red onto bentonite. J Hazard Mater 154:613-622. doi:10.1016/j.jhazmat.2007.10. 071

Burkinshaw SM (2016) Physico-chemical aspects of textile coloration, 1st edn. Wiley, New York
Buvaneswari N, Kannan C (2011) Plant toxic and non-toxic nature of organic dyes through adsorption mechanism on cellulose surface. J Hazard Mater 189:294-300. doi:10.1016/j.jhazmat. 2011.02.036

Buzea C, Pacheco II, Robbie K (2007) Nanomaterials and nanoparticles: sources and toxicity. Biointerphases 2:MR17-MR71. doi: $10.1116 / 1.2815690$

Cardon D (2007) Natural dyes: sources, tradition, technology and science. Archetype, London

Chanzu HA, Onyari JM, Shiundu PM (2012) Biosorption of malachite green from aqueous solutions onto polylactide/spent brewery grains films: kinetic and equilibrium studies. J Polym Environ 20:665-672. doi:10.1007/s10924-012-0479-5

Cheknane B, Baudu M, Basly J-P, Bouras O (2010) Adsorption of basic dyes in single and mixture systems on granular inorganicorganic pillared clays. Environ Technol 31:815-822. doi:10. 1080/09593331003667758

Chen CC, Lu CS, Chung YC, Jan JL (2007) UV light induced photodegradation of malachite green on $\mathrm{TiO} 2$ nanoparticles. J Hazard Mater 141:520-528. doi:10.1016/j.jhazmat.2006.07. 011

Chen F, Ma W, He J, Zhao J (2002) Fenton degradation of malachite green catalyzed by aromatic additives. J Phys Chem A 106:9485-9490. doi:10.1021/jp0144350

Chen J, Mao J, Mo X et al (2009) Study of adsorption behavior of malachite green on polyethylene glycol micelles in cloud point extraction procedure. Colloids Surf Physicochem Eng Asp 345:231-236. doi:10.1016/j.colsurfa.2009.05.015

Chen Y, Zhang Y, Liu C et al (2011) Photodegradation of malachite green by nanostructured Bi2WO6 visible light-induced photocatalyst. Int J Photoenergy 2012:e510158. doi:10.1155/2012/ 510158

Chen Z, Deng H, Chen C et al (2014) Biosorption of malachite green from aqueous solutions by Pleurotus ostreatus using Taguchi method. J Environ Health Sci Eng 12:63. doi:10.1186/2052$336 \mathrm{X}-12-63$

Chen Z-Y, Gao H-W, He Y-Y (2013) Selective photodegradation and backfilling for regeneration of the inorganic-organic hybrid composite Fe3O4@C18ADB@Zn2SiO4 which captures organic pollutants from aqueous solution. RSC Adv 3:5815-5818. doi:10.1039/C3RA22324H

Cheng W, Wang S-G, Lu L et al (2008) Removal of malachite green (MG) from aqueous solutions by native and heat-treated anaerobic granular sludge. Biochem Eng J 39:538-546. doi:10. 1016/j.bej.2007.10.016

Cheriaa J, Bakhrouf A (2009) Triphenylmethanes, malachite green and crystal violet dyes decolourisation by Sphingomonas paucimobilis. Ann Microbiol 59:57-61. doi:10.1007/BF03175599

Chieng HI, Lim LBL, Priyantha N (2015) Enhancing adsorption capacity of toxic malachite green dye through chemically modified breadnut peel: equilibrium, thermodynamics, kinetics and regeneration studies. Environ Technol 36:86-97. doi:10. 1080/09593330.2014.938124

Chieng HI, Zehra T, Lim LBL et al (2014) Sorption characteristics of peat of Brunei Darussalam IV: equilibrium, thermodynamics and kinetics of adsorption of methylene blue and malachite green dyes from aqueous solution. Environ Earth Sci 72:2263-2277. doi:10.1007/s12665-014-3135-7

Chowdhury S, Chakraborty S, Saha P (2011a) Biosorption of Basic Green 4 from aqueous solution by Ananas comosus (pineapple) leaf powder. Colloids Surf B Biointerfaces 84:520-527. doi:10. 1016/j.colsurfb.2011.02.009

Chowdhury S, Das P (2012) Utilization of a domestic wasteeggshells for removal of hazardous malachite green from aqueous solutions. Environ Prog Sustain Energy 31:415-425. doi:10.1002/ep.10564 
Chowdhury S, Mishra R, Saha P, Kushwaha P (2011b) Adsorption thermodynamics, kinetics and isosteric heat of adsorption of malachite green onto chemically modified rice husk. Desalination 265:159-168. doi:10.1016/j.desal.2010.07.047

Chowdhury S, Saha P (2010a) Sea shell powder as a new adsorbent to remove Basic Green 4 (malachite green) from aqueous solutions: equilibrium, kinetic and thermodynamic studies. Chem Eng J 164:168-177. doi:10.1016/j.cej.2010.08.050

Chowdhury S, Saha P (2010b) Pseudo-second-order kinetic model for sorption of malachite green onto sea shell: comparison of linear and non-linear methods. IIOAB J 1:3-7

Chowdhury S, Saha P (2011a) Adsorption thermodynamics and kinetics of malachite green onto $\mathrm{Ca}(\mathrm{OH})_{2}$-treated fly ash. J Environ Eng 137:388-397. doi:10.1061/(ASCE)EE.19437870.0000334

Chowdhury S, Saha PD (2011) Mechanistic, Kinetic, and Thermodynamic Evaluation of Adsorption of Hazardous Malachite Green onto Conch Shell Powder. Sep Sci Technol 46:1966-1976. doi:10.1080/01496395.2011.584930

Chowdhury S, Saha PD (2012) Fixed-bed adsorption of malachite green onto binary solid mixture of adsorbents: seashells and eggshells. Toxicol Environ Chem 94:1272-1282. doi:10.1080/ 02772248.2012 .703205

Chowdhury S, Saha PD (2013) Adsorption of malachite green from aqueous solution by naoh-modified rice husk: fixed-bed column studies. Environ Prog Sustain Energy 32:633-639. doi:10.1002/ ep.11674

Chowdhury S, Saha PD (2011b) Comparative analysis of linear and nonlinear methods of estimating the pseudo-second-order kinetic parameters for sorption of malachite green onto pretreated rice husk. Bioremediat J 15:181-188. doi:10.1080/10889868.2011. 624140

Chowdhury S, Saha PD, Ghosh U (2012) Fish (Labeo rohita) scales as potential low-cost biosorbent for removal of malachite green from aqueous solutions. Bioremediat J 16:235-242. doi:10.1080/ 10889868.2012 .731444

Crini G (2006) Non-conventional low-cost adsorbents for dye removal: a review. Bioresour Technol 97:1061-1085. doi:10. 1016/j.biortech.2005.05.001

Crini G, Peindy HN, Gimbert F, Robert C (2007) Removal of C.I. Basic Green 4 (malachite green) from aqueous solutions by adsorption using cyclodextrin-based adsorbent: kinetic and equilibrium studies. Sep Purif Technol 53:97-110. doi:10. 1016/j.seppur.2006.06.018

da Fonseca MG, de Oliveira MM, Arakaki LNH (2006) Removal of cadmium, zinc, manganese and chromium cations from aqueous solution by a clay mineral. J Hazard Mater 137:288-292. doi:10. 1016/j.jhazmat.2006.02.001

Dadhaniya PV, Patel MP, Patel RG (2006) Swelling and dye adsorption study of novel superswelling [acrylamide/Nvinylpyrrolidone/3(2-hydroxyethyl carbamoyl) acrylic acid] hydrogels. Polym Bull 57:21-31. doi:10.1007/s00289-0060531-5

Dahri MK, Kooh MRR, Lim LBL (2014) Water remediation using low cost adsorbent walnut shell for removal of malachite green: equilibrium, kinetics, thermodynamic and regeneration studies. J Environ Chem Eng 2:1434-1444. doi:10.1016/j.jece.2014.07. 008

Daneshvar N, Ayazloo M, Khataee AR, Pourhassan M (2007a) Biological decolorization of dye solution containing malachite green by microalgae Cosmarium sp. Bioresour Technol 98:1176-1182. doi:10.1016/j.biortech.2006.05.025

Daneshvar N, Khataee AR, Rasoulifard MH, Pourhassan M (2007b) Biodegradation of dye solution containing malachite green: optimization of effective parameters using Taguchi method.
J Hazard Mater 143:214-219. doi:10.1016/j.jhazmat.2006.09. 016

Das A, Pal A, Saha S, Maji SK (2009a) Behaviour of fixed-bed column for the adsorption of malachite green on surfactantmodified alumina. J Environ Sci Health Part A Tox Hazard Subst Environ Eng 44:265-272. doi:10.1080/10934520802597929

Das AK, Saha S, Pal A, Maji SK (2009b) Surfactant-modified alumina: an efficient adsorbent for malachite green removal from water environment. J Environ Sci Health Part A 44:896-905. doi:10.1080/10934520902958708

Dawood S, Sen TK (2014) Review on dye removal from Its aqueous solution into alternative cost effective and non-conventional adsorbents. J Chem Process Eng 1:1-7

Debrassi A, Corrêa AF, Baccarin T et al (2012) Removal of cationic dyes from aqueous solutions using $\mathrm{N}$-benzyl- $O$-carboxymethylchitosan magnetic nanoparticles. Chem Eng J 183:284-293. doi:10.1016/j.cej.2011.12.078

Demirbas A (2009) Agricultural based activated carbons for the removal of dyes from aqueous solutions: a review. J Hazard Mater 167:1-9. doi:10.1016/j.jhazmat.2008.12.114

Dhodapkar R, Rao NN, Pande SP et al (2007) Adsorption of cationic dyes on Jalshakti ${ }^{\circledR}$, super absorbent polymer and photocatalytic regeneration of the adsorbent. React Funct Polym 67:540-548. doi:10.1016/j.reactfunctpolym.2007.03.007

Dos Reis LGT, Robaina NF, Pacheco WF, Cassella RJ (2011) Separation of malachite green and methyl green cationic dyes from aqueous medium by adsorption on Amberlite XAD-2 and XAD-4 resins using sodium dodecylsulfate as carrier. Chem Eng J 171:532-540. doi:10.1016/j.cej.2011.04.024

Du L-N, Wang S, Li G et al (2011) Biodegradation of malachite green by Pseudomonas sp. strain DY1 under aerobic condition: characteristics, degradation products, enzyme analysis and phytotoxicity. Ecotoxicology 20:438-446. doi:10.1007/s10646011-0595-3

Eckenrode HM, Jen S-H, Han J et al (2005) Adsorption of a cationic dye molecule on polystyrene microspheres in colloids: effect of surface charge and composition probed by second harmonic generation. J Phys Chem B 109:4646-4653. doi:10.1021/ jp045610q

Eichlerová I, Homolka L, Nerud F (2006) Synthetic dye decolorization capacity of white rot fungus Dichomitus squalens. Bioresour Technol 97:2153-2159. doi:10.1016/j.biortech.2005.09.014

Eskizeybek V, Sarı F, Gülce H et al (2012) Preparation of the new polyaniline $/ \mathrm{ZnO}$ nanocomposite and its photocatalytic activity for degradation of methylene blue and malachite green dyes under UV and natural sun lights irradiations. Appl Catal B Environ 119-120:197-206. doi:10.1016/j.apcatb.2012.02.034

Farhadi K, Maleki R, Nezhad NM, Samadi N (2010) Spectrophotometric determination of malachite green residue in water samples after preconcentration on surfactant-coated alumina. Spectrosc Lett 43:101-107. doi:10.1080/00387010903278309

Forgacs E, Cserháti T, Oros G (2004) Removal of synthetic dyes from wastewaters: a review. Environ Int 30:953-971. doi:10.1016/j. envint.2004.02.001

Franca AS, Oliveira LS, Nunes AA (2010a) Malachite green adsorption by a residue-based microwave-activated adsorbent. CLEAN-Soil Air Water 38:843-849. doi:10.1002/clen. 201000153

Franca AS, Oliveira LS, Saldanha SA et al (2010b) Malachite green adsorption by mango (Mangifera indica L.) seed husks: kinetic, equilibrium and thermodynamic studies. Desalin Water Treat 19:241-248. doi:10.5004/dwt.2010.1105

Fu Y, Viraraghavan T (2001) Fungal decolorization of dye wastewaters: a review. Bioresour Technol 79:251-262. doi:10.1016/ S0960-8524(01)00028-1 
Gao J-F, Zhang Q, Wang J-H et al (2011) Contributions of functional groups and extracellular polymeric substances on the biosorption of dyes by aerobic granules. Bioresour Technol 102:805-813. doi:10.1016/j.biortech.2010.08.119

Garg VK, Gupta R, Bala Yadav A, Kumar R (2003) Dye removal from aqueous solution by adsorption on treated sawdust. Bioresour Technol 89:121-124. doi:10.1016/S09608524(03)00058-0

Garg VK, Kumar R, Gupta R (2004) Removal of malachite green dye from aqueous solution by adsorption using agro-industry waste: a case study of Prosopis cineraria. Dyes Pigments 62:1-10. doi:10.1016/j.dyepig.2003.10.016

Geng Z, Lin Y, Yu X et al (2012) Highly efficient dye adsorption and removal: a functional hybrid of reduced graphene oxide-Fe3O4 nanoparticles as an easily regenerative adsorbent. J Mater Chem 22:3527. doi:10.1039/c2jm15544c

Ghaedi M, Ansari A, Habibi MH, Asghari AR (2014) Removal of malachite green from aqueous solution by zinc oxide nanoparticle loaded on activated carbon: kinetics and isotherm study. J Ind Eng Chem 20:17-28. doi:10.1016/j.jiec.2013.04.031

Ghaedi M, Mosallanejad N (2014) Study of competitive adsorption of malachite green and sunset yellow dyes on cadmium hydroxide nanowires loaded on activated carbon. J Ind Eng Chem 20:1085-1096. doi:10.1016/j.jiec.2013.06.046

Ghaedi M, Shojaeipour E, Ghaedi AM, Sahraei R (2015) Isotherm and kinetics study of malachite green adsorption onto copper nanowires loaded on activated carbon: artificial neural network modeling and genetic algorithm optimization. Spectrochim Acta A Mol Biomol Spectrosc 142:135-149. doi:10.1016/j.saa.2015. 01.086

Godbole PT, Sawant AD (2006) Removal of malachite green from aqueous solutions using immobilised Saccharomyces cerevisiae. J Sci Ind Res 65:440-443

Gong R, Feng M, Zhao J et al (2009) Functionalization of sawdust with monosodium glutamate for enhancing its malachite green removal capacity. Bioresour Technol 100:975-978. doi:10.1016/ j.biortech.2008.06.031

Gong R, Jin Y, Chen F et al (2006) Enhanced malachite green removal from aqueous solution by citric acid modified rice straw. J Hazard Mater 137:865-870. doi:10.1016/j.jhazmat.2006.03. 010

Gong R, Sun J, Zhang D et al (2008) Kinetics and thermodynamics of basic dye sorption on phosphoric acid esterifying soybean hull with solid phase preparation technique. Bioresour Technol 99:4510-4514. doi:10.1016/j.biortech.2007.08.061

Gordon PF, Gregory P (1987) Organic Chemistry in Colour. Springer, Berlin, Heidelberg

Goursot A, Vasilyev V, Arbuznikov A (1997) Modeling of adsorption properties of zeolites: correlation with the structure. J Phys Chem B 101:6420-6428. doi:10.1021/jp971230b

Govindasamy V, Sahadevan R, Subramanian S, Mahendradas DK (2009) Removal of malachite green from aqueous solutions by perlite. Int J Chem React Eng 7:1-22. doi:10.2202/1542-6580.1889

Griffiths J (1976) Colour and constitution of organic molecules. Academic Press, London

Griffiths J (1981) Recent developments in the colour and constitution of organic dyes. Rev Prog Color Relat Top 11:37-57. doi:10. 1111/j.1478-4408.1981.tb03714.x

Guo Y, Yang S, Fu W et al (2003a) Adsorption of malachite green on micro- and mesoporous rice husk-based active carbon. Dyes Pigments 56:219-229. doi:10.1016/S0143-7208(02)00160-2

Guo Y, Zhang H, Tao N et al (2003b) Adsorption of malachite green and iodine on rice husk-based porous carbon. Mater Chem Phys 82:107-115. doi:10.1016/S0254-0584(03)00191-3

Gupta N, Kushwaha AK, Chattopadhyaya MC (2014) Application of potato (Solanum tuberosum) plant wastes for the removal of methylene blue and malachite green dye from aqueous solution. Arab J Chem. doi:10.1016/j.arabjc.2011.07.021

Gupta N, Kushwaha AK, Chattopadhyaya MC (2012) Adsorption studies of cationic dyes onto Ashoka (Saraca asoca) leaf powder. J Taiwan Inst Chem Eng 43:604-613. doi:10.1016/j. jtice.2012.01.008

Gupta VK, Jain R, Nayak A et al (2011) Removal of the hazardous dye - tartrazine by photodegradation on titanium dioxide surface. Mater Sci Eng C 31:1062-1067. doi:10.1016/j.msec.2011. 03.006

Gupta VK, Kumar R, Nayak A et al (2013) Adsorptive removal of dyes from aqueous solution onto carbon nanotubes: a review. Adv Colloid Interface Sci 193-194:24-34. doi:10.1016/j.cis. 2013.03.003

Gupta VK, Mittal A, Krishnan L, Gajbe V (2004) Adsorption kinetics and column operations for the removal and recovery of malachite green from wastewater using bottom ash. Sep Purif Technol 40:87-96. doi:10.1016/j.seppur.2004.01.008

Gupta VK, Nayak A (2012) Cadmium removal and recovery from aqueous solutions by novel adsorbents prepared from orange peel and $\mathrm{Fe}_{2} \mathrm{O}_{3}$ nanoparticles. Chem Eng J 180:81-90. doi:10. 1016/j.cej.2011.11.006

Gupta VK, Nayak A, Agarwal S (2015) Bioadsorbents for remediation of heavy metals: current status and their future prospects. Environ Eng Res 20:1-18

Gupta VK, Saleh TA (2013) Sorption of pollutants by porous carbon, carbon nanotubes and fullerene-an overview. Environ Sci Pollut Res 20:2828-2843. doi:10.1007/s11356-013-1524-1

Gupta VK, Srivastava SK, Mohan D (1997) Equilibrium uptake, sorption dynamics, process optimization, and column operations for the removal and recovery of malachite green from wastewater using activated carbon and activated slag. Ind Eng Chem Res 36:2207-2218. doi:10.1021/ie960442c

Gupta VK, Suhas (2009) Application of low-cost adsorbents for dye removal—a review. J Environ Manage 90:2313-2342. doi:10. 1016/j.jenvman.2008.11.017

Hamdaoui O, Chiha M, Naffrechoux E (2008a) Ultrasound-assisted removal of malachite green from aqueous solution by dead pine needles. Ultrason Sonochem 15:799-807. doi:10.1016/j. ultsonch.2008.01.003

Hamdaoui O, Saoudi F, Chiha M, Naffrechoux E (2008b) Sorption of malachite green by a novel sorbent, dead leaves of plane tree: equilibrium and kinetic modeling. Chem Eng J 143:73-84. doi:10.1016/j.cej.2007.12.018

Hameed BH, El-Khaiary MI (2008a) Equilibrium, kinetics and mechanism of malachite green adsorption on activated carbon prepared from bamboo by $\mathrm{K}_{2} \mathrm{CO}_{3}$ activation and subsequent gasification with CO2. J Hazard Mater 157:344-351. doi:10. 1016/j.jhazmat.2007.12.105

Hameed BH, El-Khaiary MI (2008b) Batch removal of malachite green from aqueous solutions by adsorption on oil palm trunk fibre: equilibrium isotherms and kinetic studies. J Hazard Mater 154:237-244. doi:10.1016/j.jhazmat.2007.10.017

Hameed BH, El-Khaiary MI (2008c) Kinetics and equilibrium studies of malachite green adsorption on rice straw-derived char. J Hazard Mater 153:701-708. doi:10.1016/j.jhazmat.2007.09.019

Hameed BH, El-Khaiary MI (2008d) Malachite green adsorption by rattan sawdust: isotherm, kinetic and mechanism modeling. J Hazard Mater 159:574-579. doi:10.1016/j.jhazmat.2008.02. 054

Hammud HH, Shmait A, Hourani N (2015) Removal of malachite green from water using hydrothermally carbonized pine needles. RSC Adv 5:7909-7920. doi:10.1039/C4RA15505J

Han R, Wang Y, Sun Q et al (2010) Malachite green adsorption onto natural zeolite and reuse by microwave irradiation. J Hazard Mater 175:1056-1061. doi:10.1016/j.jhazmat.2009.10.118 
Hasnat MA, Siddiquey IA, Saiful IS (2003) Photodegradation of malachite green in the aqueous medium. Indian J Chem Sect A 42:1865-1867

Hema M, Arivoli S (2007) Comparative study on the adsorption kinetics and thermodynamics of dyes onto acid activated low cost carbon. Int J Phys Sci 2:10-17

Heydartaemeh MR, Ardejani FD, Badii K et al (2014) $\mathrm{FeCl}_{2} / \mathrm{FeCl}_{3}$ perlite nanoparticles as a novel magnetic material for adsorption of green malachite dye. Arab J Sci Eng 39:3383-3392. doi:10. 1007/s13369-014-0978-x

Hu R, Huang J-B, Yang Z-P et al (2011) Biosorption of crystal violet and malachite green by Rhodotorula graminis Y-5. Ying Yong Sheng Tai Xue Bao J Appl Ecol Zhongguo Sheng Tai Xue Xue Hui Zhongguo Ke Xue Yuan Shenyang Ying Yong Sheng Tai Yan Jiu Suo Zhu Ban 22:3293-3299

Huang C-M, Chen L-C, Yang H-C et al (2012) Preparation of acrylic acid-modified chitin improved by an experimental design and its application in absorbing toxic organic compounds. J Hazard Mater 241-242:190-196. doi:10.1016/j.jhazmat.2012.09.032

Huang L, Weng X, Chen Z et al (2014) Green synthesis of iron nanoparticles by various tea extracts: comparative study of the reactivity. Spectrochim Acta A Mol Biomol Spectrosc 130:295-301. doi:10.1016/j.saa.2014.04.037

Huo S-H, Yan X-P (2012) Metal-organic framework MIL-100(Fe) for the adsorption of malachite green from aqueous solution. J Mater Chem 22:7449. doi:10.1039/c2jm16513a

Hussain F, Hojjati M, Okamoto M, Gorga RE (2006) Review article: polymer-matrix nanocomposites, processing, manufacturing, and application: an overview. J Compos Mater 40:1511-1575. doi:10.1177/0021998306067321

Idris MN, Ahmad ZA, Ahmad MA (2011) Adsorption equilibrium of malachite green dye onto rubber seed coat based activated carbon. Int J Basic Appl Sci 11:32-37

Iijima S (1991) Helical microtubules of graphitic carbon. Nature 354:56-58

Inbaraj BS, Sulochana N (2002) Basic dye adsorption on a low cost carbonaceous sorbent-kinetic and equilibrium studies. Indian J Chem Technol 9:201-208

Iqbal MJ, Ashiq MN (2007) Adsorption of dyes from aqueous solutions on activated charcoal. J Hazard Mater 139:57-66. doi:10.1016/j.jhazmat.2006.06.007

Issa AA, Al-Degs YS, Al-Ghouti MA, Olimat AAM (2014) Studying competitive sorption behavior of methylene blue and malachite green using multivariate calibration. Chem Eng J 240:554-564. doi:10.1016/j.cej.2013.10.084

Jalil AA, Triwahyono S, Yaakob MR et al (2012) Utilization of bivalve shell-treated Zea mays L. (maize) husk leaf as a low-cost biosorbent for enhanced adsorption of malachite green. Bioresour Technol 120:218-224. doi:10.1016/j.biortech.2012.06.066

Janoš P (2003) Sorption of basic dyes onto iron humate. Environ Sci Technol 37:5792-5798. doi:10.1021/es020142o

Janoš P, Michálek P, Turek L (2007) Sorption of ionic dyes onto untreated low-rank coal-oxihumolite: a kinetic study. Dyes Pigments 74:363-370. doi:10.1016/j.dyepig.2006.02.017

Janos P, Sedivý P, Rýznarová M, Grötschelová S (2005) Sorption of basic and acid dyes from aqueous solutions onto oxihumolite. Chemosphere 59:881-886. doi:10.1016/j.chemosphere.2004.11. 018

Janoš P, Šmídová V (2005) Effects of surfactants on the adsorptive removal of basic dyes from water using an organomineral sorbent-iron humate. J Colloid Interface Sci 291:19-27. doi:10. 1016/j.jcis.2005.04.065

Jasińska A, Bernat P, Paraszkiewicz K (2013) Malachite green removal from aqueous solution using the system rapeseed press cake and fungus Myrothecium roridum. Desalin Water Treat 51:7663-7671. doi:10.1080/19443994.2013.779939
Jayaraj R, Mohan MC, Prasath PMD, Khan TH (2011) Malachite green dye removal using the seaweed enteromorpha. J Chem 8:649-656. doi:10.1155/2011/141305

Jeon YS, Lei J, Kim J-H (2008) Dye adsorption characteristics of alginate/polyaspartate hydrogels. J Ind Eng Chem 14:726-731. doi:10.1016/j.jiec.2008.07.007

Jeyagowri B, Yamuna RT (2015) Potential efficacy of a mesoporous biosorbent Simarouba glauca seed shell powder for the removal of malachite green from aqueous solutions. Desalin Water Treat 57:11326-11336. doi:10.1080/19443994.2015.1042060

Ju Y, Yang S, Ding Y et al (2008) Microwave-assisted rapid photocatalytic degradation of malachite green in $\mathrm{TiO}_{2}$ suspensions: mechanism and pathways. $J$ Phys Chem A 112:11172-11177. doi:10.1021/jp804439z

Kadirvelu K, Kavipriya M, Karthika C et al (2003) Utilization of various agricultural wastes for activated carbon preparation and application for the removal of dyes and metal ions from aqueous solutions. Bioresour Technol 87:129-132. doi:10.1016/S09608524(02)00201-8

Kan Y, Yue Q, Kong J et al (2015) The application of activated carbon produced from waste printed circuit boards (PCBs) by $\mathrm{H} 3 \mathrm{PO} 4$ and steam activation for the removal of malachite green. Chem Eng J 260:541-549. doi:10.1016/j.cej.2014.09.047

Kaneva N, Stambolova I, Blaskov V et al (2010) Photocatalytic activity of nanostructured $\mathrm{ZnO}$ films prepared by two different methods for the photoinitiated decolorization of malachite green. J Alloys Compd 500:252-258. doi:10.1016/j.jallcom.2010.04. 020

Kannan C, Muthuraja K, Devi MR (2013) Hazardous dyes removal from aqueous solution over mesoporous aluminophosphate with textural porosity by adsorption. J Hazard Mater 244-245:10-20. doi:10.1016/j.jhazmat.2012.11.016

Kannan C, Sundaram T, Palvannan T (2008) Environmentally stable adsorbent of tetrahedral silica and non-tetrahedral alumina for removal and recovery of malachite green dye from aqueous solution. J Hazard Mater 157:137-145. doi:10.1016/j.jhazmat. 2007.12.116

Kannan RR, Rajasimman M, Rajamohan N, Sivaprakash B (2009) Brown marine algae Turbinaria conoides as biosorbent for malachite green removal: equilibrium and kinetic modeling. Front Environ Sci Eng China 4:116-122. doi:10.1007/s11783010-0006-7

Kant S, Pathania D, Singh P et al (2014) Removal of malachite green

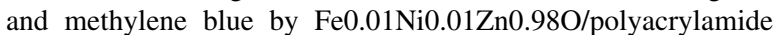
nanocomposite using coupled adsorption and photocatalysis. Appl Catal B Environ 147:340-352. doi:10.1016/j.apcatb.2013. 09.001

Karge HG, Weitkamp J (1989) Zeolites as catalysts, sorbents and detergent builders: applications and innovations. Elsevier, Amsterdam

Karimi A, Aghbolaghy M, Khataee A, Shoa Bargh S (2012) Use of enzymatic bio-fenton as a new approach in decolorization of malachite green. Sci World J. doi:10.1100/2012/691569

Kattri SD, Singh MK (1999) Adsorption of basic dyes from aqueous solution by natural adsorbent. Indian J Chem Technol 6:112-116

Khan AA, Ahmad R, Khan A, Mondal PK (2013) Preparation of unsaturated polyester $\mathrm{Ce}$ (IV) phosphate by plastic waste bottles and its application for removal of Malachite green dye from water samples. Arab J Chem 6:361-368. doi:10.1016/j.arabjc. 2010.10.012

Khan TA, Rahman R, Ali I et al (2014) Removal of malachite green from aqueous solution using waste pea shells as low-cost adsorbent-adsorption isotherms and dynamics. Toxicol Environ Chem 96:569-578. doi:10.1080/02772248.2014.969268

Khani H, Rofouei MK, Arab P et al (2010) Multi-walled carbon nanotubes-ionic liquid-carbon paste electrode as a super

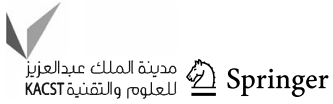


selectivity sensor: application to potentiometric monitoring of mercury ion(II). J Hazard Mater 183:402-409. doi:10.1016/j. jhazmat.2010.07.039

Khataee AR, Dehghan G, Zarei M et al (2011a) Neural network modeling of biotreatment of triphenylmethane dye solution by a green macroalgae. Chem Eng Res Des 89:172-178. doi:10.1016/ j.cherd.2010.05.009

Khataee AR, Zarei M, Dehghan G et al (2011b) Biotreatment of a triphenylmethane dye solution using a Xanthophyta alga: modeling of key factors by neural network. J Taiwan Inst Chem Eng 42:380-386. doi:10.1016/j.jtice.2010.08.006

Khattri S, Singh M (1999) Colour removal from dye wastewater using sugar cane dust as an adsorbent. Adsorpt Sci Technol 17:269-282

Khattri SD, Singh MK (2009) Removal of malachite green from dye wastewater using neem sawdust by adsorption. J Hazard Mater 167:1089-1094. doi:10.1016/j.jhazmat.2009.01.101

Khattri SD, Singh MK (2000) Colour removal from synthetic dye wastewater using a bioadsorbent. Water Air Soil Pollut 120:283-294. doi:10.1023/A:1005207803041

Kiani G, Dostali M, Rostami A, Khataee AR (2011) Adsorption studies on the removal of malachite green from aqueous solutions onto halloysite nanotubes. Appl Clay Sci 54:34-39. doi:10.1016/j.clay.2011.07.008

Kominami H, Kumamoto H, Kera Y, Ohtani B (2003) Photocatalytic decolorization and mineralization of malachite green in an aqueous suspension of titanium(IV) oxide nano-particles under aerated conditions: correlation between some physical properties and their photocatalytic activity. J Photochem Photobiol Chem 160:99-104. doi:10.1016/S1010-6030(03)00227-2

Krauskopf KB (1956) Factors controlling the concentrations of thirteen rare metals in sea-water. Geochim Cosmochim Acta 9:1B32. doi:10.1016/0016-7037(56)90055-2

Kuhar F, Papinutti L (2013) Protective effect of vanilloids against chemical stress on the white-rot fungus Ganoderma lucidum. J Environ Manage 124:1-7. doi:10.1016/j.jenvman.2013.03.040

Kumar KV (2006) Comparative analysis of linear and non-linear method of estimating the sorption isotherm parameters for malachite green onto activated carbon. J Hazard Mater 136:197-202. doi:10.1016/j.jhazmat.2005.09.018

Kumar KV (2007) Optimum sorption isotherm by linear and nonlinear methods for malachite green onto lemon peel. Dyes Pigments 74:595-597. doi:10.1016/j.dyepig.2006.03.026

Kumar KV, Porkodi K (2007) Batch adsorber design for different solution volume/adsorbent mass ratios using the experimental equilibrium data with fixed solution volume/adsorbent mass ratio of malachite green onto orange peel. Dyes Pigments 74:590-594. doi:10.1016/j.dyepig.2006.03.024

Kumar KV, Sivanesan S (2006) Pseudo second order kinetics and pseudo isotherms for malachite green onto activated carbon: comparison of linear and non-linear regression methods. J Hazard Mater 136:721-726. doi:10.1016/j.jhazmat.2006.01.003

Kumar KV, Sivanesan S, Ramamurthi V (2005) Adsorption of malachite green onto Pithophora sp., a fresh water algae: equilibrium and kinetic modelling. Process Biochem 40:2865-2872. doi:10.1016/j.procbio.2005.01.007

Kumar KY, Muralidhara HB, Nayaka YA et al (2013) Low-cost synthesis of metal oxide nanoparticles and their application in adsorption of commercial dye and heavy metal ion in aqueous solution. Powder Technol 246:125-136. doi:10.1016/j.powtec. 2013.05.017

Kurniawan A, Sutiono H, Indraswati N, Ismadji S (2012) Removal of basic dyes in binary system by adsorption using rarasaponinbentonite: revisited of extended Langmuir model. Chem Eng J 189-190:264-274. doi:10.1016/j.cej.2012.02.070
Kushwaha AK, Gupta N, Chattopadhyaya MC (2014) Removal of cationic methylene blue and malachite green dyes from aqueous solution by waste materials of Daucus carota. J Saudi Chem Soc 18:200-207. doi:10.1016/j.jscs.2011.06.011

Lata H, Gupta RK, Garg VK (2008) Removal of basic dye from aqueous solution using chemically modified parthenium hysterophorus linn. Biomass Chem Eng Commun 195:1185-1199. doi:10.1080/00986440701691103

Lee Y-C, Kim EJ, Yang J-W, Shin H-J (2011) Removal of malachite green by adsorption and precipitation using aminopropyl functionalized magnesium phyllosilicate. J Hazard Mater 192:62-70. doi:10.1016/j.jhazmat.2011.04.094

Lee Y-C, Kim J-Y, Shin H-J (2013) Removal of malachite green (MG) from aqueous solutions by adsorption, precipitation, and alkaline fading using talc. Sep Sci Technol 48:1093-1101. doi:10.1080/01496395.2012.723100

Levin L, Papinutti L, Forchiassin F (2004) Evaluation of Argentinean white rot fungi for their ability to produce lignin-modifying enzymes and decolorize industrial dyes. Bioresour Technol 94:169-176. doi:10.1016/j.biortech.2003.12.002

Li X, Li Y, Zhang S, Ye Z (2012) Preparation and characterization of new foam adsorbents of poly(vinyl alcohol)/chitosan composites and their removal for dye and heavy metal from aqueous solution. Chem Eng J 183:88-97. doi:10.1016/j.cej.2011.12.025

Lin K-YA, Chang H-A (2015) Ultra-high adsorption capacity of zeolitic imidazole framework-67 (ZIF-67) for removal of malachite green from water. Chemosphere. doi:10.1016/j. chemosphere.2015.01.041

Liu Y, Ohko Y, Zhang R et al (2010) Degradation of malachite green on $\mathrm{Pd} / \mathrm{WO}_{3}$ photocatalysts under simulated solar light. J Hazard Mater 184:386-391. doi:10.1016/j.jhazmat.2010.08.047

Low LW, Teng TT, Rafatullah M et al (2013) Adsorption studies of methylene blue and malachite green from aqueous solutions by pretreated lignocellulosic materials. Sep Sci Technol 48:1688-1698. doi:10.1080/01496395.2012.756912

Lv X, Xu Y, Lv K, Zhang G (2005) Photo-assisted degradation of anionic and cationic dyes over iron(III)-loaded resin in the presence of hydrogen peroxide. J Photochem Photobiol Chem 173:121-127. doi:10.1016/j.jphotochem.2005.01.011

Mahto TK, Chowdhuri AR, Sahu SK (2014) Polyaniline-functionalized magnetic nanoparticles for the removal of toxic dye from wastewater. J Appl Polym Sci 131:1-9. doi:10.1002/app.40840

Makeswari M, Santhi T (2013) Removal of malachite green dye from aqueous solutions onto microwave assisted zinc chloride chemical activated epicarp of Ricinus communis. J Water Resour Prot 5:222-238. doi:10.4236/jwarp.2013.52023

Malana MA, Ijaz S, Ashiq MN (2010) Removal of various dyes from aqueous media onto polymeric gels by adsorption process: their kinetics and thermodynamics. Desalination 263:249-257. doi:10. 1016/j.desal.2010.06.066

Malik R, Ramteke DS, Wate SR (2007) Adsorption of malachite green on groundnut shell waste based powdered activated carbon. Waste Manag 27:1129-1138. doi:10.1016/j.wasman. 2006.06.009

Mall ID, Srivastava VC, Agarwal NK, Mishra IM (2005) Adsorptive removal of malachite green dye from aqueous solution by bagasse fly ash and activated carbon-kinetic study and equilibrium isotherm analyses. Colloids Surf Physicochem Eng Asp 264:17-28. doi:10.1016/j.colsurfa.2005.03.027

Man LW, Kumar P, Teng TT, Wasewar KL (2012) Design of experiments for malachite green dye removal from wastewater using thermolysis - coagulation-flocculation. Desalin Water Treat 40:260-271. doi:10.1080/19443994.2012.671257

Mane VS, Babu PVV (2011) Studies on the adsorption of brilliant green dye from aqueous solution onto low-cost $\mathrm{NaOH}$ treated 
saw dust. Desalination 273:321-329. doi:10.1016/j.desal.2011. 01.049

Mansa RF, Sipaut CS, Rahman IA et al (2016) Preparation of glycine-modified silica nanoparticles for the adsorption of malachite green dye. J Porous Mater 23:35-46. doi:10.1007/ s10934-015-0053-3

Méndez A, Fernández F, Gascó G (2007) Removal of malachite green using carbon-based adsorbents. Desalination 206:147-153. doi:10.1016/j.desal.2006.03.564

Meng L, Zhang X, Tang Y et al (2015) Hierarchically porous siliconcarbon-nitrogen hybrid materials towards highly efficient and selective adsorption of organic dyes. Sci Rep 5:7910. doi:10. 1038/srep07910

Mirzajani R, Ahmadi S (2015) Melamine supported magnetic iron oxide nanoparticles (Fe3O4@Mel) for spectrophotometric determination of malachite green in water samples and fish tissues. J Ind Eng Chem 23:171-178. doi:10.1016/j.jiec.2014.08.011

Mishra RR, Chandran P, Khan SS (2014) Equilibrium and kinetic studies on adsorptive removal of malachite green by the citratestabilized magnetite nanoparticles. RSC Adv 4:51787-51793. doi:10.1039/C4RA07651F

Mittal A (2006) Adsorption kinetics of removal of a toxic dye, malachite green, from wastewater by using hen feathers. J Hazard Mater 133:196-202. doi:10.1016/j.jhazmat.2005.10. 017

Mittal A, Krishnan L, Gupta VK (2005) Removal and recovery of malachite green from wastewater using an agricultural waste material, de-oiled soya. Sep Purif Technol 43:125-133. doi:10. 1016/j.seppur.2004.10.010

Mittal A, Mittal J, Malviya A, Gupta VK (2009) Adsorptive removal of hazardous anionic dye "Congo Red" from wastewater using waste materials and recovery by desorption. J Colloid Interface Sci 340:16-26. doi:10.1016/j.jcis.2009.08.019

Modirshahla N, Behnajady MA (2006) Photooxidative degradation of malachite green (MG) by $\mathrm{UV} / \mathrm{H}_{2} \mathrm{O}_{2}$ : influence of operational parameters and kinetic modeling. Dyes Pigments 70:54-59. doi:10.1016/j.dyepig.2005.04.012

Mohammadi A, Daemi H, Barikani M (2014) Fast removal of malachite green dye using novel superparamagnetic sodium alginate-coated $\mathrm{Fe} 3 \mathrm{O} 4$ nanoparticles. Int $\mathrm{J}$ Biol Macromol 69:447-455. doi:10.1016/j.ijbiomac.2014.05.042

Mondal S (2008) Methods of dye removal from dye house effluentan overview. Environ Eng Sci 25:383-396. doi:10.1089/ees. 2007.0049

Moumeni O, Hamdaoui O (2012) Intensification of sonochemical degradation of malachite green by bromide ions. Ultrason Sonochem 19:404-409. doi:10.1016/j.ultsonch.2011.08.008

Nandi BK, Goswami A, Purkait MK (2009) Adsorption characteristics of brilliant green dye on kaolin. J Hazard Mater 161:387-395. doi:10.1016/j.jhazmat.2008.03.110

Nethaji S, Sivasamy A, Kumar RV, Mandal AB (2013) Preparation of char from lotus seed biomass and the exploration of its dye removal capacity through batch and column adsorption studies. Environ Sci Pollut Res Int 20:3670-3678. doi:10.1007/s11356012-1267-4

Nethaji S, Sivasamy A, Mandal AB (2012) Adsorption isotherms, kinetics and mechanism for the adsorption of cationic and anionic dyes onto carbonaceous particles prepared from Juglans regia shell biomass. Int J Environ Sci Technol 10:231-242. doi:10.1007/s13762-012-0112-0

Nethaji S, Sivasamy A, Thennarasu G, Saravanan S (2010) Adsorption of malachite green dye onto activated carbon derived from Borassus aethiopum flower biomass. J Hazard Mater 181:271-280. doi:10.1016/j.jhazmat.2010.05.008

$\mathrm{Ng}$ HW, Lee LY, Chan WL et al (2015) Luffa acutangula peel as an effective natural biosorbent for malachite green removal in aqueous media: equilibrium, kinetic and thermodynamic investigations. Desalin Water Treat 57:7302-7311. doi:10.1080/ 19443994.2015.1016460

Nidheesh PV, Gandhimathi R, Ramesh ST (2013) Degradation of dyes from aqueous solution by Fenton processes: a review. Environ Sci Pollut Res 20:2099-2132. doi:10.1007/s11356-012$1385-\mathrm{Z}$

Odom TW, Huang J-L, Kim P, Lieber CM (1998) Atomic structure and electronic properties of single-walled carbon nanotubes. Nature 391:62-64. doi:10.1038/34145

Oladoja NA, Aliu YD (2009) Snail shell as coagulant aid in the alum precipitation of malachite green from aqua system. J Hazard Mater 164:1496-1502. doi:10.1016/j.jhazmat.2008.09.114

Onal Y (2006) Kinetics of adsorption of dyes from aqueous solution using activated carbon prepared from waste apricot. J Hazard Mater 137:1719-1728. doi:10.1016/j.jhazmat.2006.05.036

Önal Y, Akmil-Başar C, Eren D et al (2006) Adsorption kinetics of malachite green onto activated carbon prepared from Tunçbilek lignite. J Hazard Mater 128:150-157. doi:10.1016/j.jhazmat. 2005.07.055

Önal Y, Akmil-Başar C, Sarıcı-Özdemir Ç (2007) Investigation kinetics mechanisms of adsorption malachite green onto activated carbon. J Hazard Mater 146:194-203. doi:10.1016/j. jhazmat.2006.12.006

Özdemir A, Keskin CS (2009) Removal of a binary dye mixture of congo red and malachite green from aqueous solutions using a bentonite adsorbent. Clays Clay Miner 57:695-705. doi:10.1346/ CCMN.2009.0570603

Ozdes D, Gundogdu A, Duran C, Senturk HB (2010) Evaluation of adsorption characteristics of malachite green onto Almond Shell (Prunus dulcis). Sep Sci Technol 45:2076-2085. doi:10.1080/ 01496395.2010.504479

Pan X, Zhang D (2009) Removal of malachite green from water by Firmiana simplex wood fiber. Electron J Biotechnol 12:9-10

Pandit P, Basu S (2004) Removal of ionic dyes from water by solvent extraction using reverse micelles. Environ Sci Technol 38:2435-2442

Papinutti L, Mouso N, Forchiassin F (2006) Removal and degradation of the fungicide dye malachite green from aqueous solution using the system wheat bran-Fomes sclerodermeus. Enzyme Microb Technol 39:848-853. doi:10.1016/j.enzmictec.2006.01. 013

Parida SK, Dash S, Patel S, Mishra BK (2006) Adsorption of organic molecules on silica surface. Adv Colloid Interface Sci 121:77-110. doi:10.1016/j.cis.2006.05.028

Patil MR, Shrivastava VS (2014) Adsorption of malachite green by polyaniline-nickel ferrite magnetic nanocomposite: an isotherm and kinetic study. Appl Nanosci 5:809-816. doi:10.1007/ s13204-014-0383-5

Paul M, Pal N, Bhaumik A (2012) Selective adsorption and release of cationic organic dye molecules on mesoporous borosilicates. Mater Sci Eng C Mater Biol Appl 32:1461-1468. doi:10.1016/j. msec.2012.04.026

Pearce CI, Lloyd JR, Guthrie JT (2003) The removal of colour from textile wastewater using whole bacterial cells: a review. Dyes Pigments 58:179-196. doi:10.1016/S0143-7208(03)00 064-0

Pehlivan E, Arslan G (2007) Removal of metal ions using lignite in aqueous solution-low cost biosorbents. Fuel Process Technol 88:99-106. doi:10.1016/j.fuproc.2006.09.004

Pirsaheb M, Shahmoradi B, Khosravi T et al (2015) Solar degradation of malachite green using nickel-doped $\mathrm{TiO}_{2}$ nanocatalysts. Desalin Water Treat 57:9881-9888. doi:10.1080/19443994. 2015.1033764

Podstawczyk D, Witek-Krowiak A, Chojnacka K, Sadowski Z (2014) Biosorption of malachite green by eggshells: mechanism

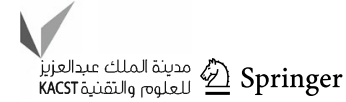


identification and process optimization. Bioresour Technol 160:161-165. doi:10.1016/j.biortech.2014.01.015

Porkodi K, Vasanth Kumar K (2007) Equilibrium, kinetics and mechanism modeling and simulation of basic and acid dyes sorption onto jute fiber carbon: eosin yellow, malachite green and crystal violet single component systems. J Hazard Mater 143:311-327. doi:10.1016/j.jhazmat.2006.09.029

Pourjavadi A, Doulabi M, Doroudian M (2014) Adsorption characteristics of malachite green dye onto novel kappa-carrageenan-gpolyacrylic acid/ $\mathrm{TiO}_{2}-\mathrm{NH}_{2}$ hydrogel nanocomposite. J Iran Chem Soc 11:1057-1065. doi:10.1007/s13738-013-0374-6

Pradeep Sekhar C, Kalidhasan S, Rajesh V, Rajesh N (2009) Biopolymer adsorbent for the removal of malachite green from aqueous solution. Chemosphere 77:842-847. doi:10.1016/j. chemosphere.2009.07.068

Pradhan AC, Parida KM, Nanda B (2011) Enhanced photocatalytic and adsorptive degradation of organic dyes by mesoporous $\mathrm{Cu} /$ $\mathrm{Al}_{2} \mathrm{O}_{3}-\mathrm{MCM}-41$ : intra-particle mesoporosity, electron transfer and $\mathrm{OH}$ radical generation under visible light. Dalton Trans Camb Engl 40:7348-7356. doi:10.1039/c1dt10363f

Prado AGS, Costa LL (2009) Photocatalytic decouloration of malachite green dye by application of $\mathrm{TiO} 2$ nanotubes. J Hazard Mater 169:297-301. doi:10.1016/j.jhazmat.2009.03. 076

Rahman IA, Saad B, Shaidan S, Sya Rizal ES (2005) Adsorption characteristics of malachite green on activated carbon derived from rice husks produced by chemical-thermal process. Bioresour Technol 96:1578-1583. doi:10.1016/j.biortech.2004.12.015

Rajabi HR, Khani O, Shamsipur M, Vatanpour V (2013) Highperformance pure and $\mathrm{Fe} 3+$-ion doped $\mathrm{ZnS}$ quantum dots as green nanophotocatalysts for the removal of malachite green under UV-light irradiation. J Hazard Mater 250-251:370-378. doi:10.1016/j.jhazmat.2013.02.007

Rajabi M, Mirza B, Mahanpoor K et al (2016) Adsorption of malachite green from aqueous solution by carboxylate group functionalized multi-walled carbon nanotubes: determination of equilibrium and kinetics parameters. $J$ Ind Eng Chem 34:130-138. doi:10.1016/j.jiec.2015.11.001

Rajesh Kannan R, Rajasimman M, Rajamohan N, Sivaprakash B (2010) Equilibrium and kinetic studies on sorption of malachite green using hydrilla verticillata biomass. Int $\mathrm{J}$ Environ Res $4: 817-824$

Rajeshkannan R, Rajamohan N, Rajasimman M (2009) Removal of malachite green from aqueous solution by sorption on hydrilla verticillata biomass using response surface methodology. Front Chem Eng China 3:146-154. doi:10.1007/s11705-009-0007-x

Rajeshkannan R, Rajasimman M, Rajamohan N (2011) Decolourization of malachite green using tamarind seed: optimization, isotherm and kinetic studies. Chem Ind Chem Eng Quart 17:67-79. doi:10.2298/CICEQ100716056R

Rajeshkannan R, Rajasimman M, Rajamohan N (2010) Removal of malachite green from aqueous solution using hydrilla verticillata-optimization, equilibrium and kinetic studies. Int $\mathrm{J}$ Civ Environ Eng 2:222-229

Rajeshwarisivaraj Sivakumar S, Senthilkumar P, Subburam V (2001) Carbon from Cassava peel, an agricultural waste, as an adsorbent in the removal of dyes and metal ions from aqueous solution. Bioresour Technol 80:233-235. doi:10.1016/S09608524(00)00179-6

Rajeshwarisivaraj, Subburam V (2002) Activated parthenium carbon as an adsorbent for the removal of dyes and heavy metal ions from aqueous solution. Bioresour Technol 85:205-206. doi:10. 1016/S0960-8524(02)00089-5

Rajgopal S, Karthikeyan T, Prakash Kumar BG, Miranda LR (2006) Utilization of fluidized bed reactor for the production of adsorbents in removal of malachite green. Chem Eng J 116:211-217. doi:10.1016/j.cej.2005.09.026

Ramaraju B, Manoj Kumar Reddy P, Subrahmanyam C (2014) Low cost adsorbents from agricultural waste for removal of dyes. Environ Prog Sustain Energy 33:38-46. doi:10.1002/ep.11742

Rauf MA, Bukallah SB, Hamour FA, Nasir AS (2008) Adsorption of dyes from aqueous solutions onto sand and their kinetic behavior. Chem Eng J 137:238-243. doi:10.1016/j.cej.2007.04. 025

Raval NP, Shah PU, Ladha DG et al (2016a) Comparative study of chitin and chitosan beads for the adsorption of hazardous anionic azo dye Congo Red from wastewater. Desalin Water Treat 57:9247-9262. doi:10.1080/19443994.2015.1027959

Raval NP, Shah PU, Shah NK (2016b) Nanoparticles loaded biopolymer as effective adsorbent for adsorptive removal of malachite green from aqueous solution. Water Conserv Sci Eng 1:69-81. doi:10.1007/s41101-016-0004-0

Raval NP, Shah PU, Shah NK (2016c) Adsorptive amputation of hazardous azo dye Congo red from wastewater: a critical review. Environ Sci Pollut Res 23:14810-14853. doi:10.1007/s11356016-6970-0

Raval NP, Shah PU, Shah NK (2016d) Adsorptive removal of nickel(II) ions from aqueous environment: a review. J Environ Manag 179:1-20. doi:10.1016/j.jenvman.2016.04.045

Ren S, Guo J, Zeng G, Sun G (2006) Decolorization of triphenylmethane, azo, and anthraquinone dyes by a newly isolated Aeromonas hydrophila strain. Appl Microbiol Biotechnol 72:1316-1321. doi:10.1007/s00253-006-0418-2

Robaina NF, Soriano S, Cassella RJ (2009) Polyurethane foam loaded with SDS for the adsorption of cationic dyes from aqueous medium: multivariate optimization of the loading process. J Hazard Mater 167:653-659. doi:10.1016/j.jhazmat.2009.01. 033

Robinson T, McMullan G, Marchant R, Nigam P (2001) Remediation of dyes in textile effluent: a critical review on current treatment technologies with a proposed alternative. Bioresour Technol 77:247-255. doi:10.1016/S0960-8524(00)00080-8

Rogaieh Z, Gharbani P (2013) Study on malachite green dye removal by adsorption onto activated nano-alumina. Appl Chem Environ 3:71-77

Rong X, Qiu F, Qin J et al (2014) Removal of malachite green from the contaminated water using a water-soluble melamine/maleic anhydride sorbent. J Ind Eng Chem 20:3808-3814. doi:10.1016/ j.jiec.2013.12.083

Roosta M, Ghaedi M, Shokri N et al (2014) Optimization of the combined ultrasonic assisted/adsorption method for the removal of malachite green by gold nanoparticles loaded on activated carbon: experimental design. Spectrochim Acta A Mol Biomol Spectrosc 118:55-65. doi:10.1016/j.saa.2013.08.082

Safarik I, Lunackova P, Mosiniewicz-Szablewska E et al (2007) Adsorption of water-soluble organic dyes on ferrofluid-modified sawdust. Holzforschung 61:247-253. doi:10.1515/HF.2007.060

Šafařík I, Šafaříková M, Vrchotová N (1995) Study of sorption of triphenylmethane dyes on a magnetic carrier bearing an immobilized copper phthalocyanine dye. Collect Czechoslov Chem Commun 60:34-42. doi:10.1135/cccc19950034

Šafařík I, Šafaříková M (2002) Detection of low concentrations of malachite green and crystal violet in water. Water Res 36:196-200. doi:10.1016/S0043-1354(01)00243-3

Saha P, Chowdhury S, Gupta S et al (2010a) Assessment on the removal of malachite green using tamarind fruit shell as biosorbent. CLEAN-Soil Air Water 38:437-445. doi:10.1002/ clen.200900234

Saha P, Chowdhury S, Gupta S, Kumar I (2010b) Insight into adsorption equilibrium, kinetics and thermodynamics of 
malachite green onto clayey soil of Indian origin. Chem Eng J 165:874-882. doi:10.1016/j.cej.2010.10.048

Saha S, Pal A (2014) Microporous assembly of $\mathrm{MnO}_{2}$ nanosheets for malachite green degradation. Sep Purif Technol 134:26-36. doi:10.1016/j.seppur.2014.07.021

Saha S, Wang JM, Pal A (2012) Nano silver impregnation on commercial $\mathrm{TiO} 2$ and a comparative photocatalytic account to degrade malachite green. Sep Purif Technol 89:147-159. doi:10. 1016/j.seppur.2012.01.012

Saleh TA, Gupta VK (2011) Functionalization of tungsten oxide into MWCNT and its application for sunlight-induced degradation of rhodamine B. J Colloid Interface Sci 362:337-344. doi:10.1016/ j.jcis.2011.06.081

Salima A, Benaouda B, Noureddine B, Duclaux L (2013) Application of Ulva lactuca and Systoceira stricta algae-based activated carbons to hazardous cationic dyes removal from industrial effluents. Water Res 47:3375-3388. doi:10.1016/j.watres.2013. 03.038

Salleh MAM, Mahmoud DK, Karim WAWA, Idris A (2011) Cationic and anionic dye adsorption by agricultural solid wastes: a comprehensive review. Desalination 280:1-13. doi:10.1016/j. desal.2011.07.019

Samiey B, Toosi AR (2010) Adsorption of malachite green on silica gel: effects of $\mathrm{NaCl}, \mathrm{pH}$ and 2-propanol. J Hazard Mater 184:739-745. doi:10.1016/j.jhazmat.2010.08.101

Sánchez PCM, Pariente JP (2011) Zeolites and ordered porous solids: fundamentals and applications. Editorial Universitat Politècnica de València, Valencia

Sand LB, Mumpton FA (1978) Natural zeolites: occurrence, properties, and use. Pergamon Press Inc, Elmsford

Santhi T, Manonmani S (2011) Malachite green removal from aqueous solution by the peel of cucumis sativa fruit. CLEANSoil Air Water 39:162-170. doi:10.1002/clen.201000077

Santhi T, Manonmani S, Smitha T (2010a) Kinetics and isotherm studies on cationic dyes adsorption onto annona squmosa seed activated carbon. Int J Eng Sci Technol 2:287-295

Santhi T, Manonmani S, Smitha T (2010b) Removal of malachite green from aqueous solution by activated carbon prepared from the epicarp of Ricinus communis by adsorption. J Hazard Mater 179:178-186. doi:10.1016/j.jhazmat.2010.02.076

Saravanan R, Gupta VK, Prakash T et al (2013) Synthesis, characterization and photocatalytic activity of novel $\mathrm{Hg}$ doped $\mathrm{ZnO}$ nanorods prepared by thermal decomposition method. J Mol Liq 178:88-93. doi:10.1016/j.molliq.2012.11.012

Saravanan R, Prakash T, Gupta VK, Stephen A (2014) Tailoring the electrical and dielectric properties of $\mathrm{ZnO}$ nanorods by substitution. J Mol Liq 193:160-165. doi:10.1016/j.molliq.2013.12. 029

Saravanan R, Sacari E, Gracia F et al (2016) Conducting PANI stimulated $\mathrm{ZnO}$ system for visible light photocatalytic degradation of coloured dyes. J Mol Liq 221:1029-1033. doi:10.1016/j. molliq.2016.06.074

Sarkar AK, Pal A, Ghorai S et al (2014) Efficient removal of malachite green dye using biodegradable graft copolymer derived from amylopectin and poly(acrylic acid). Carbohydr Polym 111:108-115. doi:10.1016/j.carbpol.2014.04.042

Sartape AS, Mandhare AM, Jadhav VV et al (2015) Removal of malachite green dye from aqueous solution with adsorption technique using Limonia acidissima (wood apple) shell as low cost adsorbent. Arab J Chem. doi:10.1016/j.arabjc.2013.12.019

Savva I, Marinica O, Papatryfonos CA et al (2015) Evaluation of electrospun polymer- $\mathrm{Fe}_{3} \mathrm{O}_{4}$ nanocomposite mats in malachite green adsorption. RSC Adv 5:16484-16496. doi:10.1039/ C4RA16938G

Sayılkan F, Asiltürk M, Tatar P et al (2007a) Photocatalytic performance of Sn-doped $\mathrm{TiO}_{2}$ nanostructured mono and double layer thin films for malachite green dye degradation under UV and vis-lights. J Hazard Mater 144:140-146. doi:10.1016/j. jhazmat.2006.10.011

Sayllkan F, Asiltürk M, Tatar P et al (2007b) Preparation of re-usable photocatalytic filter for degradation of malachite green dye under UV and vis-irradiation. J Hazard Mater 148:735-744. doi:10. 1016/j.jhazmat.2007.03.036

Saylkan F, Asiltürk M, Tatar P et al (2008) Photocatalytic performance of Sn-doped $\mathrm{TiO}_{2}$ nanostructured thin films for photocatalytic degradation of malachite green dye under UV and VIS-lights. Mater Res Bull 43:127-134. doi:10.1016/j. materresbull.2007.02.012

Schmidt D, Shah D, Giannelis EP (2002) New advances in polymer/ layered silicate nanocomposites. Curr Opin Solid State Mater Sci 6:205-212. doi:10.1016/S1359-0286(02)00049-9

Sharma P, Kaur H, Sharma M, Sahore V (2011) A review on applicability of naturally available adsorbents for the removal of hazardous dyes from aqueous waste. Environ Monit Assess 183:151-195. doi:10.1007/s10661-011-1914-0

Sharma YC (2011) Adsorption characteristics of a low-cost activated carbon for the reclamation of colored effluents containing malachite green. J Chem Eng Data 56:478-484. doi:10.1021/ je1008922

Sharma YC, Singh B, Uma (2009) Fast removal of malachite green by adsorption on rice husk activated carbon. Open Environ Pollut Toxicol J 1:74-78. doi:10.2174/1876397900901010074

Shedbalkar U, Jadhav JP (2011) Detoxification of malachite green and textile industrial effluent by Penicillium ochrochloron. Biotechnol Bioprocess Eng 16:196-204. doi:10.1007/s12257010-0069-0

Shi Q, Zhang J, Zhang C et al (2010) Preparation of activated carbon from cattail and its application for dyes removal. J Environ Sci China 22:91-97

Shirmardi M, Mahvi AH, Hashemzadeh B et al (2013) The adsorption of malachite green (MG) as a cationic dye onto functionalized multi walled carbon nanotubes. Korean $J$ Chem Eng 30:1603-1608. doi:10.1007/s11814-013-0080-1

Singh A, Manju Rani S, Bishnoi NR (2012) Malachite green dye decolorization on immobilized dead yeast cells employing sequential design of experiments. Ecol Eng 47:291-296. doi:10.1016/j.ecoleng.2012.07.001

Singh DK, Rastogi K (2004) Adsorptive removal of basic dyes from aqueous phase onto activated carbon of used tea leaves: a kinetic and thermodynamic study. J Environ Sci Eng 46:293-302

Sivashankar R, Sathya AB, Vasantharaj K, Sivasubramanian V (2014) Magnetic composite an environmental super adsorbent for dye sequestration-a review. Environ Nanotechnol Monit Manag 1-2:36-49. doi:10.1016/j.enmm.2014.06.001

Solís M, Solís A, Pérez HI et al (2012) Microbial decolouration of azo dyes: a review. Process Biochem 47:1723-1748. doi:10.1016/j. procbio.2012.08.014

Sonawane GH, Shrivastava VS (2009) Kinetics of decolourization of malachite green from aqueous medium by maize cob (Zea maize): an agricultural solid waste. Desalination 247:430-441. doi:10.1016/j.desal.2009.01.006

Sonawane SH, Chaudhari PL, Ghodke SA et al (2009) Ultrasound assisted synthesis of polyacrylic acid-nanoclay nanocomposite and its application in sonosorption studies of malachite green dye. Ultrason Sonochem 16:351-355. doi:10.1016/j.ultsonch. 2008.10.008

Srinivasan A, Viraraghavan T (2010) Decolorization of dye wastewaters by biosorbents: a review. J Environ Manag 91:1915-1929. doi:10.1016/j.jenvman.2010.05.003

Srivastava R, Rupainwar DC (2011) A comparative evaluation for adsorption of dye on Neem bark and Mango bark powder. Indian J Technol 18:67-75 
Stephen Inbaraj B, Chiu CP, Ho GH et al (2006) Removal of cationic dyes from aqueous solution using an anionic poly- $\gamma$-glutamic acid-based adsorbent. J Hazard Mater 137:226-234. doi:10. 1016/j.jhazmat.2006.01.057

Sun H, Yang H, Huang W, Zhang S (2015a) Immobilization of laccase in a sponge-like hydrogel for enhanced durability in enzymatic degradation of dye pollutants. J Colloid Interface Sci 450:353-360. doi:10.1016/j.jcis.2015.03.037

Sun L, Hu S, Sun H et al (2015b) Malachite green adsorption onto $\mathrm{Fe} 3 \mathrm{O} 4 @ \mathrm{SiO} 2-\mathrm{NH} 2$ : isotherms, kinetic and process optimization. RSC Adv 5:11837-11844. doi:10.1039/C4RA13402H

Sun X-F, Wang S-G, Liu X-W et al (2008) Biosorption of malachite green from aqueous solutions onto aerobic granules: kinetic and equilibrium studies. Bioresour Technol 99:3475-3483. doi:10. 1016/j.biortech.2007.07.055

Suresh T, Annadurai G (2013) Synthesis, characterization and photocatalytic degradation of malachite green dye using titanium dioxide nanoparticles. Int J Res Environ Sci Technol 3:71-77

Suwandi AC, Indraswati N, Ismadji S (2012) Adsorption of $\mathrm{N}$-methylated diaminotriphenilmethane dye (malachite green) on natural rarasaponin modified kaolin. Desalin Water Treat 41:342-355. doi:10.1080/19443994.2012.664738

Syed SP (2011) Study of the removal of malachite green from aqueous solution by using solid agricultural waste. Res J Chem Sci 1:1

Tahir H, Hammed U, Sultan M, Jahanzeb Q (2010) Batch adsorption technique for the removal of malachite green and fast green dyes by using montmorillonite clay as adsorbent. Afr J Biotechnol 9:8206-8214

Tahir H, Sultan M, Jahanzeb Q (2009) Remediation of azo dyes by using household used black tea as an adsorbent. Afr J Biotechnol. doi:10.4314/ajb.v8i15.61858

Tahir SS, Rauf N (2006) Removal of a cationic dye from aqueous solutions by adsorption onto bentonite clay. Chemosphere 63:1842-1848. doi:10.1016/j.chemosphere.2005.10.033

Tang H, Zhou W, Zhang L (2012) Adsorption isotherms and kinetics studies of malachite green on chitin hydrogels. J Hazard Mater 209-210:218-225. doi:10.1016/j.jhazmat.2012.01.010

Tang Y, Hu T, Zeng Y et al (2014) Effective adsorption of cationic dyes by lignin sulfonate polymer based on simple emulsion polymerization: isotherm and kinetic studies. RSC Adv 5:3757-3766. doi:10.1039/C4RA12229A

Tanthapanichakoon W, Ariyadejwanich P, Japthong P et al (2005) Adsorption-desorption characteristics of phenol and reactive dyes from aqueous solution on mesoporous activated carbon prepared from waste tires. Water Res 39:1347-1353. doi:10. 1016/j.watres.2004.12.044

Tavakol M, Azar PA, Tehrani MS, Ghaedi M (2015) Silver nanoparticles loaded on activated carbon as a novels adsorbent for the competitive removal of malachite green and methylene blue. Int J Life Sci 9:75-92. doi:10.3126/ijls.v9i2.12053

Tavlieva MP, Genieva SD, Georgieva VG, Vlaev LT (2013) Kinetic study of brilliant green adsorption from aqueous solution onto white rice husk ash. J Colloid Interface Sci 409:112-122. doi:10. 1016/j.jcis.2013.07.052

Tayade RJ, Surolia PK, Kulkarni RG, Jasra RV (2007) Photocatalytic degradation of dyes and organic contaminants in water using nanocrystalline anatase and rutile TiO2. Sci Technol Adv Mater 8:455-462. doi:10.1016/j.stam.2007.05.006

Tehrani-Bagha AR, Nikkar H, Mahmoodi NM et al (2011) The sorption of cationic dyes onto kaolin: kinetic, isotherm and thermodynamic studies. Desalination 266:274-280. doi:10.1016/ j.desal.2010.08.036

Tian Y, Liu P, Wang X, Lin H (2011) Adsorption of malachite green from aqueous solutions onto ordered mesoporous carbons. Chem Eng J 171:1263-1269. doi:10.1016/j.cej.2011.05.040
Tolia J, Chakraborty M, Murthy Z (2012) Photocatalytic degradation of malachite green dye using doped and undoped $\mathrm{ZnS}$ nanoparticles. Pol J Chem Technol 14:16-21. doi:10.2478/v10026-0120065-6

Tsai W-T, Chen H-R (2010) Removal of malachite green from aqueous solution using low-cost chlorella-based biomass. J Hazard Mater 175:844-849. doi:10.1016/j.jhazmat.2009.10. 087

Uma, Banerjee S, Sharma YC (2013) Equilibrium and kinetic studies for removal of malachite green from aqueous solution by a low cost activated carbon. J Ind Eng Chem 19:1099-1105. doi:10. 1016/j.jiec.2012.11.030

Vasanth Kumar K, Ramamurthi V, Sivanesan S (2006) Biosorption of malachite green, a cationic dye onto Pithophora sp., a fresh water algae. Dyes Pigments 69:102-107. doi:10.1016/j.dyepig.2005. 02.005

Vasanth Kumar K, Sivanesan S (2007) Isotherms for Malachite Green onto rubber wood (Hevea brasiliensis) sawdust: comparison of linear and non-linear methods. Dyes Pigments 72:124-129. doi:10.1016/j.dyepig.2005.07.021

Vasu AE (2008) Studies on the removal of rhodamine B and malachite green from aqueous solutions by activated carbon. J Chem 5:844-852. doi:10.1155/2008/271615

Vijayaraghavan K, Premkumar Y, Jegan J (2015) Malachite green and crystal violet biosorption onto coco-peat: characterization and removal studies. Desalin Water Treat 57:6423-6431. doi:10. 1080/19443994.2015.1011709

Volesky B (1990) Biosorption of heavy metals. CRC Press, Boca Raton

Wan Ngah WS, Ariff NFM, Hashim A, Hanafiah MAKM (2010) Malachite green adsorption onto chitosan coated bentonite beads: isotherms, kinetics and mechanism. CLEAN-Soil Air Water 38:394-400. doi:10.1002/clen.200900251

Wan Ngah WS, Teong LC, Hanafiah MAKM (2011) Adsorption of dyes and heavy metal ions by chitosan composites: a review. Carbohydr Polym 83:1446-1456. doi:10.1016/j.carbpol.2010.11. 004

Wang D, Liu L, Jiang X et al (2015) Adsorption and removal of malachite green from aqueous solution using magnetic $\beta$ cyclodextrin-graphene oxide nanocomposites as adsorbents. Colloids Surf Physicochem Eng Asp 466:166-173. doi:10. 1016/j.colsurfa.2014.11.021

Wang H, Yuan X, Zeng G et al (2014a) Removal of malachite green dye from wastewater by different organic acid-modified natural adsorbent: kinetics, equilibriums, mechanisms, practical application, and disposal of dye-loaded adsorbent. Environ Sci Pollut Res Int 21:11552-11564. doi:10.1007/s11356-014-3025-2

Wang H-Y, Gao H-W (2009) Preparation of calcium oxalatebromopyrogallol red inclusion sorbent and application to treatment of cationic dye and heavy metal wastewaters. Environ Sci Pollut Res Int 16:339-347. doi:10.1007/s11356-008-0070-8

Wang J, Zhao G, Li Y et al (2014b) One-step fabrication of functionalized magnetic adsorbents with large surface area and their adsorption for dye and heavy metal ions. Dalton Trans Camb Engl 43:11637-11645. doi:10.1039/c4dt00694a

Wang L, Zhang J, Zhao R et al (2010) Adsorption of basic dyes on activated carbon prepared from Polygonum orientale Linn: equilibrium, kinetic and thermodynamic studies. Desalination 254:68-74. doi:10.1016/j.desal.2009.12.012

Wang Q, Chen C, Zhao D et al (2008a) Change of adsorption modes of dyes on fluorinated $\mathrm{TiO}_{2}$ and its effect on photocatalytic degradation of dyes under visible irradiation. Langmuir ACS J Surf Colloids 24:7338-7345. doi:10.1021/la800313s

Wang S, Ariyanto E (2007) Competitive adsorption of malachite green and $\mathrm{Pb}$ ions on natural zeolite. J Colloid Interface Sci 314:25-31. doi:10.1016/j.jcis.2007.05.032 
Wang XS (2009) Invasive freshwater macrophyte alligator weed: novel adsorbent for removal of malachite green from aqueous solution. Water Air Soil Pollut 206:215-223. doi:10.1007/ s11270-009-0097-6

Wang XS, Zhou Y, Jiang Y, Sun C (2008b) The removal of basic dyes from aqueous solutions using agricultural by-products. J Hazard Mater 157:374-385. doi:10.1016/j.jhazmat.2008.01.004

Waring DR, Hallas G (eds) (1990) The chemistry and application of dyes. Springer US, Boston

Wei A, Liu B, Zhao H et al (2014) Synthesis and formation mechanism of flowerlike architectures assembled from ultrathin $\mathrm{NiO}$ nanoflakes and their adsorption to malachite green and acid red in water. Chem Eng J 239:141-148. doi:10.1016/j.cej.2013. 10.079

Weng X, Huang L, Chen Z et al (2013) Synthesis of iron-based nanoparticles by green tea extract and their degradation of malachite. Ind Crops Prod 51:342-347. doi:10.1016/j.indcrop. 2013.09.024

Witek-Krowiak A (2013) Biosorption of malachite green from aqueous solutions by pine sawdust: equilibrium, kinetics and the effect of process parameters. Desalin Water Treat 51:3284-3294. doi:10.1080/19443994.2012.749053

Witek-Krowiak A, Szafran RG, Modelski S, Dawiec A (2012) Removal of cationic dyes from aqueous solutions using microspherical particles of fly ash. Water Environ Res Res Publ Water Environ Fed 84:162-169

Wu J, Jung B-G, Kim K-S et al (2009) Isolation and characterization of Pseudomonas otitidis WL-13 and its capacity to decolorize triphenylmethane dyes. J Environ Sci China 21:960-964

Wu X, Wang Y, Liu J et al (2010) Study of malachite green adsorption onto natural zeolite in a fixed-bed column. Desalin Water Treat 20:228-233. doi:10.5004/dwt.2010.1212

Xing Y, Deng D (2009) Enhanced adsorption of malachite green by EDTAD-modified sugarcane bagasse. Sep Sci Technol 44:2117-2131. doi:10.1080/01496390902775588

Xing Y, Wang G (2009) Poly(methacrylic acid)-modified sugarcane bagasse for enhanced adsorption of cationic dye. Environ Technol 30:611-619. doi:10.1080/09593330902838098

Xu R, Jia M, Zhang Y, Li F (2012) Sorption of malachite green on vinyl-modified mesoporous poly(acrylic acid)/SiO2 composite nanofiber membranes. Microporous Mesoporous Mater 149:111-118. doi:10.1016/j.micromeso.2011.08.024

Yagub MT, Sen TK, Afroze S, Ang HM (2014) Dye and its removal from aqueous solution by adsorption: a review. Adv Colloid Interface Sci 209:172-184. doi:10.1016/j.cis.2014.04.002

Yang C-H, Shih M-C, Chiu H-C, Huang K-S (2014) Magnetic Pycnoporus sanguineus-loaded alginate composite beads for removing dye from aqueous solutions. Mol Basel Switz 19:8276-8288. doi:10.3390/molecules 19068276
Yang J, Chen C, Ji H et al (2005) Mechanism of $\mathrm{TiO}_{2}$-assisted photocatalytic degradation of dyes under visible irradiation: photoelectrocatalytic study by $\mathrm{TiO}_{2}$-film electrodes. J Phys Chem B 109:21900-21907. doi:10.1021/jp0540914

Yu J, Chi R, He Z, Qi Y (2011) Adsorption performances of cationic dyes from aqueous solution on pyromellitic dianhydride modified sugarcane bagasse. Sep Sci Technol 46:452-459. doi:10. 1080/01496395.2010.510125

Zeng Y, Zhao L, Wu W et al (2013) Enhanced adsorption of malachite green onto carbon nanotube/polyaniline composites. J Appl Polym Sci 127:2475-2482. doi:10.1002/app.37947

Zhang G, Yi L, Deng H, Sun P (2014a) Dyes adsorption using a synthetic carboxymethyl cellulose-acrylic acid adsorbent. J Environ Sci China 26:1203-1211. doi:10.1016/S10010742(13)60513-6

Zhang H, Tang Y, Liu X et al (2011) Improved adsorptive capacity of pine wood decayed by fungi Poria cocos for removal of malachite green from aqueous solutions. Desalination 274:97-104. doi:10.1016/j.desal.2011.01.077

Zhang J, Li Y, Zhang C, Jing Y (2008) Adsorption of malachite green from aqueous solution onto carbon prepared from Arundo donax root. J Hazard Mater 150:774-782. doi:10.1016/j.jhazmat.2007. 05.036

Zhang L, Zhang H, Guo W, Tian Y (2014b) Removal of malachite green and crystal violet cationic dyes from aqueous solution using activated sintering process red mud. Appl Clay Sci 93-94:85-93. doi:10.1016/j.clay.2014.03.004

Zhang X, Yu H, Yang $\mathrm{H}$ et al (2015) Graphene oxide caged in cellulose microbeads for removal of malachite green dye from aqueous solution. J Colloid Interface Sci 437:277-282. doi:10. 1016/j.jcis.2014.09.048

Zhang X, Zhang S, Pan B et al (2012) Simple fabrication of polymerbased Trametes versicolor laccase for decolorization of malachite green. Bioresour Technol 115:16-20. doi:10.1016/j. biortech.2011.11.063

Zhou X-J, Guo W-Q, Yang S-S et al (2013) Ultrasonic-assisted ozone oxidation process of triphenylmethane dye degradation: evidence for the promotion effects of ultrasonic on malachite green decolorization and degradation mechanism. Bioresour Technol 128:827-830. doi:10.1016/j.biortech.2012.10.086

Zhou Y, Min Y, Qiao H et al (2015) Improved removal of malachite green from aqueous solution using chemically modified cellulose by anhydride. Int J Biol Macromol 74:271-277. doi:10.1016/j. ijbiomac.2014.12.020

Zou W, Gao S, Zou X, Bai H (2013) Adsorption of neutral red and malachite green onto grapefruit peel in single and binary systems. Water Environ Res Res Publ Water Environ Fed $85: 466-477$ 